A Procedure for Beamline Characterization and Tuning in Open-Ended Beamlines

A Dissertation
Presented to
the faculty of the School of Engineering and Applied Science
University of Virginia
in partial fulfillment
of the requirements for the degree
Doctor of Philosophy
by

Ryan Michael Bodenstein

December

2012 


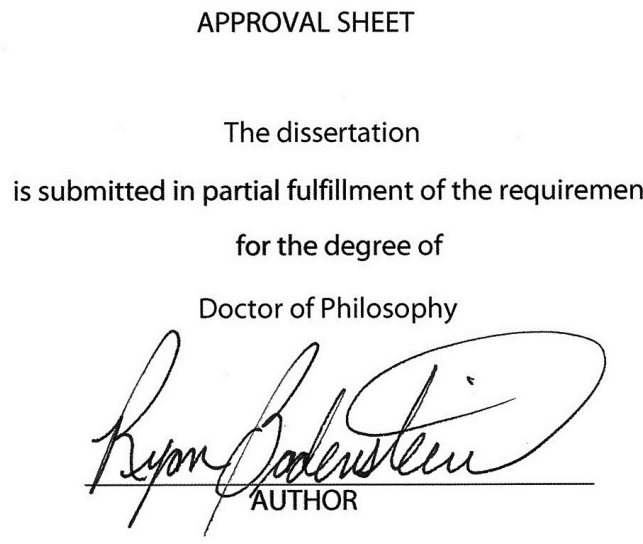

The dissertation has been read and approved by the examining committee:

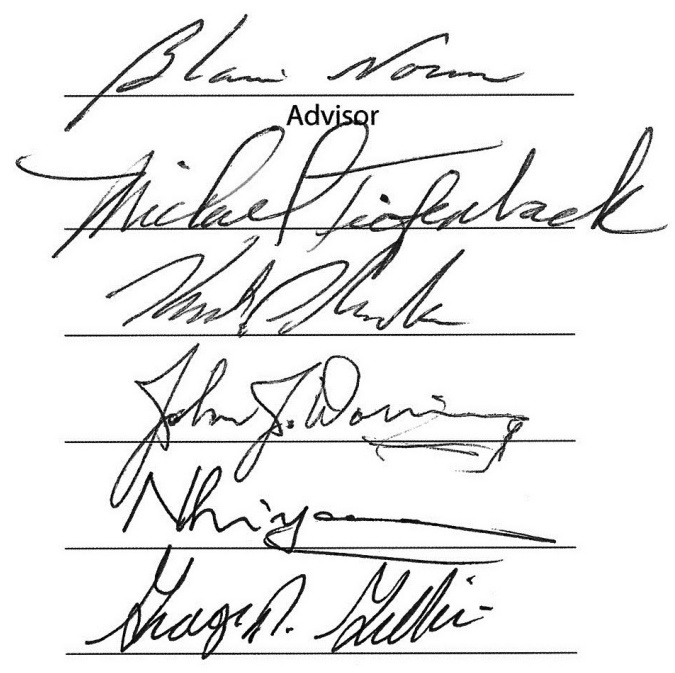

Accepted for the School of Engineering and Applied Science:

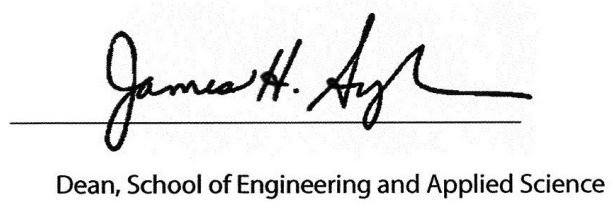

December

2012 


\title{
A Procedure for Beamline Characterization and Tuning in Open-Ended Beamlines
}

\author{
Ryan M. Bodenstein
}

11 December 2012 


\begin{abstract}
Standard closed-orbit techniques for Twiss parameter measurement are not applicable to the open-ended Continuous Electron Beam Accelerator Facility (CEBAF) at Jefferson Lab. The evolution of selected sets of real orbits in the accelerator models the behavior of a "synthetic" beam. This process should provide the distributed optical information needed to optimize beamline tuning for an open-ended system. This work will discuss the development and current state of this technique, as well as examples of its use in the CEBAF machine.
\end{abstract}




\section{Acknowledgments}

Throughout the process of writing this dissertation, I was frequently told that writing the acknowledgments section would be the easiest part. After all, I did the work necessary to earn my Ph.D. While it may be true that I did the necessary work, I cannot pretend that I was alone in this endeavor. Throughout the past eight and a half years, I have been surrounded by many people who

have helped me in some way. Sometimes this help was technical or instructive in nature, while other times it was personal or emotional. There have been so many people that have touched my life that I find it nearly impossible to remember them all. I am going to thank many of the people that have helped me earn this degree, but this list is not exhaustive. In fact, it is quite abridged. If you have helped me in any way to earn this degree, I thank you. If you do not see yourself listed in this section, please realize that it does not mean your contribution to my success is insignificant. It simply means that, given the time constraints placed upon me to submit this final work, I do not have time to name everybody.

I want to begin by thanking those that contributed to my pre-graduate school education. First and foremost, I want to thank my first Physics teacher, Tony Wayne. Mr. Wayne challenged me in a way that I had never been challenged, and I loved it. I fell in love with physics while a Junior at Albemarle High School, and it is all his fault. I ended up taking his AP class in my senior year, and was the only person to actually take the AP Physics exam that year. After his classes, I knew that I wanted to do physics for the rest of my life. Thank you for the inspiration, Mr. Wayne. Without your influence, I would not be where I am today.

At Mary Washington, I had a support network that may not exist at other schools. Dr. Bülent Atalay took an early interest in me, encouraging me and helping me to excel early in my undergraduate career. He was always available to help me in my struggles, both personal and academic. I remember spending many hours in his office discussing all sorts of topics. I also remember him supporting me after the loss of my father before my matriculation at Mary Washington. And who could forget the yearly Christmas parties at his home, or the trip to Princeton?

Dr. George King, III became my undergraduate advisor when I began showing interest in experimental physics. He challenged me in new ways, but was always more than supportive, often going far above and beyond what one would 
expect a professor to go in assisting a student. I spent many hours in his office, often frustrated at my inability to grasp concepts. He never once lost patience with me, which is a feat in itself. I grew to love experimental physics under his guidance.

Dr. Susan Matts began teaching at Mary Washington while I was a student there, and I took the first Classical Mechanics class that she offered. It challenged me in a way that I had not yet been challenged, and while I struggled with her methods at the time, I can now say that this challenge helped me grow as both a physicist and a student. I look back and know that the long takehome exams that encouraged independent thought prepared me for the years of graduate school that were to come.

Had it not been for these three professors, I would probably have lost my interest in physics. Rather than simply being an academic department, Mary Washington's Physics Department was more like a family. This is completely due to these three professors.

Next, I would like to thank those that helped me at The University of Virginia, especially those in the Engineering Physics Department and the Physics Department. Upon admittance to the graduate program at UVA, I entered a new stage in my life. The transition to graduate life was not an easy one for me, and there are many people who helped me make it through. All of my fellow students in the Physics and Engineering Physics departments that spent late nights on problem sets and studying for exams with me come to mind first. We used each other as sounding boards not only academically, but in our personal lives. We ate meals together, went to the gym together, and laughed together. We spent many late nights helping each other get through those first couple of years, and without this interaction, I do not know if I could have made it through.

Some of the staff and faculty in these programs also helped me greatly. Petra Reinke and William Soffa supported my efforts from the moment they became involved with the EP program. Professor Reinke went the extra mile to support me in my times of need, and I cannot express my appreciation of this. Jerry Floro later replaced Professor Soffa as co-chair of the EP program, and provided me with even more support. Professors Reinke and Floro supported me in my need for extra time in my research, acting as my voice to the Graduate Studies Committee. Without this support, I would not be where I am now. Thank you.

Vickie Thomas, Tammie Shifflett, Dawn Shifflett, Susan Bagby, and most recently Kim Fitzhugh-Higgins have been some of the most supportive administrative staff a person could ever hope for. I would be lost in paperwork and requirements without you all.

I must thank my Advisory Committee as well. You have all provided me the extra motivation needed to finish my work, provided great insights into my work, and went out of your ways to make yourselves available to me. Thank you.

To my advisor, Blaine Norum, I must acknowledge your guidance, knowledge, and patience. You limited the amount of stress that I had to deal with as a graduate student. I knew that I never had to worry about finding my own 
funding or dealing with other bureaucratic concerns. Additionally, your sense of humor and our often off-topic conversations were a great reprieve from the daily grind.

I will next turn my attention to those that helped me at Jefferson Lab. The CASA Group has been my home for the past six-plus years. I feel a bit like a family member in this group, having spent so much time here. The support structure has been excellent; both challenging and encouraging. Geoff Krafft brought me into this group after a discussion at UVA when I was taking his class. I could not be more appreciative. If it weren't for him, I would never have been able to become a member of such a diverse, brilliant group of people. At some point I think that every member of this group has helped me in some way. Alicia Hofler has provided me more help than I can even quantify, both professionally and personally. Todd Satogata, who has influenced me more than I can adequately express, has provided me with so much assistance that I cannot even begin to recount it here. His friendship and support helped to make this work possible. Yves Roblin, beyond beginning work on rayTrace with Michael Tiefenback, has been a constant source of insights that I may have otherwise never had.

Beyond the CASA group, I have had much support at Jefferson Lab. Michele Joyce provided me with so much programming support over the past few years that I cannot even begin to thank her. I keep promising her beers, but at this point I think I owe her a brewery. The operations group has always been supportive of my needs, always making sure to find a way to fit my research into their busy schedules. Additionally, the administrative staff at Jefferson Lab has always been pleasant and helpful.

I must also thank Michael Tiefenback, my supervisor and the man most responsible for helping me in my research. Beyond his normal advisory, supervisory, and administrative duties, Michael has also provided me with friendship and a personal level of support that is rare among supervisors. Our discussions would often devolve into off-topic discussions, but I always looked forward to these interactions. I must admit that it took me a while to get used to the way Michael communicated, and that I was often frustrated or confused. However, once I learned to understand Michael, I gained more insight from him than perhaps anyone else in my career to this point. His desire for me to not just learn the basics of a topic, but to gain mastery of it, has shaped me into the scientist I am today. His influence and our friendship will be lasting, and I cannot fully express my appreciation.

I must list separately one man who helped me through a very hard time in my life: Tim Latsko. He taught me how to deal with anxiety and stress, and helped me to get through one of the roughest times in my adult life. Thank you.

I have made so many friends over the past few years, and I cannot list them all here. They have all helped me in some manner; some personal, some professional, and some both. Ilkyoung Shin has been a great friend over the past few years, providing both professional input and a close friendship that is priceless. Paul Mattione, despite repeatedly destroying me in chess (except 
that once!), has always supported me through friendship and advice. Daniel Bowring, my sometimes gym partner and always good friend, not only provided support through his friendship but also through advice and shared experiences in the EP program. Vasiliy Morozov, beyond helping me learn Mad-X, has been a constant source of friendship and humor. Thank you, to all of my friends.

I also have to mention Adam Marshall, who has been one of my closest friends since we were forced to live together our Sophomore year of college. He has been a constant source of insight, humor, stress-relief, and of course friendship. Thanks, Adam.

I have also had the benefit of an amazing family, both immediate and extended. To the Valentines, who have been my extended family since my childhood, thank you for the years of love and support. To my uncle, Bobby Valentine, your love and support over the years cannot be measured. Between buying my textbooks through my undergraduate years, and being an inspirational figure in my life, I cannot fully express my gratitude. Thank you.

To the German family household, thank you for taking me in. You have been a second family to me, and without your support this journey would have been much more difficult. John German, Kathy German, Megan German, and Miranda Guzman, your love and support has helped me carry on with this work, even when I was less than agreeable to coexist with. Thank you.

To my extended family, thank you. Pat, you have always supported me as though I was your own child. Your love has always been a constant reminder of what patience and compassion can bring.

To my immediate family, words cannot express my love for you. Even when you do not understand what I am doing, or what I am going through, you have never wavered in your support of me. Tim, I am so happy that you are part of my family. Jyl, you are the best little sister that a person could ever ask for. I am so proud of you and all that you have accomplished. You are an inspiration to me. Jaeshaun, you are one of my favorite people in this world. You will always be Chewy to me, and I will spoil you for the rest of your life. Mom, thank you. Thank you for everything. Look at what you accomplished! You and John raised me right. If it weren't for you, who knows what I would be. As much as I have accomplished, it is nothing compared to what you have done. I love all of you. Thank you.

Finally, Marissa. I love you with ever fiber of my being. For the last two and a half years, you have been the brightest part of my life. Every time I hear your voice, every time I see your face, the problems of my day disappear. You have held me together for the past few years. You have kept me sane. I cannot thank you enough. Here's to the rest of our lives! I love you. 
To my family, and my father, John Valentine.

You are always in my thoughts. 


\section{Contents}

$\begin{array}{lll}\text { I Background } & 1\end{array}$

1 Outline of Jefferson Lab's CEBAF 3

1.1 Overview $[16,17,24] \ldots \ldots \ldots \ldots \ldots \ldots$

1.2 Magnetic Control of Beam Optics . . . . . . . . . . . . 5

1.2.1 Defining Magnetic Fields . . . . . . . . . . . . 5

1.2 .2 Dipole Magnets . . . . . . . . . . . . . . . . 7

1.2.3 Quadrupole Magnets . . . . . . . . . . . . . . . . . . 10

1.3 Beam Position Monitors $[9,19] \ldots \ldots \ldots$

2 Accelerator Physics Background 16

2.1 Beam Dynamics . . . . . . . . . . . . . . . . . 16

2.1 .1 Phase Space . . . . . . . . . . . . . 16

2.1.2 Matrix Formalism $[15,25,26,27] \ldots \ldots \ldots$

2.1.3 The Twiss Parameters $[27] \ldots \ldots \ldots \ldots$

3 Diagnostic Tools Currently in Use 22

3.1 Quadrupole Scan . . . . . . . . . . . . . . . . . . 22

3.2 The Courant-Snyder Method . . . . . . . . . . . . . . 24

4 A New Tool: rayTrace $\quad 26$

4.1 What is rayTrace? . . . . . . . . . . . . . 26

4.2 How rayTrace Works . . . . . . . . . . . . . . . 27

4.2 .1 Data Acquisition . . . . . . . . . . . . . . 27

4.2.2 Model Twiss Parameter Acquisition . . . . . . . . . 28

4.2 .3 Analysis of Data: fitphase . . . . . . . . . . . . . 28

4.2 .4 Tuning the Machine . . . . . . . . . . . . . . 29

4.3 rayTrace in Action: An Example of Use . . . . . . . . . . 30

\section{Noise Reduction and Uncertainty Analysis 33}

5 Singular Value Decomposition Analysis $\quad 34$

5.1 Least Squares and Singular Value Decomposition . . . . . . . . . 34

5.1 .1 Least Squares . . . . . . . . . . . . . . . . . . 34 
5.1.2 Singular Value Decomposition $[1,23,28] \ldots \ldots$. . . . . 35

$5.2 \quad$ SVD Noise Reduction $[18,23,28,29] \ldots \ldots \ldots$

5.2 .1 Raw rayTrace Data . . . . . . . . . . . . . . 39

$5.2 .2 \quad$ SVD Procedure . . . . . . . . . . . . . . . 41

III Resolution Test 52

6 Resolution Test 53

6.1 Background of Resolution Test . . . . . . . . . . . . . 53

6.2 Experimental Description and Setup . . . . . . . . . . 54

6.3 Analysis of Resolution Test Data . . . . . . . . . . . 55

6.3.1 Initial Analysis . . . . . . . . . . . . . . . . 55

6.3.2 Noise-reduced Analysis . . . . . . . . . . . . . . . . . 58

6.3.2.1 Comparing Raw and Noise-Reduced Data . . . . 58

6.3.3 Localizing A Known Optics Change . . . . . . . . . . . 61

6.3.4 Resolving the Quadrupole Change . . . . . . . . . . . . 64

6.3.4.1 Mathematical Review . . . . . . . . . . . . 64

6.3.4.2 Fitting With Mad-X . . . . . . . . . . 66

6.3.5 Uncertainty Analysis . . . . . . . . . . . . . . . . 69

6.3 .6 Interpretations . . . . . . . . . . . . . . . . 73

6.3.7 Conclusions of Resolution Test . . . . . . . . . . . 74

IV Summary and Conclusion $\quad 76$

$\mathbf{7}$ Conclusions $\quad \mathbf{7 7}$

7.1 Summary . . . . . . . . . . . . . . . . 77

7.2 Suggestions for Future Work . . . . . . . . . . . 78

\section{Appendices $\quad 79$}

A Additional Background Development $\quad \mathbf{8 0}$

A.1 Some Basic Physics . . . . . . . . . . . . . . . . . . . 80

A.1.1 Lagrangian Formulation . . . . . . . . . . . . . . 80

A.1.2 Hamiltonian Formulation . . . . . . . . . . . . . 82

A.2 Symplecticity . . . . . . . . . . . . . 83

B Scripts $\quad \mathbf{8 6}$

B.1 Example Lattice File and Mad-X Script . . . . . . . . . . . . 86

B.1.1 Lattice File . . . . . . . . . . . . . . . . . 86

B.1.2 Mad-X Script . . . . . . . . . . . . 86 


\section{List of Figures}

1.1 Diagram of CEBAF Layout . . . . . . . . . . . . . 3

1.2 West Arc . . . . . . . . . . . . . . . . 4

1.3 Beginning of the Recombiner and a Recombiner Dipole . . . . . 5

1.4 Corrector Dipole, BPM, Quadrupole in between Cryomodules . . 8

1.5 Schematic of Dipole [19] . . . . . . . . . . . . . . . . 9

1.6 C-type Dipole with Integration Path [19] . . . . . . . . . . . 10

1.7 Schematic of Quadrupole [19] . . . . . . . . . . . . . . . 11

1.8 Integration Path of Quadrupole $[19] \ldots \ldots \ldots \ldots$

1.9 Beam Position Monitor (BPM) . . . . . . . . . . . . . 12

1.10 Schematic of BPM used at CEBAF [19] . . . . . . . . 14

2.1 Standard Phase Space Ellipse Labeled with Twiss Parameters . . 20

3.1 Beam Radius Hyperbola and Phase Ellipse . . . . . . . . . . 23

3.2 Courant-Snyder Plots . . . . . . . . . . . . . . 25

3.3 Courant-Snyder Degeneracy . . . . . . . . . . . . . 25

4.1 The rayTrace GUI . . . . . . . . . . . . . . . 28

4.2 Overlaid Trajectories for 3 Pass CEBAF Machine . . . . . . . . 29

4.3 Injection Conditions in ARC $1 \ldots \ldots \ldots$

4.4 Y-Plane Aberration in Compton . . . . . . . . . . . 31

5.1 SVD Flow Chart $\ldots \ldots \ldots \ldots \ldots \ldots$

5.2 Format of Collected BPM Data . . . . . . . . . . . . 40

5.3 Raw X-Plane Data for ARC1 . . . . . . . . . . . . . . . 42

5.4 Detrended Raw X-Plane Data for ARC1 . . . . . . . . . . . 43

5.5 Singular Values for Non-Detrended Data: Arc1, X-Plane, X-Kick.

Axes are Singular Value (0-180) vs. Singular Value Index (0-50) . 44

5.6 Singular Values for Detrended Data: Arc 1, X-Plane, X-Kick.

Axes are Singular Value (0-180) vs. Singular Value Index (0-50) . 44

5.7 First 16 Temporal Basis Vectors for Arc 1, X-Plane, X-Kick . . . 45

5.8 First 16 Spatial Basis Vectors for Arc 1, X-Plane, X-Kick . . . 47

5.9 Spatial Basis Vectors, Temporal Basis Vectors, and Periodogram (FFT) of Temporal Basis Vectors . . . . . . . . . . . . . . 48

5.10 Detrended Raw Data For All BPMs in ARC1 . . . . . . . . . 49 
5.11 Detrended, Noise-Reduced Data For All BPMs in ARC1 . . . . 50

5.12 Detrended Raw vs. Noise-Reduced BPM \#29 (IPM1A23) in ARC1, X-Plane . . . . . . . . . . . . . . 51

$6.1 \beta$ Through ARC1 and LINAC2: Comparing Data and Model . . 55

6.2 Emittance $(\epsilon)$ Through ARC1 and LINAC2: Full Scale . . . . . 56

6.3 Emittance $(\epsilon)$ Through ARC1 and LINAC2: Zoomed In . . . . 56

6.4 Phase Ellipse From Raw Data . . . . . . . . . . . . . . . . 57

6.5 Comparison of Raw and Noise-Reduced $\beta$ From Baseline Data . 58

6.6 Comparison of Raw and Noise-Reduced $\epsilon$ From Baseline Data: Full Scale . . . . . . . . . . . . . . . . . . 59

6.7 Comparison of Raw and Noise-Reduced $\epsilon$ From Baseline Data:

Zoomed In . . . . . . . . . . . . . . . . . . . . . . . . 59

6.8 Comparison of BPM Phase Ellipse Before and After Noise Reduction .................... 60

6.9 Comparison of Baseline $\epsilon \ldots \ldots \ldots$. . . . . . . . . 61

6.10 Comparison of Baseline $\beta \ldots \ldots \ldots$. . . . . . . . 62

6.11 Overlaid $\beta$ Through ARC1 and LINAC2 . . . . . . . . . 63

6.12 Overlaid $\epsilon$ Through ARC1 and LINAC2 . . . . . . . . . . 63

6.13 Overlaid $\beta$ At End of ARC1 Through LINAC2 $\ldots \ldots \ldots . . .64$

6.14 RMS Beam Size For X-Plane (left) and Y-Plane (right) . . . . 72 


\section{Part I}

\section{Background}




\section{Introduction}

The Continuous Electron Beam Accelerator Facility (CEBAF) at Jefferson Lab is a medium-energy electron accelerator consisting of two superconducting linacs connected by independent recirculation arcs. It can accurately be described as a series of concatenated transfer lines. Polarized electrons pass through the racetrack system up to five times, reaching a maximum energy of $6 \mathrm{GeV}$, and can then be used by up to three experimental halls. Beam quality requirements include specifications for beam size and position stability at various locations, including the physics target in the experimental hall. The accelerator is in the process of having its energy doubled and a fourth experimental hall added. With this upgrade, the beam must be more tightly controlled in order to minimize the emittance growth.

Beam matching requirements internal to the accelerator are expected to be more stringent after the ongoing $12 \mathrm{GeV}$ Upgrade is completed. Current methods of measuring the optical parameters of the beam are able to provide local information, but fail to provide a global understanding of the optics of the machine. The diagnostics in use do not provide adequate measurements of the evolution of the Twiss parameters through the accelerator, so optical errors cannot easily be localized.

The method we are developing uses a family of differential orbits selected as a surrogate for a real beam: a "synthetic beam" if you will. The goals of this method are the identification of point errors and detection of distributed errors along the beamline. By injecting into the beamline a family of rays which occupies the phase space boundary of the design beam, the transformed Twiss parameters can be read off from local beam position measurements. The horizontal $(\mathrm{X})$ and vertical $(\mathrm{Y})$ trajectories are measured simultaneously at every beam position monitor (BPM) in the machine. The extreme values of the $\mathrm{X}$ and $\mathrm{Y}$ measurements for the injected rays provide a model-independent measure of the envelope of the "synthetic beam". Short-range optical modeling provides the necessary angular information from beam position measurements. The bunch charge and self-field effects of the CEBAF beam are small enough that the transfer properties of the lattice in the zero-current limit are applicable for use in characterizing the beam optics. 


\section{Chapter 1}

\section{Outline of Jefferson Lab's CEBAF}

\subsection{Overview $[16,17,24]$}

Jefferson Lab's Continuous Electron Beam Accelerator Facility (CEBAF) was originally constructed as a $4 \mathrm{GeV}, 200 \mu \mathrm{A}$ recirculating electron accelerator. The beam energy was later upgraded to $6 \mathrm{GeV}$, and the machine is currently undergoing a further upgrade to reach energies of up to $12 \mathrm{GeV}$. Figure 1.1 shows a general schematic of CEBAF, including the placement of the new experimental Hall D.

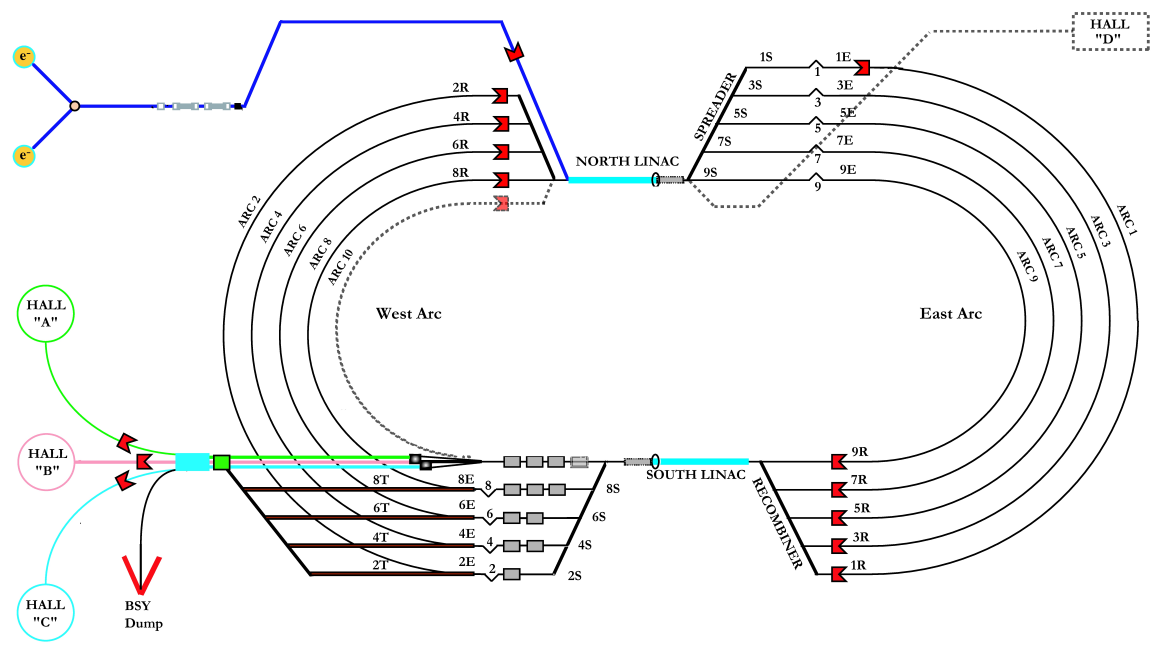

Figure 1.1: Diagram of CEBAF Layout

The beam at this facility starts in the Injector, which is located in the upper- 
left of Figure 1.1. It is here that three $499 \mathrm{MHz}$ lasers create a beam of electrons by striking a cathode. This cathode is held at a potential of $130 \mathrm{kV}$, and consists of a wafer of Gallium Arsenide. Next, the electrons are accelerated through some focusing solenoids and spin rotators, a pre-buncher, a $\sim 400 \mathrm{keV}$ roomtemperature copper cavity, and then two 5 -cell superconducting radio frequency (SRF) cavities. Finally, they are accelerated to up to $67 \mathrm{MeV}$ using two 8cavity SRF cryomodules. At this energy, the electrons are optically matched and injected into the North Linac (NL).

In the NL, the electrons are accelerated to $667 \mathrm{MeV}$ through twenty cryomodules containing eight 5-cell SRF cavities. At the end of the North Linac, the beam reaches a region called the Spreader. In the Spreader, the different energy beams are vertically separated by large dipole magnets; the lowest-energy, firstpass beam is steered toward the topmost arc, Arc 1, while the highest-energy beam is steered toward the lowest arc, Arc 9. Part of the West Arc, which is nearly identical to the East Arc, can be seen in Figure 1.2. Each pass then follows its respective arc around a $180^{\circ}$ bend to the Recombiner region. The Recombiner, the beginning of which can be seen in Figure 1.3, is a mirror-image of the Spreader, and recombines the separate passes back into a single beamline. The section consisting of the Spreader, Arc, and Recombiner does not increase beam energy, as there are no accelerating cavities. It is also isochronous and achromatic, meaning that all electrons will take the same amount of time to travel the same distance independent of energy, and that the position and angle of the beam at the exit of this region is independent of energy.

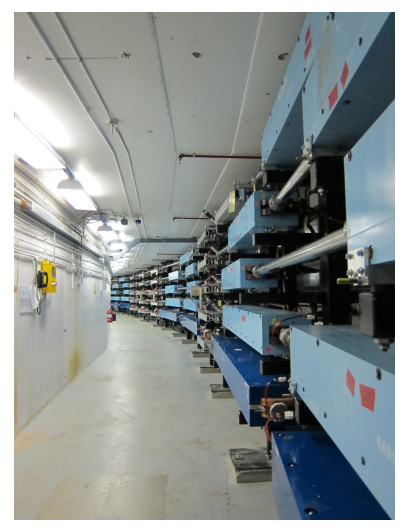

Figure 1.2: West Arc

After exiting the Recombiner, the beam is then accelerated in the South Linac (SL) to $1267 \mathrm{MeV}$ in the same manner as the NL. The beam then enters the West Spreader region, and can either continue around the West Arc, or be extracted to any or all of the experimental halls. The extraction occurs between the spreader and arc and is accomplished with the use of RF separator cavities, which operate at $499 \mathrm{MHz}$, and septum magnets. These RF cavities are used to deflect the correct energy beam to the experimental hall in which 


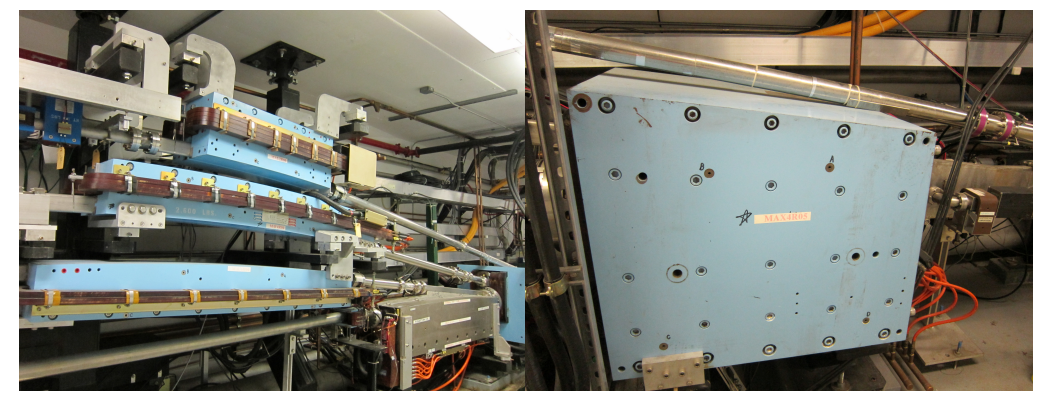

Figure 1.3: Beginning of the Recombiner and a Recombiner Dipole

it is required. Electrons which are to be accelerated to higher energies are then sent into the arc, and recirculated through the machine in the manner which has been described in order to gain momentum. To be sure that the higher-pass beams arrive at the crest of the accelerating RF wave, a series of three dipole magnets, arranged in a three-element symmetric chicane, called doglegs are used to steer the beam and vary the path by up to $1 \mathrm{~cm}$, which corresponds to $18^{\circ}$ for the $1497 \mathrm{MHz}$ wave used at CEBAF.

\subsection{Magnetic Control of Beam Optics}

The optics of the electron beam at Jefferson Lab are controlled using approximately 2800 magnets of varying types. In general terms, quadrupole magnets are used as lenses to focus or defocus the beam, while dipole magnets are used to control the trajectory of the beam by providing steering control. This section will discuss some of the basic principles of some of the magnets used in CEBAF. [19]

\subsubsection{Defining Magnetic Fields}

In order for an accelerator to successfully transport particles to an experimental hall, precise control of the envelope and trajectory of the particle beam must be maintained. At CEBAF, the particle beam is made up entirely of electrons which, due to Coulomb forces, are repelled from each other. More importantly, it is impossible to create a unidirectional beam from an infinitely small spot at the gun. The beam divergence must be controlled to keep the electrons travelling in the same direction. In order to accomplish this, quadrupole magnets are precisely placed throughout the machine in a design lattice. This design lattice is based upon a pattern called a FODO lattice, which refers to the focusing of the quadrupoles in a plane. The " $F$ " refers to a focusing quadrupole, the "D" refers to a defocusing quadrupole, and the "O" refers to a drift. The electromagnetic forces of the quadrupoles act as a restoring force for the particles, and originate from the classical Lorentz equation. A quadrupole that focuses in the $x$ plane 
will defocus in the $y$ plane, so this pattern allows for control of the beam in both planes.

Starting with the Lorentz equation:

$$
\vec{F}=e[\vec{E}+\vec{v} \times \vec{B}]=\frac{d(\gamma m \vec{v})}{d t},
$$

one can see that at relativistic momenta $(\vec{v} \rightarrow c)$, the electric field contribution (from negligible Coulomb repulsion) will be much smaller than that of the magnetic field. Setting the electric field component to zero, the magnetic components become:

$$
\begin{aligned}
& \frac{d\left(\gamma m v_{x}\right)}{d t}=e\left[v_{y} B_{z}-v_{z} B_{y}\right] \\
& \frac{d\left(\gamma m v_{y}\right)}{d t}=e\left[v_{z} B_{x}-v_{x} B_{z}\right] \\
& \frac{d\left(\gamma m v_{z}\right)}{d t}=e\left[v_{x} B_{y}-v_{y} B_{x}\right] .
\end{aligned}
$$

One can set the $B_{z}$ term to zero because the length of the magnets are much larger than their apertures. This approximation is known as a hard-edge approximation, and is valid because the transverse fields ( $x$ and $y$ planes) are much greater than the longitudinal field ( $z$ plane). In setting $B_{z}=0$, and describing the time component in terms of longitudinal velocity and magnet length, $\Delta t=\frac{L}{v_{z}}$, the change in transverse momentum can be written as

$$
\begin{aligned}
\Delta\left(\gamma m v_{x}\right) & =|e|\left[v_{z} B_{y}\right] \frac{L}{v_{z}} \\
\Delta v_{x} & =\frac{|e|}{\gamma m} B_{y} L .
\end{aligned}
$$

The relationship between the transverse and longitudinal velocity goes as, $v_{x}=$ $v_{z} \tan \theta$, where $v_{z} \gg v_{x}$. Using the small angle approximation, $\Delta \theta=\frac{\Delta v_{x}}{v_{z}}$, one can see that the change in the angle is inversely proportional to the longitudinal momentum of the beam:

$$
\Delta \theta=\frac{|e|}{\gamma m v_{z}} B_{y} L
$$

In terms of units, Equation 1.7 can be written as

$$
\Delta \theta[\text { radians }]=2.9979 \times 10^{-4} \frac{B_{y}[\text { gauss }] L[\mathrm{~cm}]}{p_{z}[\mathrm{MeV} / \mathrm{c}]} .
$$

If we define the momentum rigidity as $B \rho=\frac{p_{z}}{e}$, and the radius of curvature, $R$, as $\frac{\Delta \theta}{L}=\frac{1}{R}$, we can then set $\frac{1}{R}=\frac{1}{B \rho} B_{y}$. The transverse size of the beam is very small compared to the radius of curvature. This means that we can use a power series to expand the magnetic field, giving:

$$
\frac{1}{B \rho} B_{y}(x)=\frac{1}{B \rho}\left[B_{0}+\frac{d B_{y}}{d x} x+\frac{1}{2 !} \frac{d^{2} B_{y}}{d x^{2}} x^{2}+\frac{1}{3 !} \frac{d^{3} B_{y}}{d x^{3}} x^{3}+\cdots\right] .
$$


One can then define the coefficients in Equation 1.8 as constants:

$$
\begin{aligned}
\frac{1}{R} & =\frac{B_{0}}{B \rho}, \\
k & =\frac{1}{B \rho} \frac{d B_{y}}{d x}, \\
m & =\frac{1}{B \rho} \frac{d^{2} B_{y}}{d x^{2}}, \\
o & =\frac{1}{B \rho} \frac{d^{3} B_{y}}{d x^{3}} .
\end{aligned}
$$

The first term, from Equation 1.9, is the dipole component of the field. This field is responsible for beam steering. The second term, from Equation 1.10, is the quadrupole component of the field. This field is responsible for the focusing and defocusing of the particle beam. The next two terms, $m$ and $o$, are the sextupole and octupole field terms, respectively. These are nonlinear terms used for chromatic compensation and field error compensation, respectively. The work of this dissertation focuses on the linear optics of the machine, thus the dipole and quadrupole fields are of primary concern.

\subsubsection{Dipole Magnets}

Dipole magnets are used primarily as bending and steering control in accelerators. They vary in size from small corrector dipoles to the large dipoles used in the bending arcs and Spreaders and Recombiners. Referring back to Figure 1.3 , one can see some examples of dipoles. On the left are horizontally bending dipoles, and on the right is one of the large vertically bending dipoles used in the Recombiner. In Figure 1.4, the small blue magnet on the left is a vertical corrector dipole.

From Ampere's Law, and the fact that the current density is zero in the beam pipe, one can determine the shape of the transverse field within a magnet by first defining the magnetic flux density in a plane as

$$
B_{y}(x, y)=G_{y}(x)+f(y),
$$

where $G_{y}(x)$ is the field in the $y$ direction as one moves in the $x$ direction, and $f(y)$ is the field dependence on the $y$-coordinate. Thus, the potential is

$$
\begin{aligned}
\Phi(x, y) & =\int B_{y} d y \\
& =G_{y}(x) y+\int f(y) d y .
\end{aligned}
$$




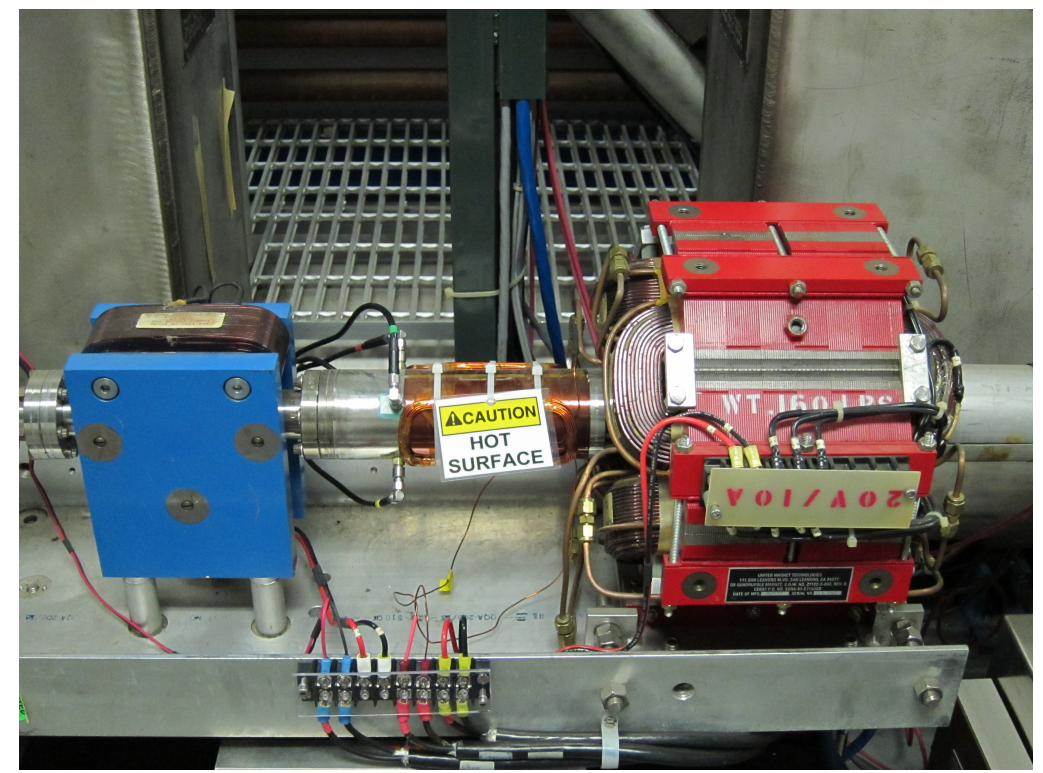

Figure 1.4: Corrector Dipole, BPM, Quadrupole in between Cryomodules

Using Laplace's equation in two dimensions,

$$
\begin{aligned}
\nabla^{2} \Phi & =\frac{\partial^{2} \Phi}{\partial x^{2}}+\frac{\partial^{2} \Phi}{\partial y^{2}} \\
& =\frac{d^{2} G_{y}(x)}{d x^{2}} y+\frac{d f(y)}{d y} \\
& =0 .
\end{aligned}
$$

Therefore, solving for $f(y)$ and substituting back into Equation 1.14 (and integrating), the potential is then

$$
\Phi(x, y)=G_{y}(x) y-\frac{1}{6} \frac{d^{2} G_{y}(x)}{d x^{2}} y^{3} .
$$

Looking at the expansion in Equation 1.8, one can find the correct values of $G_{y}(x)$ and $\frac{d^{2} G_{y}(x)}{d x^{2}}$ for the different types of magnets. Once these values are found, the gradient of the potential is taken, yielding the field distribution in the magnetic aperture.

For a dipole, one investigates the relationship in Equation 1.9, which is constant in the $y$ direction. Thus, for a dipole,

$$
\Phi(x, y)=B_{0} y,
$$




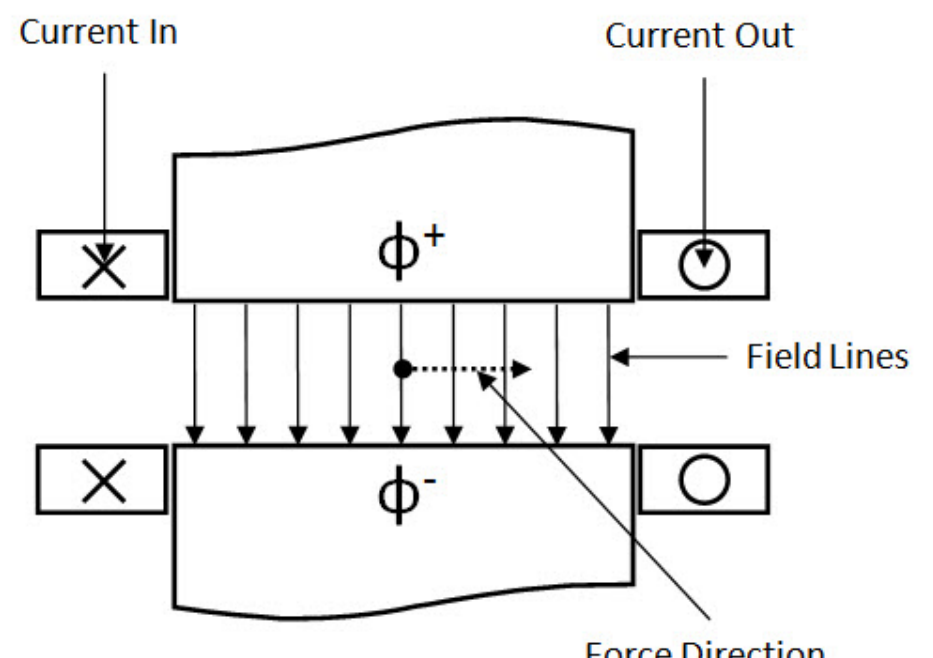

Figure 1.5: Schematic of Dipole [19]

which yields the fields:

$$
\begin{aligned}
& B_{x}(x, y)=\frac{\partial\left(B_{0} y\right)}{\partial x}=0, \\
& B_{y}(x, y)=\frac{\partial\left(B_{0} y\right)}{\partial y}=B_{0} .
\end{aligned}
$$

Looking at Equation 1.19 and Figure 1.5, it becomes apparent how the dipole field acts to steer or bend the beam.

To show the field dependence on the current in the coils and the aperture, one starts with Ampere's Law, $\oint \vec{H} \cdot d \vec{s}=I_{\text {total }}$. Here, $I_{\text {total }}=n I$, where $n$ is the number of conductors, as shown in Figure 1.6. Therefore, Ampere's Law can be rewritten as

$$
\oint \vec{H} \cdot d \vec{s}=n I=H_{F e} l_{F e}+H_{0} h .
$$

One can relate the permeability of free space to the permeability of iron as $\mu_{r}=\frac{\mu_{F e}}{\mu_{0}} \gg 1$. Thus,

$$
\begin{aligned}
\oint \vec{H} \cdot d \vec{s} & =n I=H_{F e} l_{F e}+H_{0} h \\
& \approx H_{0} h \\
H_{0} & =\frac{n I}{h} .
\end{aligned}
$$

Since $B_{0}=\mu_{0} H_{0}$, the field of a dipole can be written as

$$
B_{0}=\frac{\mu_{0} n I}{h} \text {. }
$$




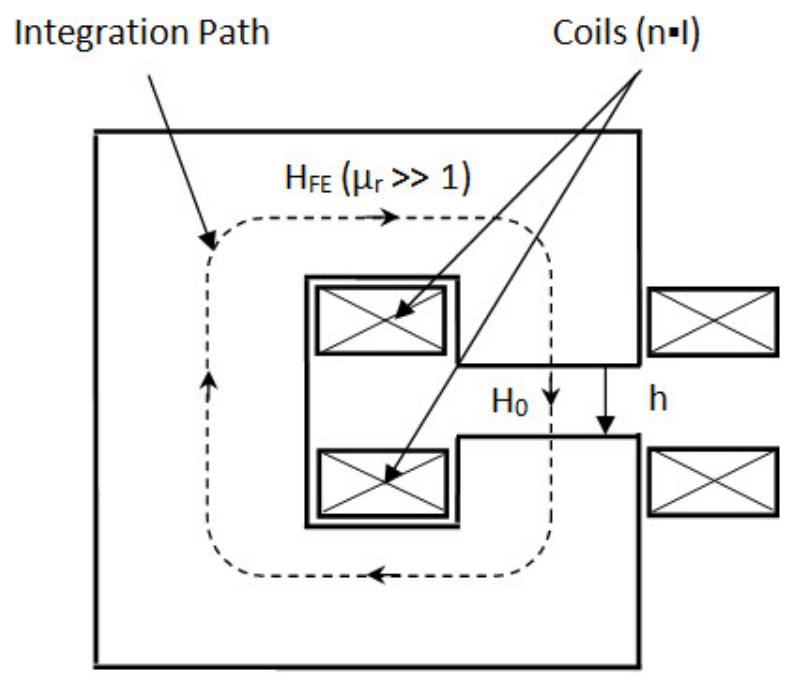

Figure 1.6: C-type Dipole with Integration Path [19]

Thus, the dipole field is inversely proportional to the gap between the poles, and directly proportional to the current through the coils of wire.

\subsubsection{Quadrupole Magnets}

Looking back again at Equation 1.10 and Figures 1.4 (the red magnet) and 1.7, one sees that the strength of the field decreases linearly as one moves along the $x$-axis closer to the center of the quadrupole. Defining $g=\frac{d B_{y}}{d x}$, and substituting into Equation 1.16, the potential of the quadrupole is then

$$
\Phi(x, y)=g x y .
$$

Taking the derivatives for each plane, the quadrupole fields are:

$$
\begin{aligned}
& B_{x}(x, y)=g y \\
& B_{y}(x, y)=g x .
\end{aligned}
$$

The force on an electron moving with velocity $\vec{v}=v_{0} \hat{z}$ is given by

$$
\begin{aligned}
\vec{F} & =q \vec{v} \times \vec{B}=-e v_{0} g(\vec{y}-\vec{x}), \\
\vec{F} & =e v_{0} g(\vec{x}-\vec{y}) .
\end{aligned}
$$

Thus, for positive (negative) $g$, the electron experiences a defocusing (focusing) force in the $\hat{x}(\hat{y})$ direction.

The dependence on the quadrupole aperture and coil current is found in a similar manner as for a dipole. The difference is in the path of integration, 


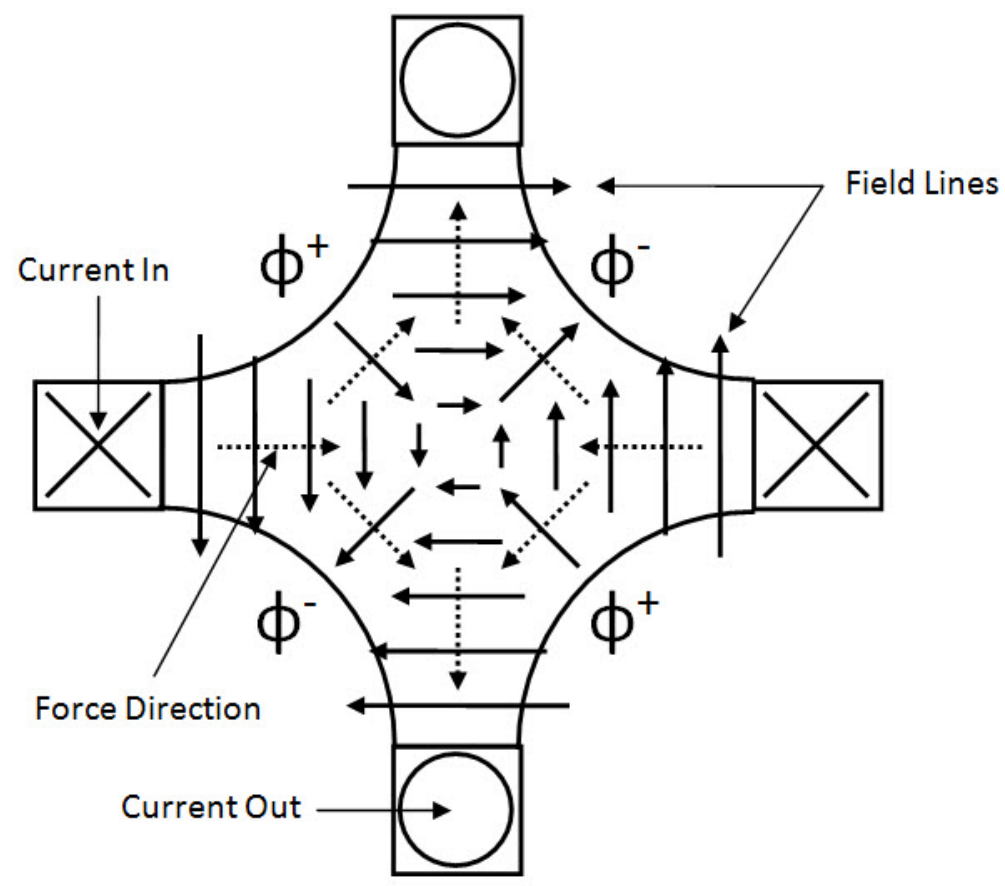

Figure 1.7: Schematic of Quadrupole [19]
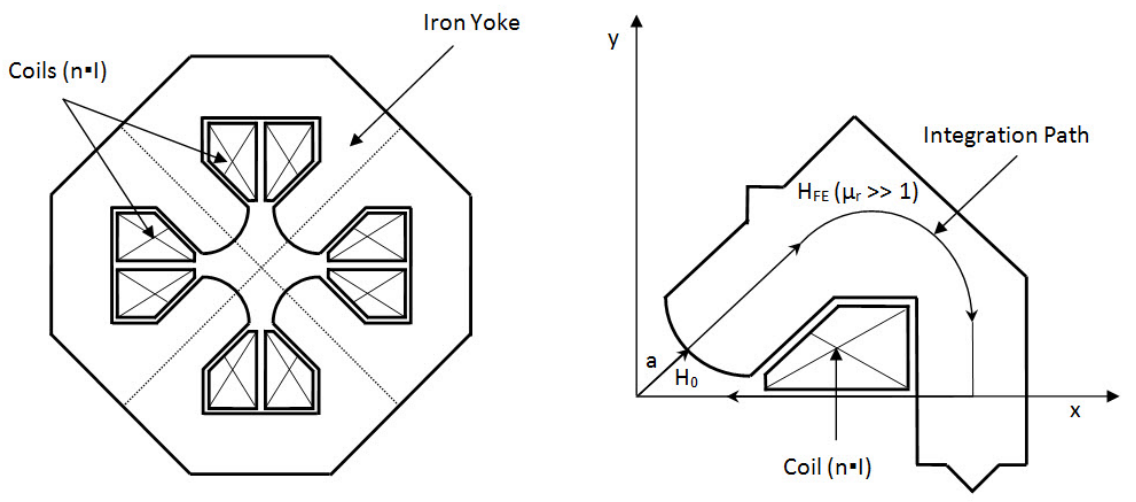

Figure 1.8: Integration Path of Quadrupole [19] 
shown in Figure 1.8. There are three different segments of the integration path for a quadrupole. The first is path within the steel, which is small compared to that of the gap. The second is along the $x$-plane, where $B \cdot d s=0$ because it is perpendicular to the field. Finally, there is a part which connects the center of the quadrupole to the pole face. In this third path, $H_{0}=\frac{g}{\mu_{0}} r$, where $r=\sqrt{x^{2}+y^{2}}$. Integrating from the center of the aperture to the pole tip,

$$
\begin{aligned}
\int_{0}^{a} H_{0} d r & =\int_{0}^{a} \frac{g}{\mu_{0}} r d r \\
& =\frac{g a^{2}}{2 \mu_{0}}=n I .
\end{aligned}
$$

Solving for $g$, and setting $g=\frac{\partial B}{\partial x}$, the quadrupole field can then be expressed as

$$
B_{y}=\frac{2 \mu_{0} n I x}{a^{2}} .
$$

Thus, the field depends linearly upon the $x$-coordinate and the current, and is inversely proportional to the square of the distance from the center of the aperture to the pole tip.

\subsection{Beam Position Monitors [9, 19]}

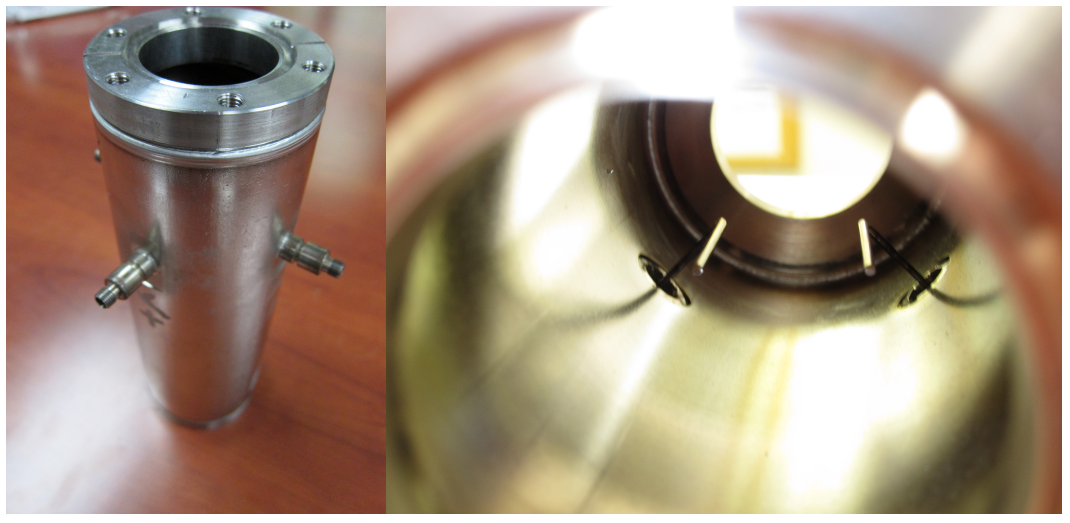

Figure 1.9: Beam Position Monitor (BPM)

Beam position monitors (BPMs) are used to measure the trajectories of the beam throughout the beamline. They are placed in carefully-selected areas of the beamline, and measure the position of the beam with respect to the center of the beam pipe. This section will discuss some basic concepts of how the BPMs work. 
The concept behind a BPM is that the electric field produced by the beam will induce a charge on an insulated metal plate, which can then be measured. This information can then be used to find the position of the beam in the beam pipe. Particle beams are made up of small collections of electrons, referred to as bunches, which are time-dependent. This means that the electric field of the beam is also time-dependent, and that an alternating current (AC) signal will be seen on the plates in the BPM. In order to determine the position of the beam centroid, four pickup plates are installed $90^{\circ}$ apart around the beam pipe. The difference in signals measured on plates opposite each other yields the transverse position of the center of charge of the beam.

In general, the electric fields produced by the charged particle beam couple to the pickups. For relativistic particles, the transverse field components as seen in the lab frame increase when compared to the rest frame of the beam. This can be described by the Lorentz transformation from the beam's rest frame at time $t^{\prime}$ to the lab's frame at time $t$ for the transverse field component,

$$
E_{\perp l a b}(t)=\gamma E_{\perp \text { beam }}\left(t^{\prime}\right),
$$

where the Lorentz factor is defined as $\gamma=\frac{1}{\sqrt{1-\left(\frac{v}{c}\right)^{2}}}$. The transverse field component increases with the Lorentz factor, and even at moderate $\left(\frac{v}{c} \equiv \beta>0.5\right)$ beam velocities can be approximated by the transverse electric and magnetic (TEM) field distribution. The electric and magnetic field vectors are perpendicular to the direction of particle propagation in a TEM mode, and a TEM wave can be substituted for the beam for most calculations. It is assumed that the power carried by the beam is much greater than the power coupled out by the detector.

There are several basic types of BPMs which are used for different types of accelerators and beam parameters. Jefferson Lab uses what is known as a stripline style BPM, one of which is pictured in Figure 1.9. For a more indepth discussion of the different styles of BPMs, as well as why one would use a specific style, see the work of Peter Forck, et al. [9] Jefferson Lab uses several configurations of stripline BPMs. This dissertation will focus mainly on what is called an M20, which has antenna-style pickups and is located throughout ARC1 and ARC2, as well as the Extraction Region. This will be the style of BPM described in this section. Differences with other BPMs will be discussed as needed throughout other sections and chapters in this work.

As described previously, the particle beam will couple with the pickups of the BPM. At CEBAF, the electric field created by the electron beam will couple with the $\frac{1}{4}$-wave antenna pickups in the BPM. To find the transverse beam position, one takes the difference of the voltages induced on antennas located $180^{\circ}$ apart, divides by the sum of these voltages, and multiplies by a proportionality term:

$$
r \propto \frac{V^{+}-V^{-}}{V^{+}+V^{-}} .
$$

For a perfectly centered beam, the difference between the two induced voltages would be exactly zero, and the position, $r$, would also equal zero. The proportionality constant, $k$, depends upon the geometric design of the BPM. For the 

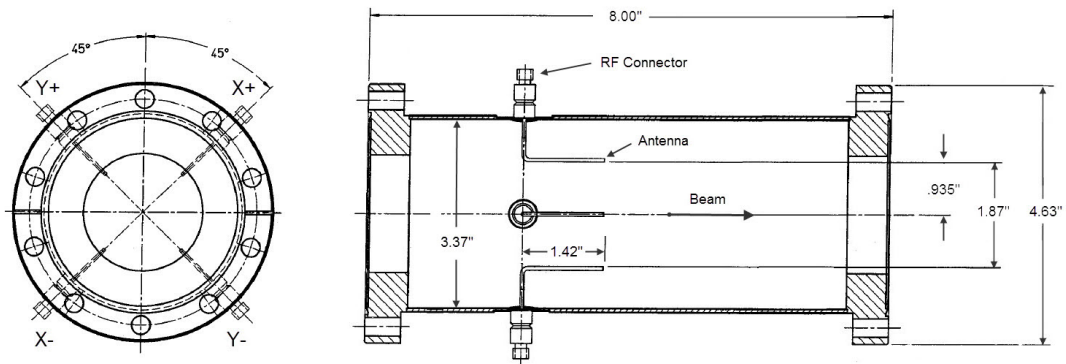

Figure 1.10: Schematic of BPM used at CEBAF [19]

M20 BPM pictured in the schematic in Figure 1.10, $k=25.56 \mathrm{~mm}$. Additionally, due to possible electronic and mechanical mismatches between the different channels, another constant, $\alpha$, must be introduced. The position can now be written

$$
r=k \frac{V^{+}-\alpha V^{-}}{V^{+}+\alpha V^{-}} .
$$

As seen in Figure 1.10, the four pickups of the BPM are divided into $X$ and $Y$ pairs, each pair having antennas corresponding to positive and negative position offsets. The four antenna wires are labeled $X^{+}, X^{-}, Y^{+}$, and $Y^{-}$. The proportionality constant, $\alpha$, is found for each plane through a calibration procedure. To find this constant in the $Y$-plane, the beam is turned off and an RF calibration signal is put through the $X^{-}$wire, and readings are taken on the $Y$ wires with this calibration signal on $\left(U^{ \pm}\right)$, and then again with the signal off $\left(U_{o f f}^{ \pm}\right)$. The opposite is done to find the constant for the $X$-plane wires. These constants are related to the signals as

$$
\begin{aligned}
& \alpha_{x}=\frac{X^{+}-X_{o f f}^{+}}{X^{-}+X_{o f f}^{-}}, \\
& \alpha_{y}=\frac{Y^{+}-Y_{o f f}^{+}}{Y^{-}+Y_{o f f}^{-}} .
\end{aligned}
$$

The position of the beam centroid in the beampipe for a single plane can now be described in general coordinates as

$$
U=k \frac{\left(U^{+}-U_{o f f}^{+}\right)-\alpha_{u}\left(U^{-}-U_{o f f}^{-}\right)}{\left(U^{+}-U_{o f f}^{+}\right)+\alpha_{u}\left(U^{-}-U_{o f f}^{-}\right)} .
$$

This position measurement represents the position of the beam in each plane for the BPM frame. However, due to the damage that could occur to the antennae if they were struck with the synchrotron light that is emitted by the electron beam in either bend plane, or the large photoelectric interference that could occur on the outboard side of the bend, the BPM frame is actually rotated $45^{\circ}$ 
at Jefferson Lab. Therefore, in order to find the position of the beam centroid in the lab frame, the BPM frame must be rotated back $45^{\circ}$. Thus, if $U=X_{\text {rot }}$ and $U=Y_{\text {rot }}$ for each plane, the rotation from BPM frame to lab frame is achieved by a simple coordinate rotation:

$$
\begin{aligned}
X & =\frac{1}{\sqrt{2}}\left(X_{\text {rot }}-Y_{\text {rot }}\right) \\
Y & =\frac{1}{\sqrt{2}}\left(X_{\text {rot }}+Y_{\text {rot }}\right) .
\end{aligned}
$$

For further information about the beam position monitors at Jefferson Lab, including the manner in which they are calibrated, nonlinearities in measurements at large radii, and some of the related electronics, the reader is directed to the dissertation of Michael Spata [19]. 


\section{Chapter 2}

\section{Accelerator Physics Background}

\subsection{Beam Dynamics}

This section will discuss some of the fundamental concepts of beam dynamics. First, a brief description of phase space will be discussed, followed by a discussion of the matrix formalism. The chapter will finish with a description of the Courant-Snyder, or Twiss, parameters. For more in-depth discussion on any of these topics, see any of the standard textbooks on Accelerator Physics $[6,15,25,26,27]$.

\subsubsection{Phase Space}

Studying the statistical properties of a collection of particles is both more practical and more useful than studying the properties of a single particle. In order to do this, one investigates the dynamics of the particles in six dimensional phase space, which is represented by the coordinates and momenta, $\left(x, p_{x}, y, p_{y}, \sigma, E\right)$. Here $\mathrm{x}$ and $\mathrm{y}$ are positions, $\frac{p_{x}}{p_{0}} \approx x^{\prime}$ and $\frac{p_{y}}{p_{0}} \approx y^{\prime}$ show the transverse momenta, $\frac{c p_{0}}{\beta}=E_{0}$ is the ideal particle energy, $\sigma$ is the coordinate along the trajectory, and $E$ is the particle energy. Alternatively, $E$ is often represented as an energy deviation, or a relative energy deviation with respect to a reference, or ideal particle. Additionally, if beam energy is constant, it is common to use the slope of the trajectories, $x^{\prime}$ and $y^{\prime}$, rather than the transverse momenta, as they are proportional to the momenta and also small, thus allowing one to let $\sin x^{\prime} \approx x^{\prime}$.

\subsubsection{Matrix Formalism [15, 25, 26, 27]}

The theory of strong focusing, as developed by Courant, Snyder, Livingston, and Christofilos, [7] is based on the use of quadrupole magnets for focusing. Use of this approach allows for a smaller magnetic aperture, as the strong focusing of 
the quadrupoles restricts the particles to a much smaller cross-sectional area. The following discussion will show the common treatment of the physics for strong focusing machines, such as CEBAF. The focus of the discussion will be on linear optics. Additionally, the horizontal and vertical planes will, for the most part, be treated independently, as there exists only small cross coupling at CEBAF.

For a beam bent in the horizontal, $x$, plane, one can express the bending and quadrupole magnetic fields as

$$
\begin{aligned}
& B_{x}=-g y, \\
& B_{y}=B_{y 0}+g x,
\end{aligned}
$$

where $B_{y 0}$ is the dipole field, and $g$ is the gradient of the quadrupole field. Defining $\kappa$ as the local curvature vector with $\kappa_{i}=\frac{1}{\rho_{i}}$ ( $\rho$ is the local bending radius), and $k=\frac{e g}{p}=\frac{1}{l f}$ as the energy independent focusing strength ( $l$ is the particle's path length in the magnetic field and $f$ is the magnet's focal length), one can obtain the linear approximation of the equations of motion (Hill's Equation):

$$
\begin{aligned}
x^{\prime \prime}+\left(k_{0}+\kappa_{0 x}^{2}\right) x & =0, \\
y^{\prime \prime}-k_{0} y & =0 .
\end{aligned}
$$

The magnetic strength parameters depend upon the location of the magnets along the beamline, thus depending upon the $z$ coordinate. These equations are useful in finding the focusing power along the beamline. By integrating them over a small distance along the beamline, the deflection angle is directly found:

$$
\int y^{\prime \prime} d z=y^{\prime}-y_{0}^{\prime}=\delta
$$

Defining $\delta$ to be the deflection angle;

$$
\delta=-\frac{r}{l}=-\frac{l}{\rho},
$$

and taking $\int k_{0} y d z \approx k_{0} y \Delta z$, general expressions for the focal length of the magnetic gradient fields can written:

$$
\begin{aligned}
\frac{1}{f_{x}} & =k_{0} \Delta z=\frac{e}{c p} \frac{\partial B_{y}}{\partial x} \Delta z \\
\frac{1}{f_{y}} & =-k_{0} \Delta z=-\frac{e}{c p} \frac{\partial B_{x}}{\partial y} \Delta z
\end{aligned}
$$

Knowledge of any segment's field gradient can yield either the segment's focusing power or its equations of motion. All of the focusing in a segment, both from the bending magnets and the quadrupoles, can be combined:

$$
K(z)=k_{0}(z)+\kappa_{0 x}^{2}(z) .
$$


A very informative and convenient way to describe the trajectories of particles through a beamline is through use of the matrix formulation. The matrix formulation I will describe uses the hard edge model, which assumes that the magnet strength parameters within a magnet are constant, and fringe fields and other such factors are ignored. In this model, the magnet parameter, $K$, varies in a stepwise fashion, as the magnetic field is either present at a given location, or not present. Additionally, $K$ is a constant, and the equations of motion can be represented as a harmonic oscillator in matrix form:

$$
\left[\begin{array}{c}
u(z) \\
u^{\prime}(z)
\end{array}\right]=\left[\begin{array}{ll}
C_{u}(z) & S_{u}(z) \\
C_{u}^{\prime}(z) & S_{u}^{\prime}(z)
\end{array}\right]\left[\begin{array}{c}
u_{0} \\
u_{0}^{\prime}
\end{array}\right]
$$

where $u$ is a generalized coordinate for either $x$ or $y$. $C$ and $S$ are cosine and sine functions and are defined as:

$$
\begin{gathered}
K>0: C(z) \equiv \cos (\sqrt{K} z) \text { and } S(z)=\frac{1}{\sqrt{K}} \sin (\sqrt{K} z) \\
K<0: C(z) \equiv \cosh (\sqrt{|K|} z) \text { and } S(z)=\frac{1}{\sqrt{|K|}} \sinh (\sqrt{|K|} z) .
\end{gathered}
$$

The $2 \times 2$ generalized matrix can also be expanded in such a way that both $x$ and $y$ terms are shown:

$$
\left[\begin{array}{c}
x(z) \\
x^{\prime}(z) \\
y(z) \\
y^{\prime}(z)
\end{array}\right]=\left[\begin{array}{cccc}
C_{x}(z) & S_{x}(z) & 0 & 0 \\
C_{x}^{\prime}(z) & S_{x}^{\prime}(z) & 0 & 0 \\
0 & 0 & C_{y}(z) & S_{y}(z) \\
0 & 0 & C_{y}^{\prime}(z) & S_{y}^{\prime}(z)
\end{array}\right]\left[\begin{array}{c}
x_{0} \\
x_{0}^{\prime} \\
y_{0} \\
y_{0}^{\prime}
\end{array}\right] .
$$

The matrix terms that are null in Equation 2.7 represent cross-coupling terms. Additionally, this matrix can be further expanded to include terms for energy and dispersion.

In a drift, where there exists no focusing, as well as in areas of weak bending, where $\frac{1}{\rho_{0}^{2}} \approx 0$ and $k_{0}=0$, the focusing parameter, $K$, is zero, and the matrix is then:

$$
\left[\begin{array}{c}
u(z) \\
u^{\prime}(z)
\end{array}\right] \approx\left[\begin{array}{cc}
1 & z-z_{0} \\
0 & 1
\end{array}\right]\left[\begin{array}{l}
u_{0}\left(z_{0}\right) \\
u_{0}^{\prime}\left(z_{0}\right)
\end{array}\right]
$$

If one lets $l=z-z_{0}$, then the drift transformation matrix can be represented by

$$
M_{d}=\left[\begin{array}{ll}
1 & l \\
0 & 1
\end{array}\right] .
$$

For a pure quadrupole, the bending term is $\frac{1}{\rho_{0}}=0$, but the field gradient will be either positive or negative, depending on whether the quadrupole is focusing or defocusing in the reference frame. For a focusing quadrupole, $k_{0}>0$ and in this limit, Equation 2.6 becomes 


$$
\left[\begin{array}{c}
u(z) \\
u^{\prime}(z)
\end{array}\right]=\left[\begin{array}{cc}
\cos \Psi & \frac{1}{\sqrt{k_{0}}} \sin \Psi \\
-\sqrt{k_{0}} \sin \Psi & \cos \Psi
\end{array}\right]\left[\begin{array}{c}
u\left(z_{0}\right) \\
u^{\prime}\left(z_{0}\right)
\end{array}\right]
$$

where $\Psi=\sqrt{k_{0}}\left(z-z_{0}\right)$. This matrix holds so long as $l$ is within the confines of the quadrupole length. This means that, for a focusing quadrupole of length $l$ and strength $k_{0}$, the transfer matrix can be described by:

$$
M_{Q F}=\left[\begin{array}{cc}
\cos \varphi & \frac{1}{\sqrt{k_{0}}} \sin \varphi \\
-\sqrt{k_{0}} \sin \varphi & \cos \varphi
\end{array}\right]
$$

where $\varphi=\sqrt{k_{0}} l$. The same is true for a defocusing quadrupole, but the signs are switched such that the transfer matrix can be described by:

$$
M_{Q D}=\left[\begin{array}{cc}
\cosh \varphi & \frac{1}{\sqrt{k_{0}}} \sinh \varphi \\
-\sqrt{k_{0}} \sinh \varphi & \cosh \varphi
\end{array}\right]
$$

To find the transformation matrix for a section of beam line which contains both quadrupoles and driftspace, one simply multiplies the transfer matrices in reverse order of occurrence.

\subsubsection{The Twiss Parameters [27]}

Attributes of the beam can be described by the Twiss parameters. These terms describe the area in phase space that is occupied by the particle beam. $\alpha=\frac{-\beta^{\prime}}{2}$ is related to the distance to the geometric focus from a lens. When it is positive, the beam is convergent, and when it is negative, the beam is divergent. $\beta$ is an amplitude function, and $\sqrt{\beta \epsilon}$ is the radius of the beam, where $\epsilon$ is the emittance. The emittance is proportional to the area that a set of beams will occupy in phase space, as Area $=\pi \epsilon$. $\gamma$ relates the focusing distance and the amplitude, and is defined as $\gamma \equiv \frac{1+\alpha^{2}}{\beta}$. These terms are all interrelated, as demonstrated by the phase ellipse in Figure 2.1.

One can create a transfer matrix for the beam in terms of the Twiss parameters as follows:

$$
M=\left[\begin{array}{cc}
\sqrt{\frac{\beta_{2}}{\beta_{1}}}\left(\cos \Delta \psi+\alpha_{1} \sin \Delta \psi\right) & \sqrt{\beta_{1} \beta_{2}} \sin \Delta \psi \\
-\frac{1+\alpha_{1} \alpha_{2}}{\sqrt{\beta_{1} \beta_{2}}} \sin \Delta \psi+\frac{\alpha_{1}-\alpha_{2}}{\sqrt{\beta_{1} \beta_{2}}} \cos \Delta \psi & \sqrt{\frac{\beta_{1}}{\beta_{2}}}\left(\cos \Delta \psi-\alpha_{2} \sin \Delta \psi\right)
\end{array}\right] .
$$

Equation 2.11 shows how the Twiss parameters effect the evolution of the beam from one point to another. It uses the $\alpha, \beta$, and the phase advance, $\psi$, to transform the coordinates of the beam. The phase advance, which is related to $\phi$ by a transformation into a different coordinate system, is defined as

$$
\Delta \psi=\tan ^{-1}\left(\frac{m_{12}}{\beta_{1} m_{11}-\alpha_{1} m_{12}}\right)
$$

where the $m_{i j}$ terms refer to the specific components in Equation 2.11. 


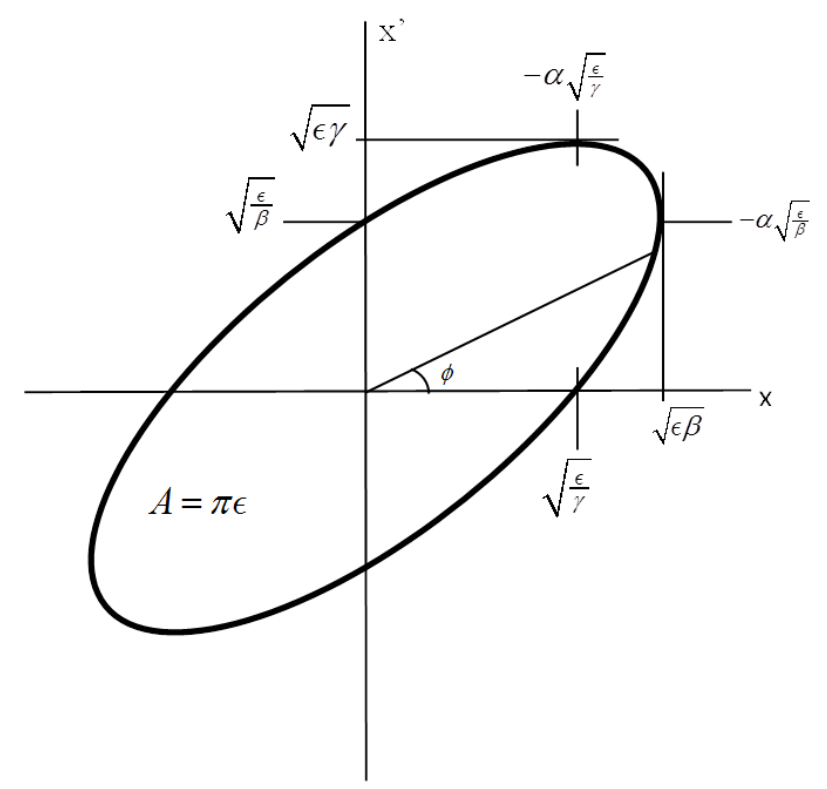

Figure 2.1: Standard Phase Space Ellipse Labeled with Twiss Parameters

It is important to note that the Twiss parameters have different meanings in linear versus circular accelerators. A stable circular machine will have a closed orbit, and any particles injected off of that closed orbit will sequentially trace out a bounded curve in phase space at a given location. This bounded curve defines the Twiss parameters at this location. Therefore, the Twiss parameters in a circular machine are defined by the lattice.

In an open-ended machine, the beam only passes through the system once, and the lattice does not have the same periodic constraints as a circular machine. Instead, the lattice defines the path of the beam and rather than defining the Twiss parameters, it transforms them. In an open-ended machine, the Twiss parameters are an intrinsic property of the beam, as one can see in the RMS definitions below:

$$
\begin{gathered}
\epsilon_{r m s}^{2}=\left\langle x^{2}\right\rangle\left\langle x^{\prime 2}\right\rangle-\left\langle x x^{\prime}\right\rangle^{2}, \\
\beta=\frac{\left\langle x^{2}\right\rangle}{\epsilon_{r m s}}, \\
\alpha=\frac{\left\langle x x^{\prime}\right\rangle}{\epsilon_{r m s}}, \\
\gamma=\frac{\left\langle x^{\prime 2}\right\rangle}{\epsilon_{r m s}} .
\end{gathered}
$$

The Twiss parameters evolve throughout the beamline, and this evolution can be found using a transformation matrix. Using the matrix in Equation 2.11, 
a new $3 \times 3$ matrix can be defined as

$$
\mathcal{M}=\left[\begin{array}{ccc}
m_{11}^{2} & -2 m_{11} m_{12} & m_{12}^{2} \\
-m_{11} m_{21} & m_{11} m_{22}+m_{12} m_{21} & -m_{12} m_{22} \\
m_{21}^{2} & -2 m_{21} m_{22} & m_{22}^{2}
\end{array}\right] .
$$

Here, $\mathcal{M}$ is the transformation matrix for the Twiss parameters themselves:

$$
\left[\begin{array}{l}
\beta \\
\alpha \\
\gamma
\end{array}\right]_{2}=\mathcal{M}\left[\begin{array}{l}
\beta \\
\alpha \\
\gamma
\end{array}\right]_{1}
$$




\section{Chapter 3}

\section{Diagnostic Tools Currently in Use}

At the CEBAF machine, two methods are currently used to characterize and tune the beamline; quadrupole scans, and the Courant-Snyder tool. This chapter will discuss these methods, as well as their strengths and weaknesses.

\subsection{Quadrupole Scan}

One powerful tool for determining the local optical parameters of the CEBAF beamline is the quadrupole scan. Operators will adjust the strength of a quadrupole by some known amount, varying the downstream beam size. Beam profile monitors, also called harps or wire scanners, are used to measure the differential beam size. The relationship between the smallest beam radius to the beam radius at the lens can be used to calculate the uncorrelated angular spread, which I refer to as the emittance angle. Centrally located beams will retain the same emittance angle, regardless of the quadrupole value.

If one were to plot the RMS beam radius as a function of the lens strength, a hyperbola would be seen. Measuring the beam radius at the waist of this hyperbola will result in a sharp peak. This measurement is vulnerable to beam jitter, as the width of the peak is narrow. Measuring away from the beam waist gives a flatter peak, and is more vulnerable to measurement noise.

In order to better explain the way this method works, I will briefly discuss the simplified case where the harp and waist are coincident. The emittance of the beam is related to the beam radius and its angle as $\epsilon=\theta R_{\text {beam }}$. Looking at Figure 3.1, one sees that beams originating at the extremum of the lens will follow the hyperbolic asymptotes, defining the radius of the beam envelope, as well as the near and far field. Beams that originate at the center of the lens will maintain small angles and run near to the hyperbolic curve at the waist. They

define the core of the beam and undergo free expansion. Ballistic expansion of these core beam particles governs the size of the beam. 


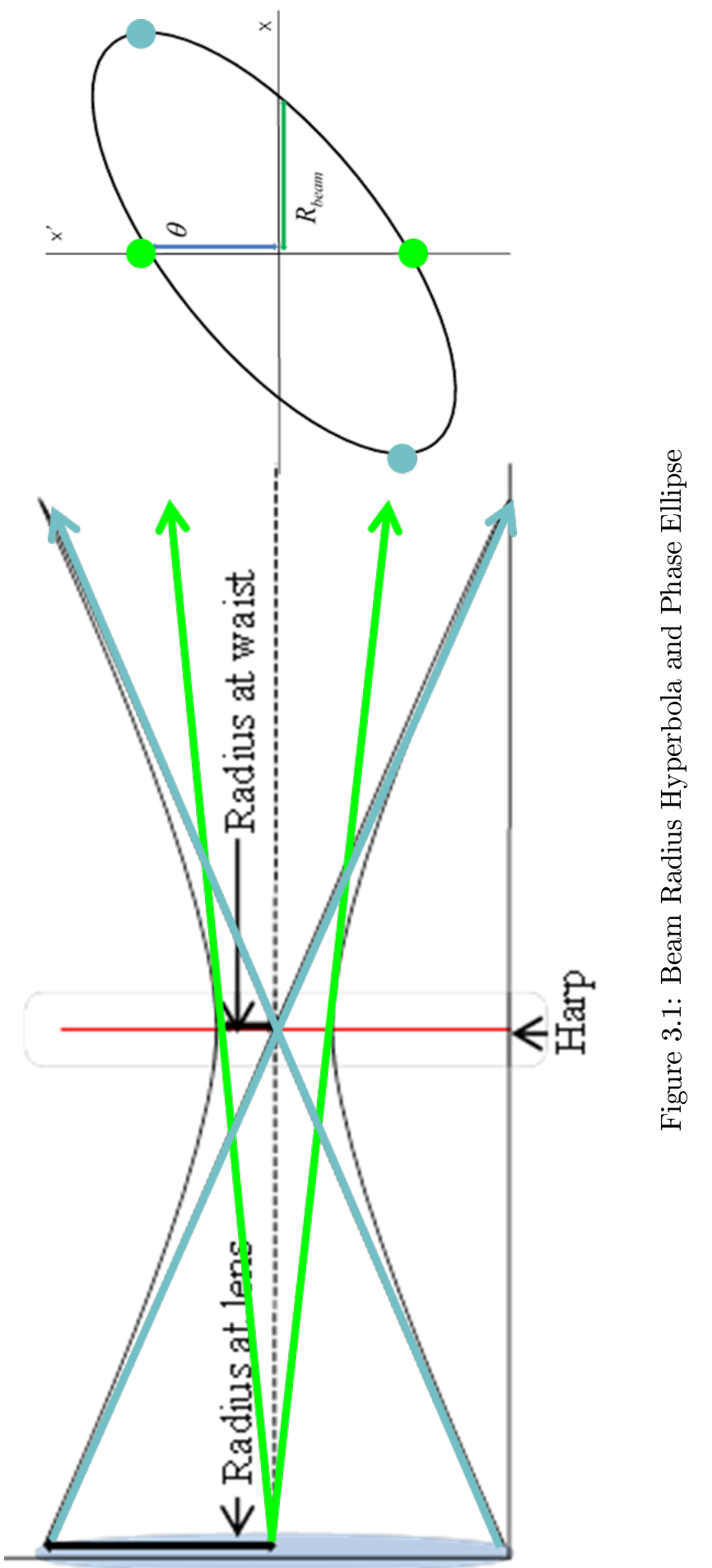


The radius of the beam at the lens is related to the angle and lens strength as $\delta \theta=\frac{R_{\text {lens }}}{f}$. The radius of the beam at the waist has a similar relationship; $\theta \times l=R_{\text {waist }}$, where $l$ is the distance to the waist. Using these relationships, one can see the basic idea of how the emittance is found using this method. The real method, as it is utilized in the machine, uses statistical methods to calculate these parameters using several quadrupole settings.

While this method can be a powerful tool, and is common in many accelerators, there are significant problems with its utilization at CEBAF due to lab-specific limitations. One of the major problems is the sparse coverage of the machine with harps. Another is the high levels of noise the current monitors have due to the size of the wires in relation to the small emittance.

The small emittance of CEBAF is another limitation of this method. Due to the thickness of the wires in our scanners, their accuracy is limited when measuring such a small beam.

The usage protocols of our harps are a further limitation, as a single cycle through the beampipe takes two minutes. Since this scan is invasive, the end users cannot receive beam when these scans are run. Because they are time consuming, the nuclear physics program must be put on hold for a long time during this process. To make matters worse, this measurement only provides information at one part of the beamline. In order to characterize an entire section, several of these invasive, time-consuming measurements must be performed at a series of locations.

\subsection{The Courant-Snyder Method}

One of the methods used to maintain beam envelope matching at CEBAF is a tool called the "Courant-Snyder," named after the authors from the 1950s [7]. This tool (CS) takes position and angle information from two kicks provided at different correctors and compares the values to those in the design optics.

Figure $3.2 \mathrm{a}$ is an example of what the design model says things should look like at a given location in the machine. The red stars indicate the expected trajectory locations for the given corrector kicks from the model at this location. When a beam does not follow the model, the real beam may resemble the blue ellipse in Figure 3.2b, which would represent the real consequence of transport errors before this point. The blue stars on this ellipse indicate where the real trajectories have been measured by a beam position monitor at this location. These measurements are taken at all BPMs in the machine, but this description will focus on one BPM.

Once these points are measured, the CS tool takes the design values for $\alpha$ and $\beta$ and calculates the equivalent Courant-Snyder invariant, $\epsilon=\gamma x^{2}+2 \alpha x x^{\prime}+\beta x^{\prime 2}$ (related to the area of the phase ellipse, which is a constant), at those points. When this occurs, the calculated action associated with this trajectory is not correct, as seen by the green ellipses in Figure 3.2c. This situation represents a mismatch and means that the calculated action varies along the beamline.

When tuning with this tool, adjustments are made to move these values (blue 


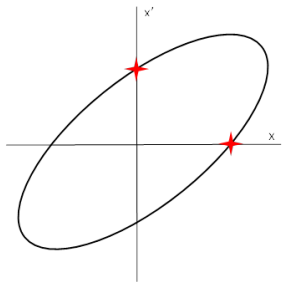

(a) Design

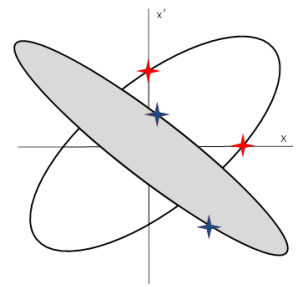

(b) Real Beam

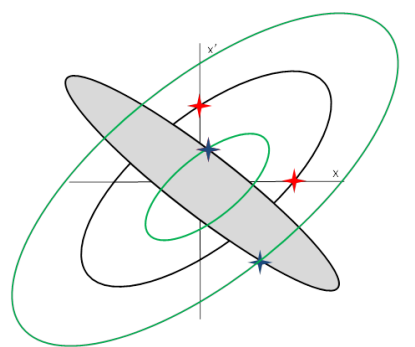

(c) CS Mismatch

Figure 3.2: Courant-Snyder Plots

stars) back onto the ellipse of the design value (red stars). However, the CS tool provides no information about the mismatch; it only detects its presence. This means tuning must be done in an unguided manner.

By design, this procedure does not require any particular cumulative phase advance. While this is a strength much of the time, it can also lead to problems of degeneracy, where the two measured points are indistinguishable from each other. In Figure 3.3, one sees the problems that can occur when there is a severe mismatch of the real beam from the design beam. Because the CS tool does not take into account cumulative phase advance errors, it cannot identify discontinuities, meaning there is no flag for degeneracies, such as represented by the blue stars. When these measured trajectories are so close together, they may be viewed as one point by the CS tool. This problem would easily be identified if knowledge of the phase advance were used.

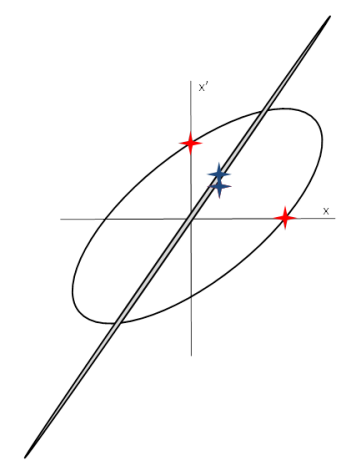

Figure 3.3: Courant-Snyder Degeneracy 


\section{Chapter 4}

\section{A New Tool: rayTrace}

Previously, the current methods used to characterize and tune the CEBAF machine were described, and their strengths and weaknesses were addressed. With the weaknesses these procedures have, a new tool is necessary as the facility prepares to double its current energy, as the errors that are acceptable now will no longer be. A more robust tool is needed.

CEBAF needs a method that is minimally invasive to its nuclear physics program. Users require as much beam time as possible to gather data, so minimizing time lost during characterization and tuning is a key requirement. Additionally, a method is required that can either address the cumulative phase advance errors, or provide a way that they can be ignored without detriment. Furthermore, the new method must be able to characterize the beam locally, but it must also provide global, long-range knowledge of the machine if desired. This means that optical information must be provided in specific places, as well as in a distributed manner.

In this chapter, I present the new, developing method that we refer to as rayTrace. rayTrace is not related to any other program or software that is available publicly.

\subsection{What is rayTrace?}

rayTrace is essentially a real-world simulation, using the CEBAF accelerator to model itself. Considering each plane separately, and assuming minimal crossplane coupling, we use two correctors to steer the beam centroid in a measured, iterative manner, painting the boundary of a design phase ellipse at a downstream launchpoint chosen at a beam position monitor (BPM). Each iterative kick represents one of a family of rays that make up this phase ellipse bound-

ary, and the family of rays together simulates the boundary of a beam in the machine: a "synthetic" beam if you will.

Simultaneously at every downstream BPM from the launchpoint, the position is measured for each kick. This position information, along with knowledge 
of the distance between elements and local, short-range transport optics, allows for the calculation of the Twiss parameters for this beam.

Adjusting two correctors simultaneously allows us to use arbitrary linear combinations of the corrector pair, giving us denser phase space access. We are able to change both the $x$ and $x^{\prime}$ values simultaneously, each new twocorrector kick giving a new point along the perimeter of the phase ellipse. This is repeated until we have the desired number of data points along the ellipse. Once we complete one turn around the ellipse, we repeat this procedure one or more times in order to check for beam drift during the procedure, which would appear as an unclosed ellipse in the data. This provides many data points along the boundary of our "synthetic" beam, and helps to better define the beam's parameters.

\subsection{How rayTrace Works}

\subsubsection{Data Acquisition}

The Accelerator and High Level Applications (AHLA) group at Jefferson Lab provided a useful and flexible graphical user interface (GUI) prototype for the data collection process. This GUI is currently only intended for expert-only use, as it provides many settings and options that a general user would not use. Default values for the settings described below are from the model.

Figure 4.1 is a screenshot of the GUI. When using this interface, the user will first choose the desired plane and then a launchpoint. The GUI then selects two default correctors to use for simultaneously kicking the beam. These two correctors are generally the first two immediately upstream, but the user can re-select any desired one. Once this is done, $\alpha$ and $\beta$ are automatically loaded from the model. They can be overridden as well. The user will next choose an orbit size that corresponds to a desired emittance. The orbit size must be large enough to avoid noise, but small enough for the beam to remain in the beampipe. The user will then select how many turns, or times around the phase ellipse they desire, as well as how many data points on the ellipse to include.

Once all of these values are chosen, the user starts data acquisition. The program will first check to see if a system all-save has been performed recently, pausing to allow the user to perform one at that time if need be. All-saves are used at CEBAF so that we have knowledge of the conditions of the machine at the time of the test, and also provide a backup if something happens and the machine must be restored to a previous setup.

Next, the program will condition the corrector magnets by varying their values through the full range of settings that will be used for the procedure. This is done for two reasons. It provides a quick check that the chosen orbit size is not too large and that the beam will remain in the pipe. Additionally, preconditioning the iron core magnets reduces hysteretic asymmetries in the steering. They will maintain a level of reproducibility as the values of the corrector settings (BDL) are varied in both positive and negative directions. 


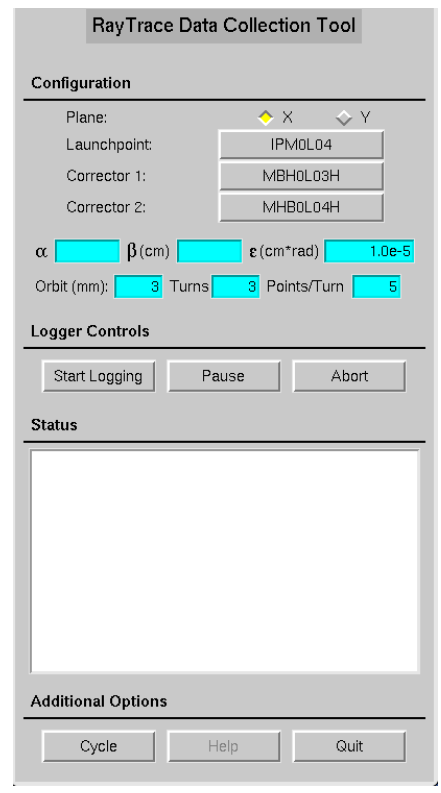

Figure 4.1: The rayTrace GUI

Once the magnets are conditioned, the two correctors are then kicked simultaneously as described before, and data is collected at every BPM downstream. As a failsafe and a time-saving feature for the analysis, data collection is automatically paused in the absence of beam.

\subsubsection{Model Twiss Parameter Acquisition}

At a time in close proximity to data acquisition, the model Twiss parameters must be acquired. Using a Perl script, I utilized the AHLA group's elegant toolkit to quickly grab the necessary values from the model and format it into Twiss tables, taking into account unit conversions.

Both the design model values and the values for the current setup (GOLD model) are acquired during this process.

\subsubsection{Analysis of Data: fitphase}

Yves Roblin's program, fitphase, is used in the analysis process. fitphase uses an algorithm that was originally designed for identifying ellipses in image data, but is also capable of utilizing raw statistical data. Iterating through each BPM, this program fits the model over a short range to determine the $x^{\prime}$ values. The user can choose the number of BPMs to use in this determination.

Once $x$ and $x^{\prime}$ are known, this trajectory data is used to compute $\epsilon, \alpha$, and $\beta$ at each BPM. This is then used to create plots containing the data points at the BPM, the ellipse fitted to these data points, and the design model phase ellipse at 


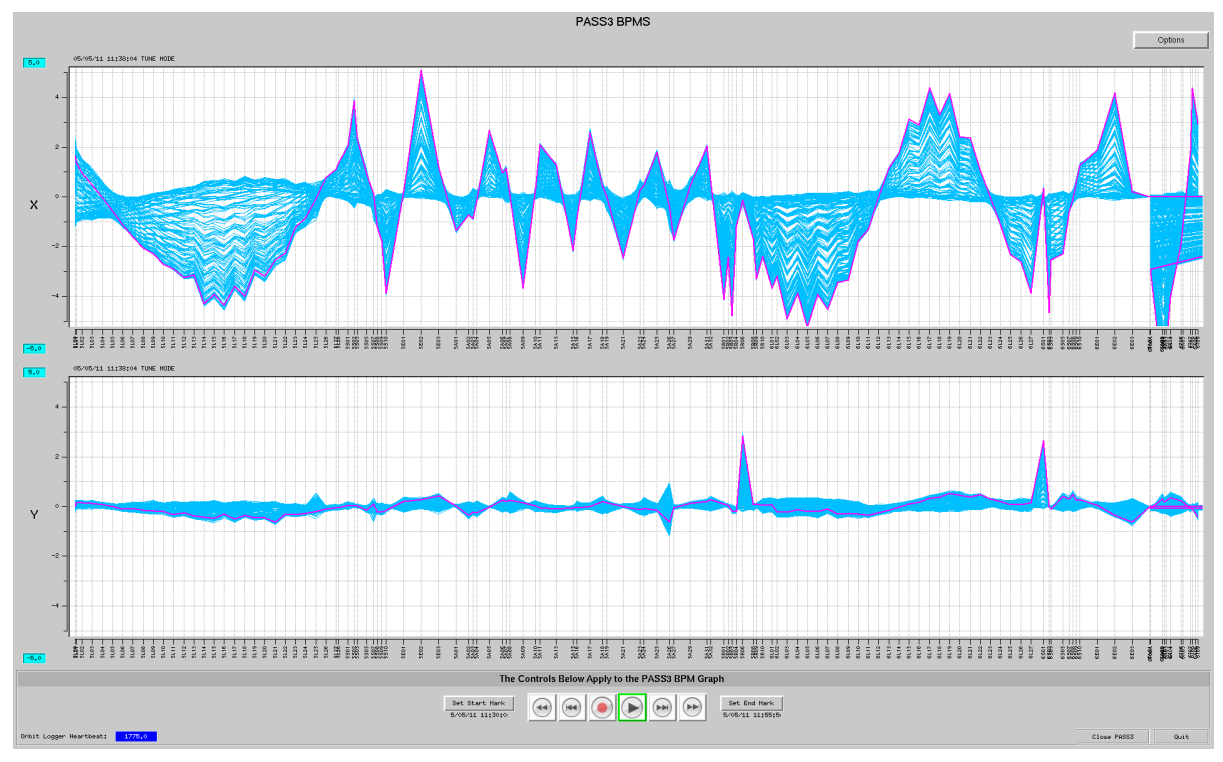

Figure 4.2: Overlaid Trajectories for 3 Pass CEBAF Machine

this location. This provides a fast, visual representation at each BPM and allows for quick assessment of the level of mismatch. Aberrations in the optics can also be seen in observing the overall shape made by the data points. Additionally, plots showing the variation of $\alpha$ and $\beta$ along the transverse direction are created. This is useful in understanding the global optical properties of the machine.

Although fitphase requires knowledge of the short range model to determine $x^{\prime}$, the data gathered still provides much information that can be found independently of the model.

For example, Figure 4.2 shows a plot of the overlaid trajectories found during the rayTrace procedure on a three-pass beam. Kicks were provided in the $\mathrm{x}$ plane. This plot provides a model-independent approximation of $\beta$, assuming that the emittance is known, as $\beta \approx \frac{\left(x_{\max }-x_{\min }\right)^{2}}{\epsilon}$. Also, the amount of crossplane coupling can be easily observed when looking at the plot for the y plane. This small amount of coupling is due to slightly rolled correctors. Since this is raw data, BPM noise is also included in this plot. Furthermore, it appears that some BPMs are reading the opposite plane, as indicated by the two large spikes in the $Y$-plane.

\subsubsection{Tuning the Machine}

To summarize, after running this procedure and analyzing the data, one will have data everywhere. The transverse angles are determined from the known local optics model. The Twiss parameters are generated for every point in the machine. One can discriminate between distributed and point errors and choose 


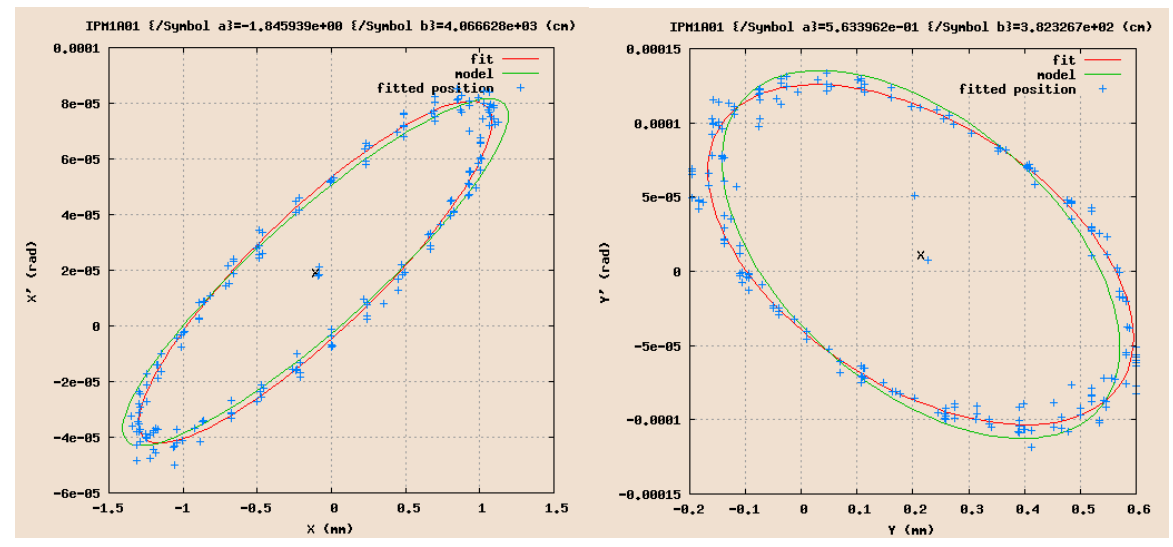

Figure 4.3: Injection Conditions in ARC 1

how to address the mismatch: globally or locally.

The "synthetic" beam is a surrogate for the real beam, acting as an exact linear model in the zero current limit. The emittance is magnified from the real beam, but the $\alpha$ and $\beta$ are the same. It also allows one to measure the phase advance of the machine, as data is collected in an orbit-by-orbit manner, giving the same kind of information one would gather from a numerical simulation.

\section{3 rayTrace in Action: An Example of Use}

Even early in the development, the rayTrace procedure proved useful several times in tuning the CEBAF machine. This section will discuss one example of its use.

In October of 2009, Hall A was experiencing high background count rates at its Compton polarimeter. The cause of this problem was unknown, and troubleshooting cost many man hours. The main problem that people were running into was the lack of adequate diagnostic tools in this region. Multiple quadrupole scans were run, but these results often showed asymmetric emittances. This left the operations crew with an iterative, guess-and-check method for tuning. Eventually, we were allowed to run rayTrace in the Hall A beamline to see if we could find any causes for the high background rate.

In order to gain both global, distributed information, as well as local information, we injected our synthetic beam at the beginning of ARC 1. Injecting so far upstream allowed us to see the progression of the synthetic beam through the machine so that we can check for any local problems that could contribute to distributed errors.

Choosing an emittance of $\epsilon=5 \times 10^{-6} \mathrm{~cm}$-rad, and using the design $\alpha$ and $\beta$, we provided 64 kicks at the two upstream correctors from the launchpoint, covering 2 turns around the phase ellipse (32 kicks/turn).

Looking at Figure 4.3, one can see the launch conditions in both the $\mathrm{X}$ and 


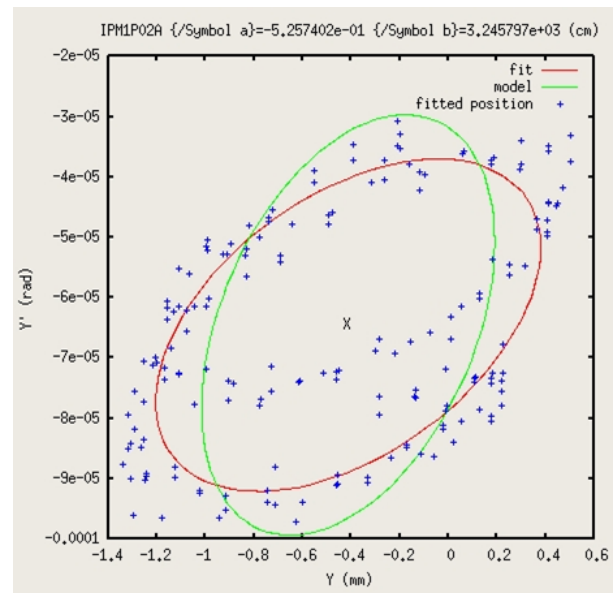

(a)

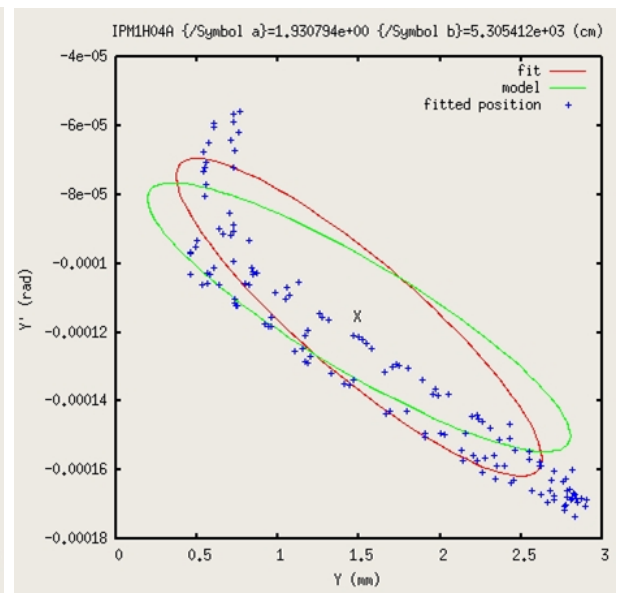

(b)

Figure 4.4: Y-Plane Aberration in Compton

Y planes. The injected orbit ellipse closely follows the design values. In these plots, the green ellipse represents the model phase ellipse, the blue points are the positions measured by the BPM at this point, and the red ellipse is fitted to these data points. $\alpha$ and $\beta$ are listed at the top of each plot as $\{/$ Symbol a $\}$ and $\{/$ Symbol b $\}$, respectively. One can also readily see what orbit size corresponds to the chosen emittance in each plane by looking at the abscissa, where it is over $2 \mathrm{~mm}$ for the horizontal plane, and approximately $0.75 \mathrm{~mm}$ in the vertical. The injected beam here is well-matched to the design orbit.

Hall A was receiving three-pass beam during this test. From the launchpoint, the beam travels 2.5 times around the accelerator, which translates to over 3 $\mathrm{km}$. We were able to track the beam's behavior over this distance, and found that by the time it reaches the Hall A line, it is mismatched from the design values. We also see that the emittances as measured damped approximately the same for the two planes from the launchpoint. This symmetric damping made the quadrupole scan data with asymmetric $\mathrm{X}$ and $\mathrm{Y}$ emittances appear suspect.

Once we reach the region of the Compton chicane, the apparent beam behavior becomes inconsistent with the large beamline clearances expected in the region. We began to see a strange aberration starting at BPM IPM1P02A (shown in Figure 4.4a), which is located at the beginning of the Compton chicane region. Initially, it was believed that the beam had drifted during the test, but further inspection led us to believe this was not the case. Downstream, the behavior grew worse (Figure 4.4b).

Recalling that beam had tripped off several times during the test, we looked at the beam loss archive and found a clue. At the times corresponding to vertical kicks, we experienced partial beam loss. This was due to the beam meeting with an aperture limitation, which we localized to the beginning of the 
Compton chicane. The unexpected behavior was due to either the beam being deflected, or the BPMs being unreliable due to the low current.

The data collection process took under fifteen minutes and gave 64 data points per plane at every BPM downstream from girder 1A01. After the analysis, two major problems were discovered. The first is that the beam entered the Hall A beamline mismatched from the model optics. The beam was then rematched using the normal protocols. The second is that there was partial beam loss somewhere in the Compton chicane region due to the beam being too near an aperture limitation in the vertical direction. The initial fix for this problem was to steer the beam away from the aperture limitation. Later, the hardware in the region was realigned and the synchrotron radiation in this region was baffled. Additionally, analysis showed that the procedure accounted for the appropriate amount of acceleration damping. The quad scans that had shown significant asymmetric emittances were found to be inaccurate, and the data that had been the most reasonable was then down-selected by the users so that corrections could be made with the most accurate data. 


\section{Part II}

\section{Noise Reduction and Uncertainty Analysis}




\section{Chapter 5}

\section{Singular Value Decomposition Analysis}

This chapter will discuss the analysis of rayTrace data using Singular Value Decomposition (SVD). The primary goal of performing SVD analysis on the data is to reduce the noise contribution in an effort to clean up the BPM signals. A secondary goal is to identify BPMs which have a significantly higher noise contribution, or are otherwise behaving in an unexpected or improper manner.

\subsection{Least Squares and Singular Value Decompo- sition}

One of the most powerful tools in our data analysis process is singular value decomposition (SVD), which is a variation on the traditional methods of least squares analysis. This section will discuss some of the basic concepts of both least squares analysis and SVD.

\subsubsection{Least Squares}

In a system where the number of descriptive equations outnumbers the number of unknowns in the equations, the system is said to be overdetermined. The term "least squares" derives from the minimization of the sum of all the squares of the errors when the equations are solved. In other words,

$$
S=\sum_{i=1}^{n} r_{i}^{2}
$$

would be a minimum in the optimum solution when fitting data. Here, the data set would consist of $n$ data points, $x_{i}$ would be the independent variable, $y_{i}$ would be the dependent (observed) variable, and $r_{i}$ is the residual. A residual is defined as the difference between the measured value of the dependent variable, 
and the value predicted by a given model, $f(x, \beta)$. In the model, there are $m$ adjustable parameters contained in the vector $\beta$. So, the residual is simply

$$
r_{i}=y_{i}-f\left(x_{i}, \beta\right) .
$$

The way to find the minimum of $S$ in Equation 5.1 is to set its gradient equal to zero. Because there are $m$ changeable parameters, there must be a matching number of gradient equations.

$$
\frac{\partial S}{\partial \beta_{j}}=2 \sum_{i} r_{i} \frac{\partial r_{i}}{\partial \beta_{j}}=0, j=1, \ldots, m
$$

There are two types of least squares analysis: linear and non-linear. A linear least squares (LLSQ) model function will consist of a linear combination of parameters, such as $f=X_{i 1} \beta_{1}+X_{i 2} \beta_{2}+\ldots$. A non-linear least squares (NLLSQ) model function will have the model function appear in non-linear

functions, such as $f=e^{\beta x}$. Another way to think of this is that if $\frac{\partial f}{\partial \beta_{j}}$ is either constant or depends only on the independent variables, the model is linear. Otherwise, the model is non-linear, and to find a solution one must choose initial values for the parameters, and the process is usually iterative in nature, terminating once a specific criterion of convergence is satisfied. Additionally, LLSQ has a unique solution, given the direct methods of finding the solutions. NLLSQ may have multiple solutions, as there may be multiple local minima.

\subsubsection{Singular Value Decomposition $[1,23,28]$}

The purpose of Singular Value Decomposition (SVD) is to reduce a set of data containing both correlated and uncorrelated values to a set of data which contains significantly fewer uncorrelated values while maintaining the majority of the variability present in the original set of data. In order to use SVD, one must be aware that the data must contain anomalies, and it should be detrended so that only the absolute changes in values are shown. Without detrending, contributions which are uncorrelated to the dominant singular values will be magnified. Detrending can be accomplished by subtracting the average value of a data set from each data point.

The most basic definition of Singular Value Decomposition is that it is a factorization of a matrix. It is defined mathematically by

$$
M=U \Sigma V^{*}
$$

where $M$ is a $m \times n$ matrix, $U$ in a $m \times m$ unitary matrix, $\Sigma$ is a $m \times n$ diagonal matrix containing non-negative real numbers for the diagonal terms, and $V^{*}$, or the conjugate transpose of $V$ (sometimes written as $V^{\dagger}$ or $V^{T}$, depending on the real or imaginary nature of the data), is an $n \times n$ unitary matrix. Additionally, the diagonal values in $\Sigma$ are the singular values of $M$. The left singular vectors of the $M$ matrix are the $m$ columns of the $U$ matrix, and are the eigenvectors of $M M^{*}$. The $n$ columns of the $V$ matrix are the right singular vectors, and 


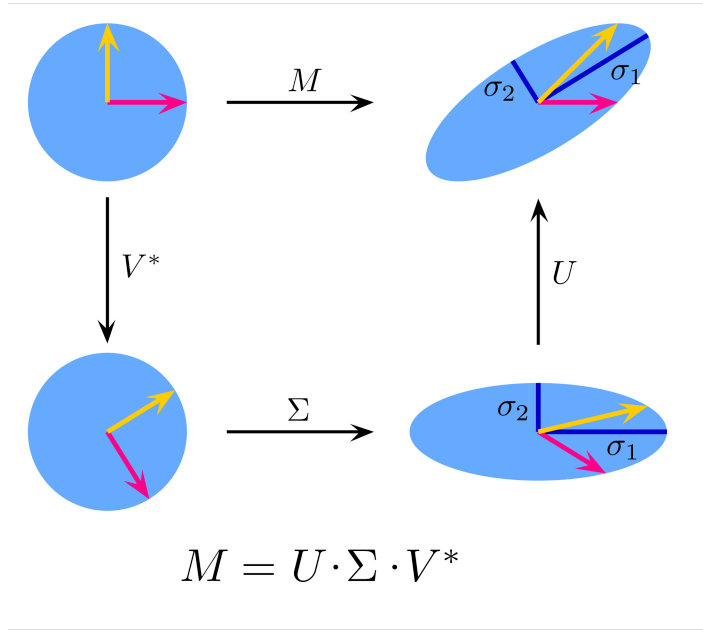

Figure 5.1: SVD Flow Chart

they are the eigenvectors of $M^{*} M$. Taking the square roots of the non-zero eigenvalues of $M M^{*}$ or $M^{*} M$ gives the singular values in $\Sigma$.

Each of the matrices in Equation 5.4 act to decompose the matrix $M$ into three simple transformations. For example, in Figure 5.1, $M$ represents the real shearing matrix in $2 \mathrm{D}$ of a unit circle. When applied to the circle, the result is an ellipse. This same outcome can be accomplished in a series of steps. The first step is applying

$$
V^{*}=\left[\begin{array}{ll}
V_{11}^{*} & V_{12}^{*} \\
V_{21}^{*} & V_{22}^{*}
\end{array}\right],
$$

which rotates the circle clockwise. This step can further be broken down by column in $V^{*}$, as the first column shears the circle horizontally, and then the second column shears the ellipse vertically. The end result is a rotated unit circle.

Next,

$$
\Sigma=\left[\begin{array}{ll}
\Sigma_{11} & \Sigma_{12} \\
\Sigma_{21} & \Sigma_{22}
\end{array}\right]
$$

is applied, which provides scaling along the coordinate axes. Here, $\Sigma_{12}$ and $\Sigma_{21}$ are both zero. The first column elongates the circle in the horizontal direction, and the second column adjusts the height. This results in an upright ellipse, where the semi-major $\left(\sigma_{1}\right)$ and semi-minor $\left(\sigma_{2}\right)$ axes correspond to the singular values along the diagonal of $\Sigma$.

Finally, a second rotation is provided by the matrix

$$
U=\left[\begin{array}{ll}
U_{11} & U_{12} \\
U_{21} & U_{22}
\end{array}\right] .
$$

Here, the first column will shear/rotate the ellipse horizontally, and the second column will do so vertically. The end result will be a rotated ellipse. The end 
result of all three steps will be equivalent to the transformation accomplished by matrix $M$.

In order to find the SVD of a matrix $A$, several steps must be taken. I will describe these steps presently, the first of which is computing the singular values of $A$. First (I will change to the $X^{T}$ nomenclature), the transpose of $A$ is found, $A^{T}$. $A$ and $A^{T}$ are then multiplied together. $A A^{T}$ would be a left multiplication, and the resulting matrix would be the left matrix. $A^{T} A$ would be a right multiplication, and the resulting matrix would be a right matrix. For each of these matrices, one would find the eigenvalues by setting $\operatorname{det}\left[A^{T} A-\lambda I\right]=0$ and solving for $\lambda$. Once found, place them in descending order in an absolute sense. Taking the square root of these eigenvalues gives the singular values, which are then placed along the diagonal of the $\Sigma$ matrix, with all off-diagonal terms equal to zero.

If $A$ is a rectangular, $m \times n$ matrix, then the only useful singular values are those that correspond to the smaller value of $m$ or $n$. For example, if $m>n$, one can think of the SVD as mapping $n$-space onto $m$-space, with any of the singular values that correspond to columns greater than $n$ are unnecessary. In short, if:

- $m=n$, all of the singular values will be positive.

- $m<n, \Sigma$ will be a $m \times n$ diagonal matrix with all of the column elements greater than $m$ equaling zero.

- $m>n, \Sigma$ will be a $n \times n$ diagonal matrix, giving more equations than unknowns. In this case, the solution to the SVD will be a least squares solution.

The next step in finding the SVD of matrix $A$ is to compute the right eigenvectors, which will be found from the right matrix, $A^{T} A$. Having already found the eigenvalues and placed them in descending order, one can simply go through each of them and find the corresponding eigenvector. Simply plug the values into the equation $\left(A^{T} A-\lambda I\right) \vec{X}=0$, where $\lambda$ is the eigenvalue, and $\vec{X}$ is the eigenvector. Solving each equation for the the vector terms with respect to each other, and normalizing by dividing each term by the length $\left(L=\sqrt{\sum_{i} x_{i}^{2}}\right)$ gives the eigenvector corresponding to that eigenvalue. One would repeat this for each eigenvalue in descending order. Paying careful attention to the order in which these are found so that the matrices are organized in a simpler way, 
the eigenvectors are then placed into the columns of the $V$ matrix. So, if

$$
\begin{aligned}
\vec{X}_{1} & =\left[\begin{array}{l}
x_{1,1} \\
x_{1,2} \\
x_{1,3}
\end{array}\right], \\
\vec{X}_{2} & =\left[\begin{array}{l}
x_{2,1} \\
x_{2,2} \\
x_{2,3}
\end{array}\right], \text { and } \\
\vec{X}_{3} & =\left[\begin{array}{l}
x_{3,1} \\
x_{3,2} \\
x_{3,3}
\end{array}\right], \text { then } \\
V & =\left[\begin{array}{lll}
x_{1,1} & x_{2,1} & x_{3,1} \\
x_{1,2} & x_{2,2} & x_{3,2} \\
x_{1,3} & x_{2,3} & x_{3,3}
\end{array}\right] .
\end{aligned}
$$

One would then take the transpose of this matrix for use in the SVD.

This process could be repeated for the left eigenvectors, substituting $A A^{T}$ for $A^{T} A$ and doing the math accordingly, placing the eigenvectors into the columns of matrix $U$. This can be useful in finding high-order, term-term co-occurrence patterns. However, this process can be time consuming and computationally time consuming. There exists a shortcut to find the left eigenvectors. Starting with the definition of SVD, and the fact that $\Sigma$ and $V$ are already known, one can show that:

$$
\begin{aligned}
A & =U \Sigma V^{T} \\
A V & =U \Sigma V^{T} V=U \Sigma \\
A V \Sigma^{-1} & =U \Sigma \Sigma^{-1}=U I \\
U & =A V \Sigma^{-1} .
\end{aligned}
$$

Now one has all of the required pieces in order to perform SVD on the matrix, $A$. By substituting the above steps into the original SVD equation, Equation 5.5 , one finds that the values on the right side of the equation will nearly equal those on the left.

Additionally, one can perform a reduced SVD, which will still give an approximation of the original matrix, but will truncate the matrices in such a manner that noisy dimensions will be reduced and the effects of the largest $k$ singular values can be exposed. To do this, simply keep the first $k$ columns of $U$, the first $k$ rows of $V^{T}$, and the first $k$ singular values.

In summary, the steps required for calculating the SVD of a matrix $A$ are as follows:

1. Find $A^{T}, A^{T} A$, and $A A^{T}$.

2. Find the eigenvalues of $A^{T} A$ and $A A^{T}$ and sort them in descending order in the absolute sense. Take the square roots of these to find the singular values. 
3. Make a diagonal matrix, $\Sigma$, with the singular values along the diagonal. Find the inverse of this matrix, $\Sigma^{-1}$.

4. Use the eigenvalues from Step 2 to compute the eigenvectors for $A^{T} A$. Place these values along the columns of $V$, taking care to keep them in order. Compute $V^{T}$.

5. Either repeat Step 4 for $A A^{T}$, or use the shortcut equation, Equation 5.6, to find $U$.

6. If a reduced SVD is desired, truncate the matrices appropriately to reduce noise.

\subsection{SVD Noise Reduction [18, 23, 28, 29]}

As described in the dissertation of C.X. Wang [23], SVD is a powerful tool for noise-reduction of data. Applying some of the principles described in this work, I performed noise-reduction analysis on the raw BPM data acquired through the rayTrace procedure, as described in 4.2.1.

\subsubsection{Raw rayTrace Data}

When rayTrace is performed, position information at every BPM in CEBAF is recorded simultaneously. As the procedure varies two correctors simultaneously ("kicking" them iteratively every few seconds) to trace the boundary of a phase ellipse, the BPM system reports position data at every location several times per kick, which is recorded. This data is formatted in such a manner that every BPM has its own column of position data. Each row of data contains the reported position of the beam at each BPM location at a specific time. Figure 5.2 shows the format of the collected data. Additionally, when no beam is present in the machine, this is also reported in the data, and must be removed during the analysis process.

With the data formatted in this manner, there are two bases that can be used to describe it: a spatial (or position) basis corresponding to the columns of BPMs, which are ordered according to longitudinal position in the machine, and a temporal basis corresponding to the rows of time-dependent measured positions. When analyzing a segment of CEBAF, such as a single linac, only the BPMs found within this segment are included. This generally results in a $m \times n$ matrix where $m>n$, i.e. a tall, thin matrix.

In looking at a plot of the raw BPM data from the X-plane of the first arc (Figure 5.3), it can be seen that some data pre-processing must be performed. Each signal on this plot is a different BPM, represented by the various colors. Any signals which appear nearly flat in this plot may, but do not necessarily, represent a BPM that has its wires switched in such a manner that the $\mathrm{X}$ and $\mathrm{Y}$ planes are interchanged. These columns of data must be swapped (and the problem reported to the Operations group). Most importantly though, the data 


\begin{tabular}{|c|c|c|c|c|c|c|c|c|c|c|c|}
\hline $\begin{array}{c}\text { BPM } \\
1\end{array}$ & $\begin{array}{c}\text { BPM } \\
2\end{array}$ & $\begin{array}{c}\text { BPM } \\
3\end{array}$ & $\begin{array}{c}\text { BPM } \\
4\end{array}$ & $\begin{array}{c}\text { BPM } \\
5\end{array}$ & $\begin{array}{c}\text { BPM } \\
6\end{array}$ & $\begin{array}{c}\text { BPM } \\
7\end{array}$ & $\begin{array}{c}\text { BPM } \\
8\end{array}$ & $\begin{array}{c}\text { BPM } \\
9\end{array}$ & $\begin{array}{c}\text { BPM } \\
10\end{array}$ & $\cdots$ & $\cdots$ \\
\hline $\begin{array}{c}\text { Data } \\
1\end{array}$ & $\begin{array}{c}\text { Data } \\
1\end{array}$ & $\begin{array}{c}\text { Data } \\
1\end{array}$ & $\begin{array}{c}\text { Data } \\
1\end{array}$ & $\begin{array}{c}\text { Data } \\
1\end{array}$ & $\begin{array}{c}\text { Data } \\
1\end{array}$ & $\begin{array}{c}\text { Data } \\
1\end{array}$ & $\begin{array}{c}\text { Data } \\
1\end{array}$ & $\begin{array}{c}\text { Data } \\
1\end{array}$ & $\begin{array}{c}\text { Data } \\
1\end{array}$ & $\cdots$ & $\cdots$ \\
\hline $\begin{array}{c}\text { Data } \\
2\end{array}$ & $\begin{array}{c}\text { Data } \\
2\end{array}$ & $\begin{array}{c}\text { Data } \\
2\end{array}$ & $\begin{array}{c}\text { Data } \\
2\end{array}$ & $\begin{array}{c}\text { Data } \\
2\end{array}$ & $\begin{array}{c}\text { Data } \\
2\end{array}$ & $\begin{array}{c}\text { Data } \\
2\end{array}$ & $\begin{array}{c}\text { Data } \\
2\end{array}$ & $\begin{array}{c}\text { Data } \\
2\end{array}$ & $\begin{array}{c}\text { Data } \\
2\end{array}$ & $\cdots$ & $\cdots$ \\
\hline $\begin{array}{c}\text { Data } \\
3\end{array}$ & $\begin{array}{c}\text { Data } \\
3\end{array}$ & $\begin{array}{c}\text { Data } \\
3\end{array}$ & $\begin{array}{c}\text { Data } \\
3\end{array}$ & $\begin{array}{c}\text { Data } \\
3\end{array}$ & $\begin{array}{c}\text { Data } \\
3\end{array}$ & $\begin{array}{c}\text { Data } \\
3\end{array}$ & $\begin{array}{c}\text { Data } \\
3\end{array}$ & $\begin{array}{c}\text { Data } \\
3\end{array}$ & $\begin{array}{c}\text { Data } \\
3\end{array}$ & $\cdots$ & $\cdots$ \\
\hline $\begin{array}{c}\text { No } \\
\text { Beam }\end{array}$ & $\begin{array}{c}\text { No } \\
\text { Beam }\end{array}$ & $\begin{array}{c}\text { No } \\
\text { Beam }\end{array}$ & $\begin{array}{c}\text { No } \\
\text { Beam }\end{array}$ & $\begin{array}{c}\text { No } \\
\text { Beam }\end{array}$ & $\begin{array}{c}\text { No } \\
\text { Beam }\end{array}$ & $\begin{array}{c}\text { No } \\
\text { Beam }\end{array}$ & $\begin{array}{c}\text { No } \\
\text { Beam }\end{array}$ & $\begin{array}{c}\text { No } \\
\text { Beam }\end{array}$ & $\begin{array}{c}\text { No } \\
\text { Beam }\end{array}$ & $\cdots$ & $\cdots$ \\
\hline $\begin{array}{c}\text { No } \\
\text { Beam }\end{array}$ & $\begin{array}{c}\text { No } \\
\text { Beam }\end{array}$ & $\begin{array}{c}\text { No } \\
\text { Beam }\end{array}$ & $\begin{array}{c}\text { No } \\
\text { Beam }\end{array}$ & $\begin{array}{c}\text { No } \\
\text { Beam }\end{array}$ & $\begin{array}{c}\text { No } \\
\text { Beam }\end{array}$ & $\begin{array}{c}\text { No } \\
\text { Beam }\end{array}$ & $\begin{array}{c}\text { No } \\
\text { Beam }\end{array}$ & $\begin{array}{c}\text { No } \\
\text { Beam }\end{array}$ & $\begin{array}{c}\text { No } \\
\text { Beam }\end{array}$ & $\cdots$ & •• \\
\hline $\begin{array}{c}\text { Data } \\
4\end{array}$ & $\begin{array}{c}\text { Data } \\
4\end{array}$ & $\begin{array}{c}\text { Data } \\
4\end{array}$ & $\begin{array}{c}\text { Data } \\
4\end{array}$ & $\begin{array}{c}\text { Data } \\
4\end{array}$ & $\begin{array}{c}\text { Data } \\
4\end{array}$ & $\begin{array}{c}\text { Data } \\
4\end{array}$ & $\begin{array}{c}\text { Data } \\
4\end{array}$ & $\begin{array}{c}\text { Data } \\
4\end{array}$ & $\begin{array}{c}\text { Data } \\
4\end{array}$ & $\cdots$ & $\cdots$ \\
\hline : & : & : & : & : & : & : & : & : & : & : & : \\
\hline
\end{tabular}

Figure 5.2: Format of Collected BPM Data

must be detrended so that correlated contributions due to BPM offsets are not included in the analysis. This is done by subtracting the average value of each column of data from the each data point in that column.

This data pre-processing is done in MATLAB. To do this, you first load the data file you plan to analyze: load datafile.dat. You then find the size of the data matrix: $[n, p]=$ size (datafile); . Next you compute the mean of each column: mu=mean (datafile);. Next, you create a matrix of the mean values by repeating this last step for the number of rows: MeanMat=repmat $(m u, n, 1)$;. Finally, you subtract the column mean from each element, yielding a detrended data matrix: $\mathrm{T}=$ datafile-MeanMat; .

In order to select only the data for the segment to be analyzed, MATLAB scripts are used to find and order the correct BPM columns. For example, the script to select ARC1 BPMs is:

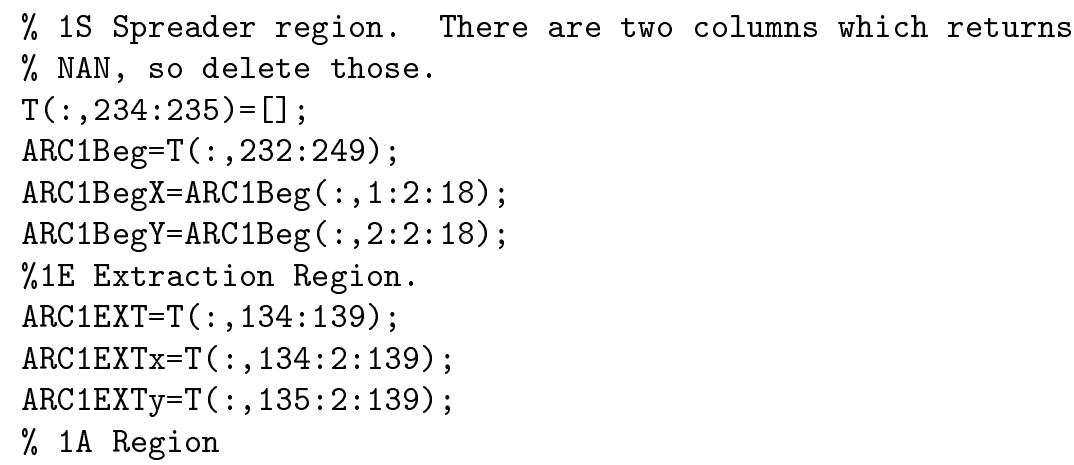




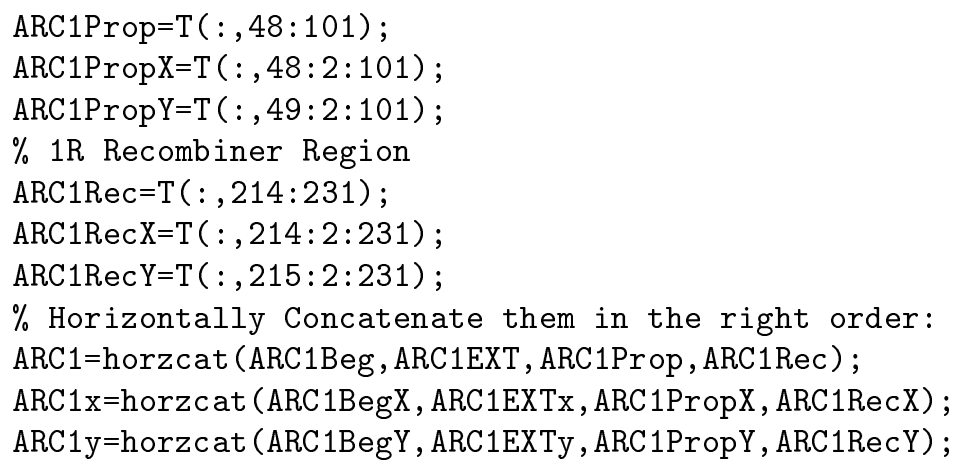

This script selects the columns that correspond to BPMs in the spreader, extraction, arc, and recombiner regions of CEBAF's Arc 1, and creates matrices for the whole Arc, as well as each plane separately. So, after creating the detrended $\mathrm{T}$ data matrix, the script takes $\mathrm{T}$ and specifies which columns to use for analysis.

The detrended and pre-processed data will look like Figure 5.4. The average value of each signal is now zero (to within machine precision).

\subsubsection{SVD Procedure}

Once the data has been properly detrended and preprocessed, SVD is performed using MATLAB's built-in function. For example, to perform SVD on the X-plane of $\operatorname{Arc} 1:[\mathrm{U}, \mathrm{S}, \mathrm{V}]=\operatorname{svd}(\operatorname{ARC} 1 \mathrm{x})$;. Here, $U$ is the temporal basis matrix, $V$ is the spatial basis matrix, and $S$ is the diagonal matrix containing the singular values ordered by magnitude.

Plotting the singular values (diagonal values of the $S$ matrix) gives one a visual guide for cutting noise terms. The largest singular values correspond to the bases which have the most dominant correlated contributions to the total signal. Figure 5.5 is a plot of the singular values for the horizontal plane of Arc 1 without detrending the data. Notice the two dominant singular values near the top of the plot. When looking at Figure 5.6, which is a plot of the singular values for the same region and orientation with detrended data, the second of these large singular values is no longer present. With the detrended data, correlations related to BPM offsets and measurement biases are removed, thus reducing the degrees of freedom. The detrended data has one very dominant singular value, as well as at least one smaller which may or may not contribute in a significant way. In order to quantify the significance of the smaller singular values, one must first look at the corresponding spatial and temporal basis vectors.

Looking first at the temporal basis vectors, the first 16 of which are plotted in Figure 5.7, one sees that those corresponding to the two largest singular values vary sinusoidally over time, which is expected due to the nature of this procedure. The third and fourth vectors appear to be noise contributions, but further investigation is required. 


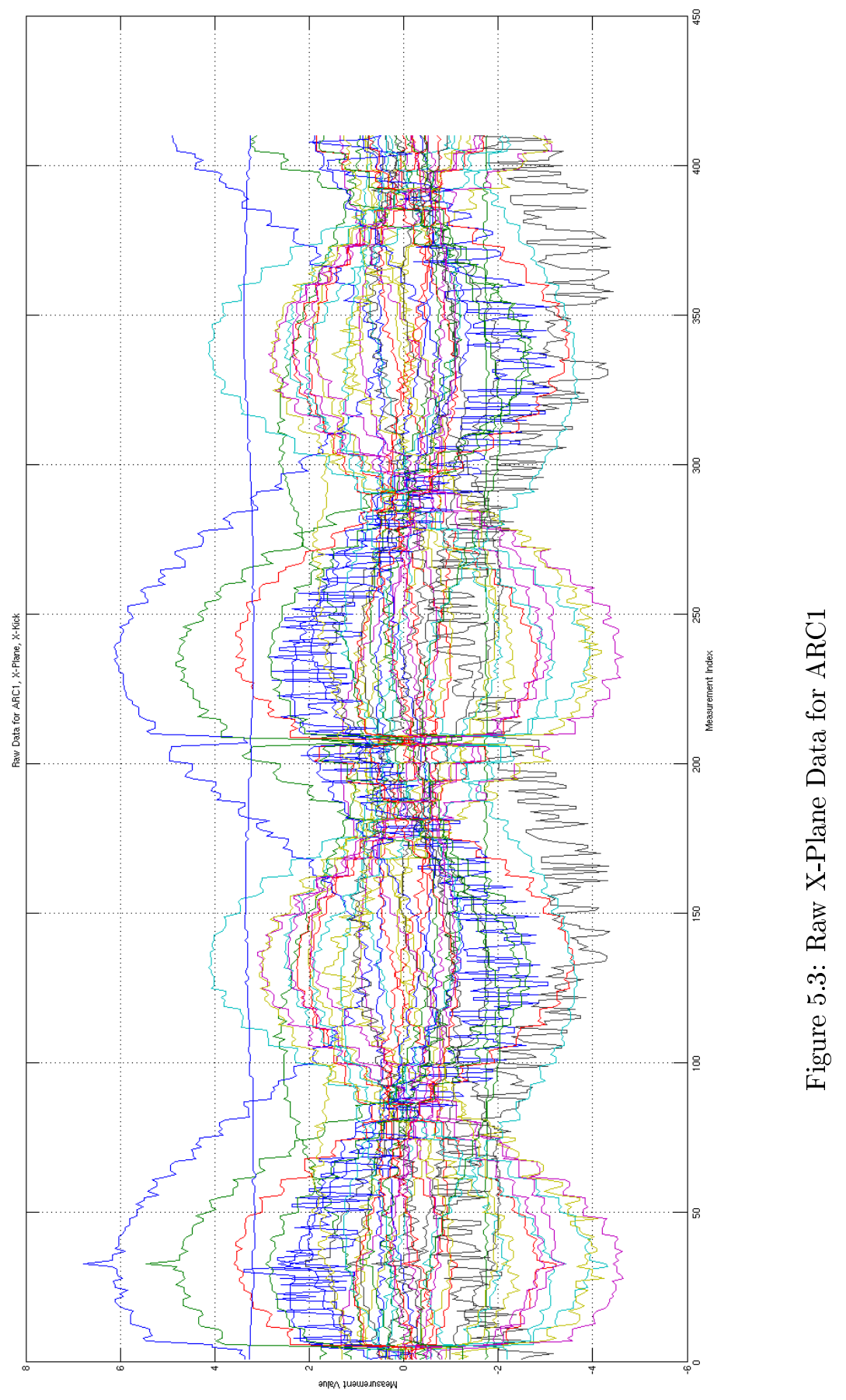




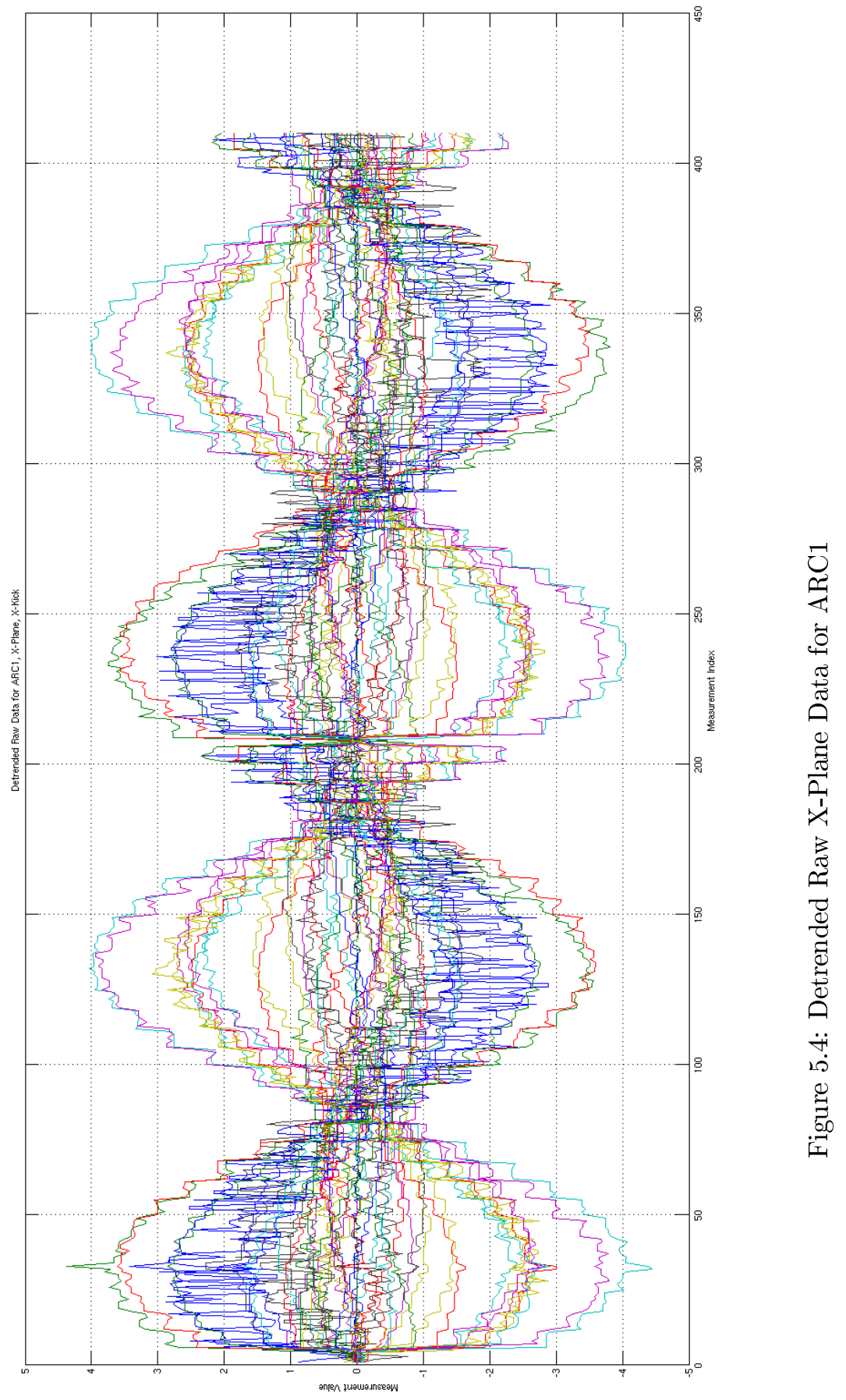




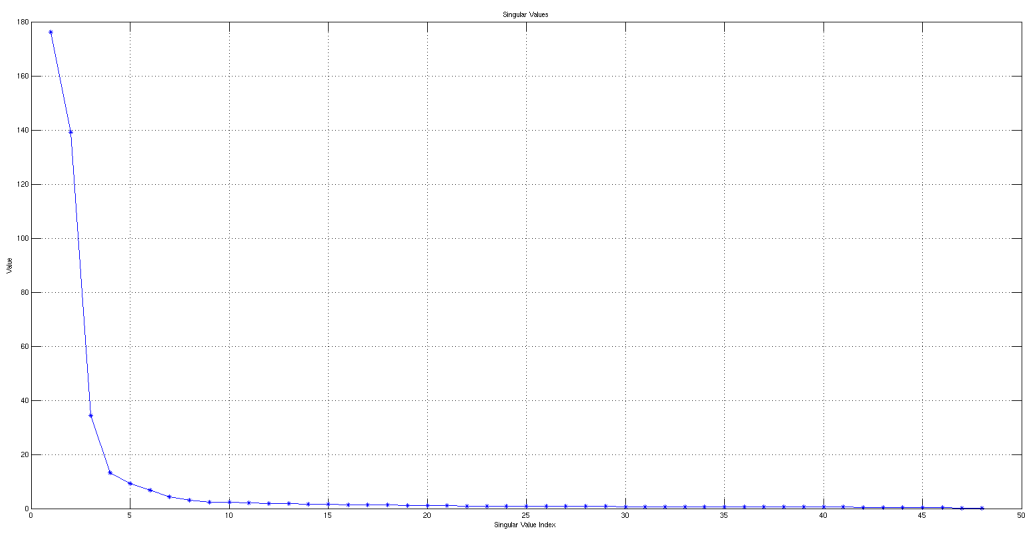

Figure 5.5: Singular Values for Non-Detrended Data: Arc1, X-Plane, X-Kick. Axes are Singular Value (0-180) vs. Singular Value Index (0-50)

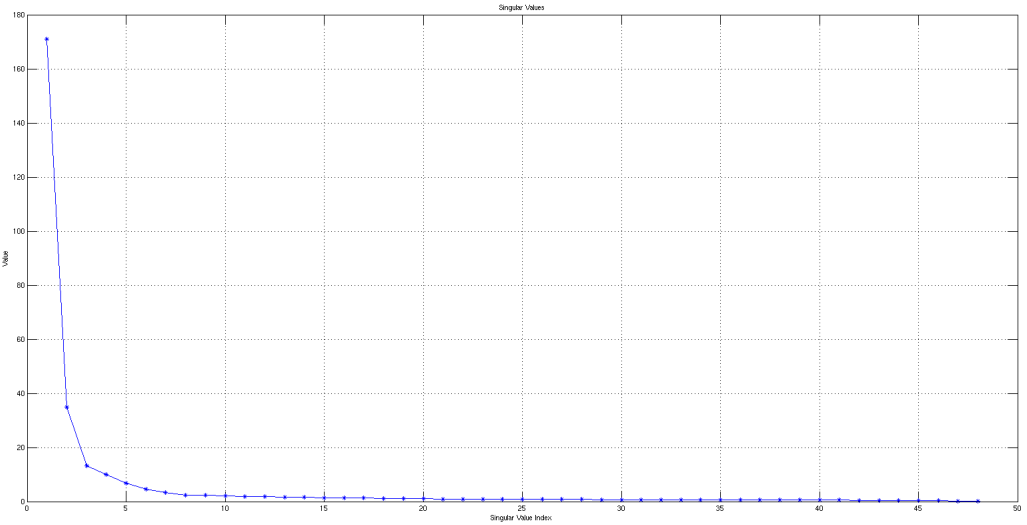

Figure 5.6: Singular Values for Detrended Data: Arc 1, X-Plane, X-Kick. Axes are Singular Value (0-180) vs. Singular Value Index (0-50) 

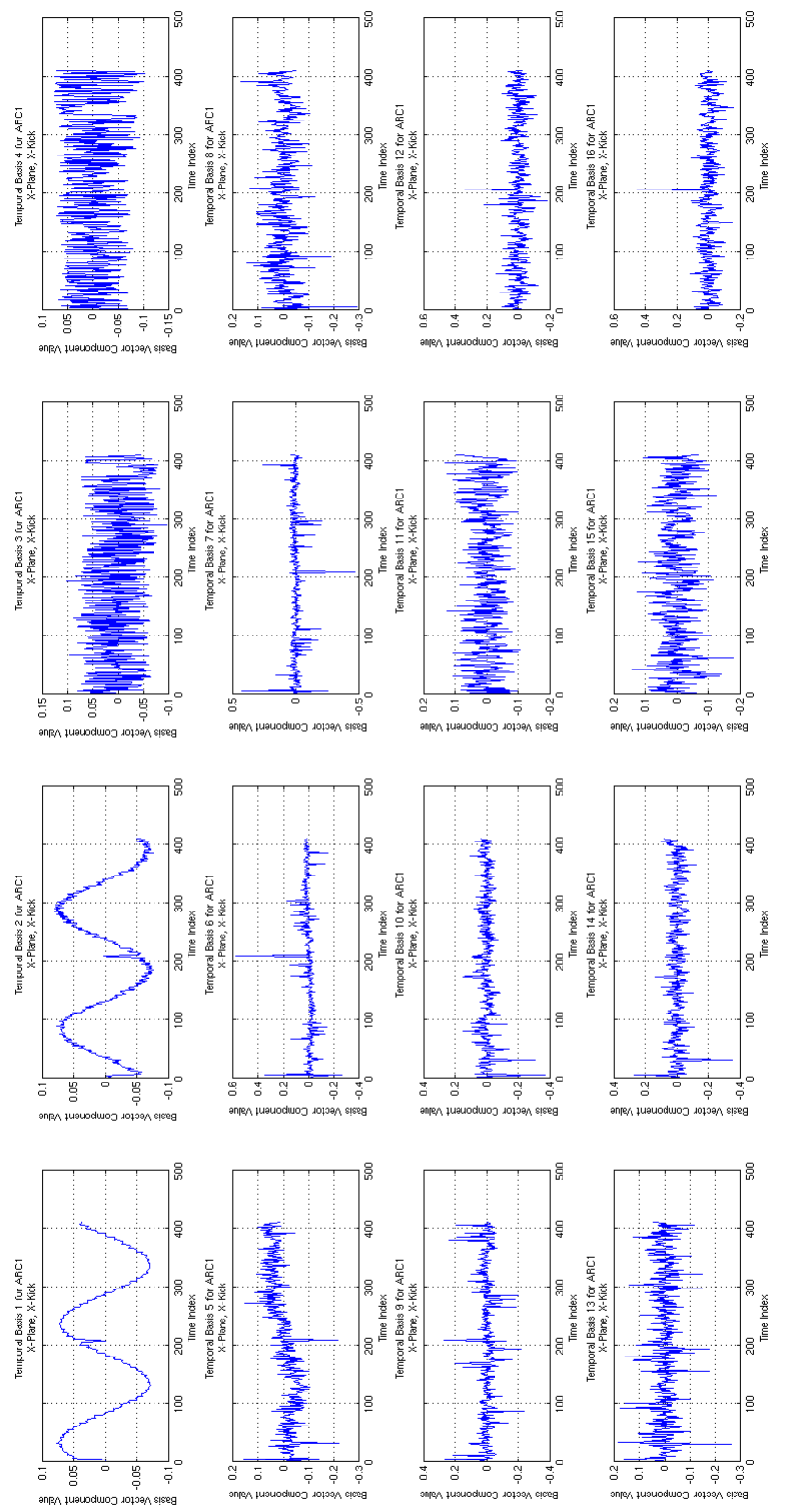

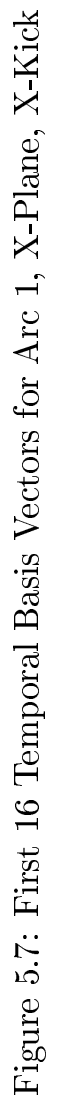


Looking next at the spatial basis vectors, plotted in Figure 5.8, it can be seen that the first two follow a pattern with some level of periodicity, but that the third and fourth vectors are large spikes at single locations. Since these vectors are normalized, and the spikes for the vectors corresponding to the third and fourth singular values near unity, this means that nearly all of the contribution from this vector comes from a single BPM. Looking back at Figure 5.7, it appears that these BPMs are reading noise. To be sure, a Fast Fourier Transform (FFT) was performed on the temporal basis vector. Figure 5.9 shows plots of the spatial and temporal basis vectors corresponding to the largest four singular values, as well as periodograms (from the FFT of the temporal basis vectors). The FFT analysis shows that the contributions of the third and fourth temporal basis vectors are, indeed, noise, and that only the vectors corresponding to the largest two singular values contribute significantly to the correlated responses in the data. All those vectors corresponding to smaller singular values are either due to noise, or do not significantly contribute to the correlated responses and can be cut.

To cut the singular values that correspond to noise and other uncorrelated signal contributions, one simply sets these values to zero. This is done very simply in MATLAB with the command $\mathrm{S}(\mathrm{S}<\mathrm{x})=0$; , where $x$ is the value of the lowest singular value that you wish to keep. After cutting the singular values, one can then reconstruct the original data without the noise and uncorrelated terms by reversing the process; CleanData=U*S*V';. Here, $U$ and $V$ are the original eigenvector matrices, and $S$ is a truncated diagonal matrix, containing only the most dominant singular values, which occupy the first few elements of this matrix. All other terms are zero. This creates a data matrix of the same size as the original, but filtered by enforcing the correlations among BPMs associated with the retained eigenvectors. Figures 5.10 and 5.11 show the before-and-after noise reduction for all of the BPMs in Arc 1 for the X-plane.

To further demonstrate the ability of this method to reduce the noise in the system, one can view the before-and-after signals of the noisiest BPM in Arc 1 in Figure 5.12. The blue signal is the detrended, raw signal for IPM1A23. The red signal is the noise-reduced signal for the same BPM. Notice that there is a fiducial point where the beam centroid is deliberately steered back to the zero location prior to the second cycle of the data collection. This method of noise reduction preserves this point. 

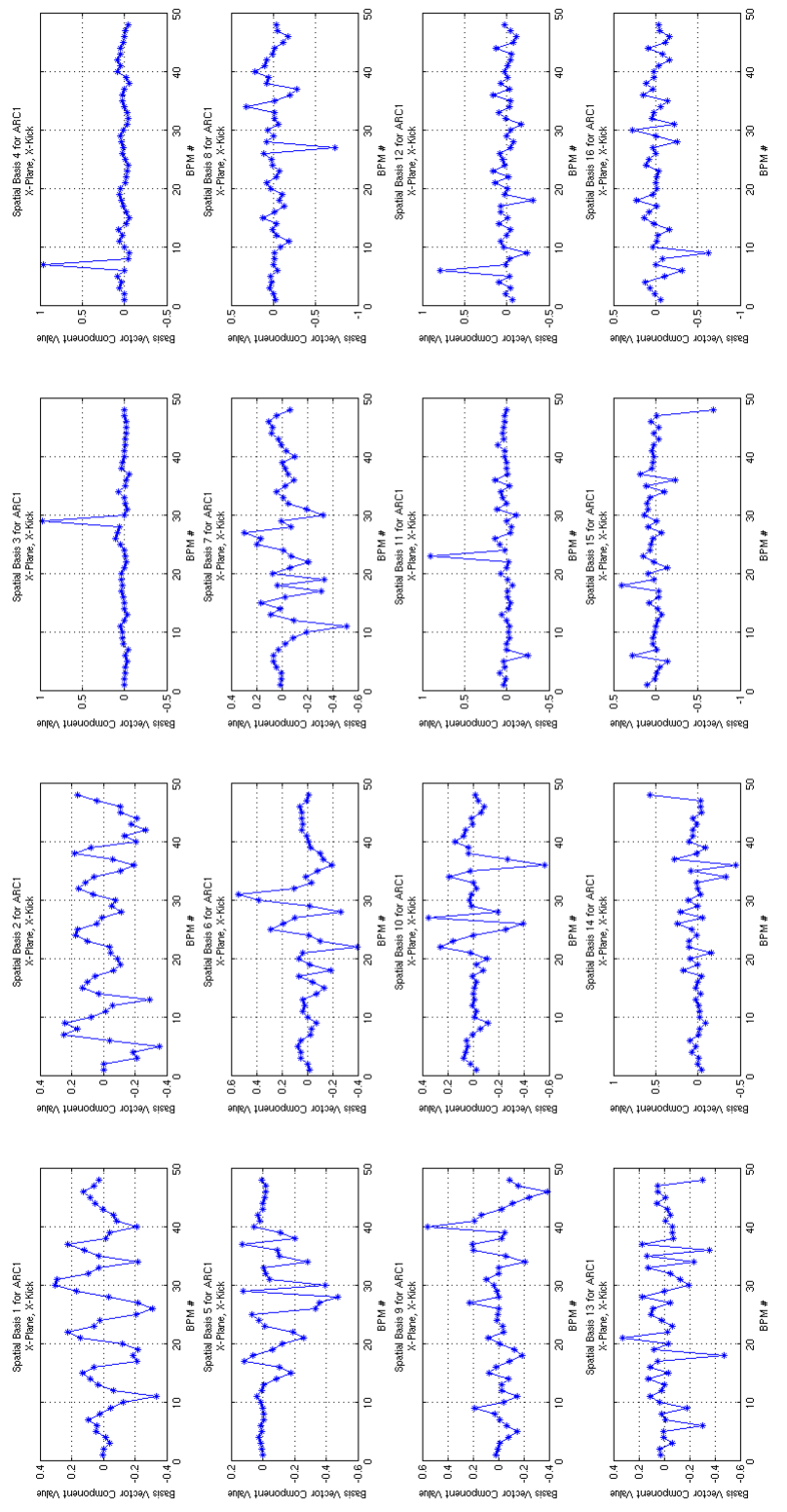

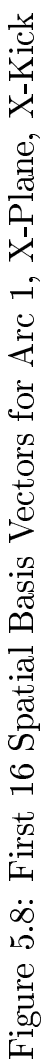



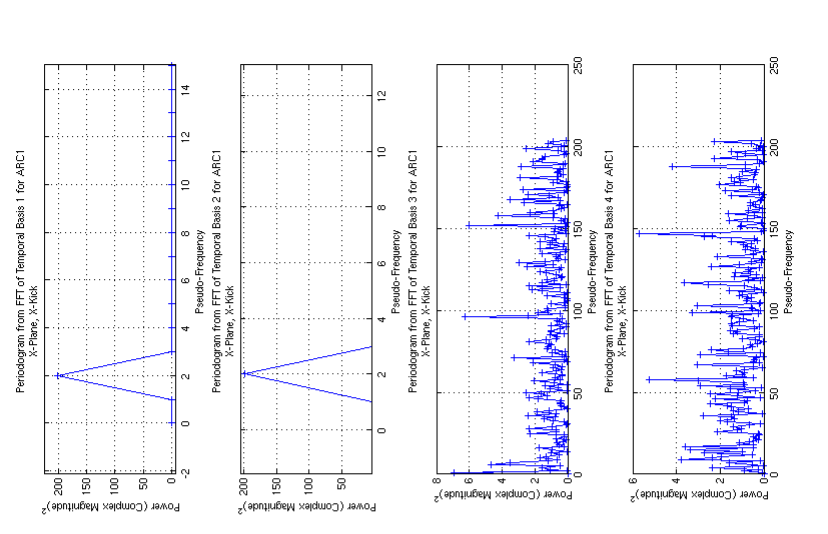

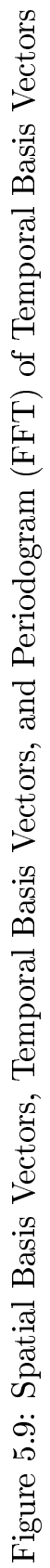




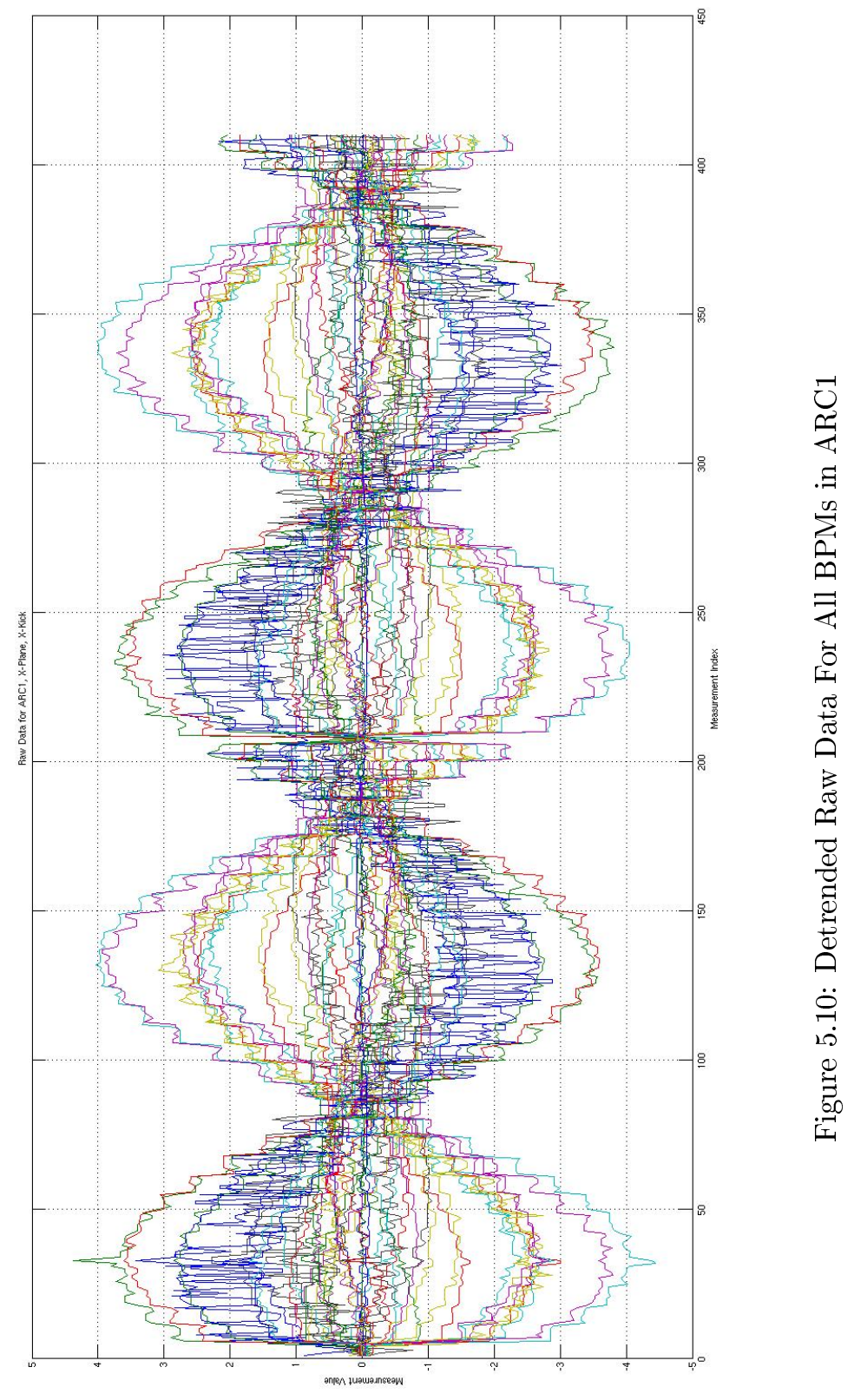




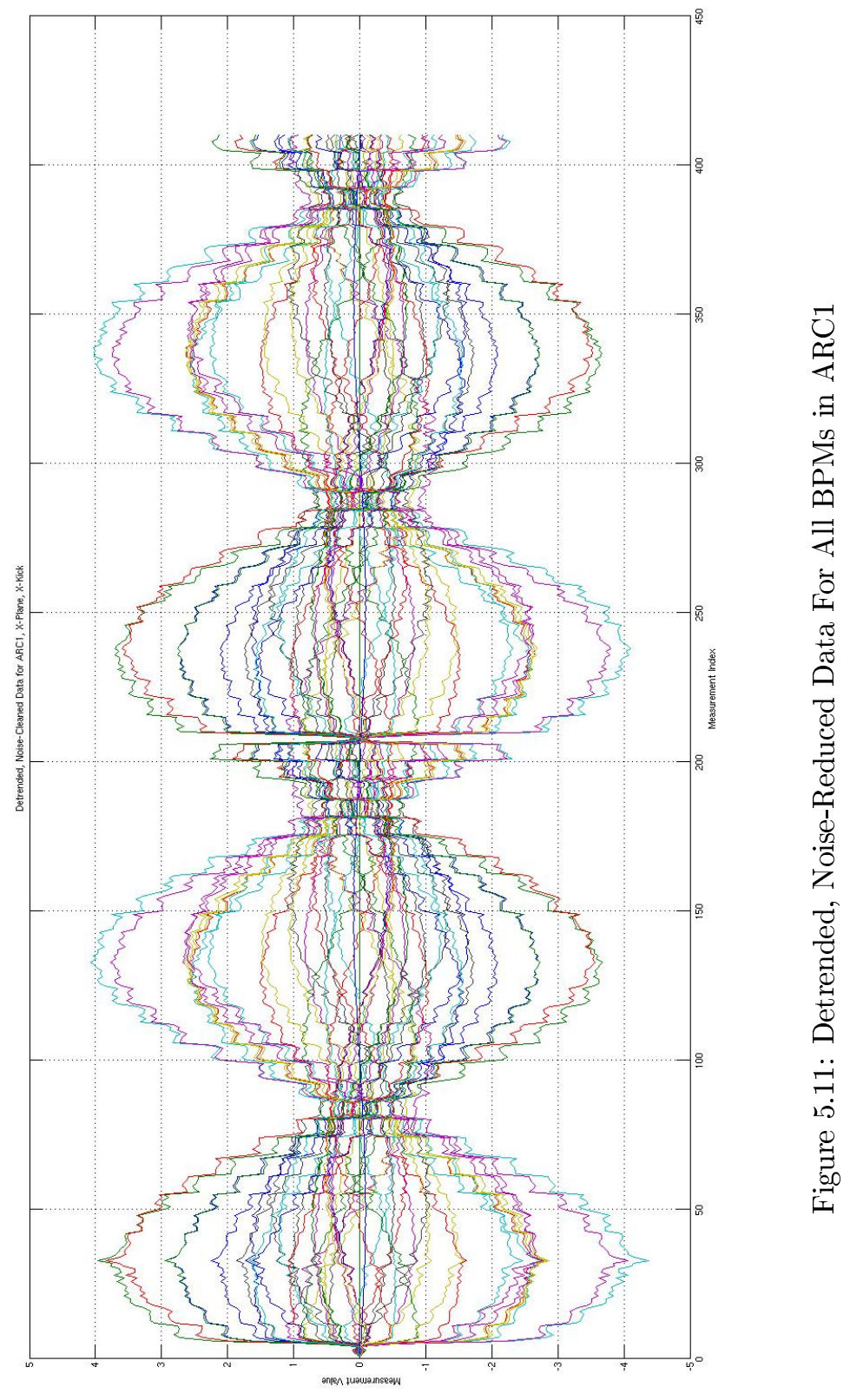




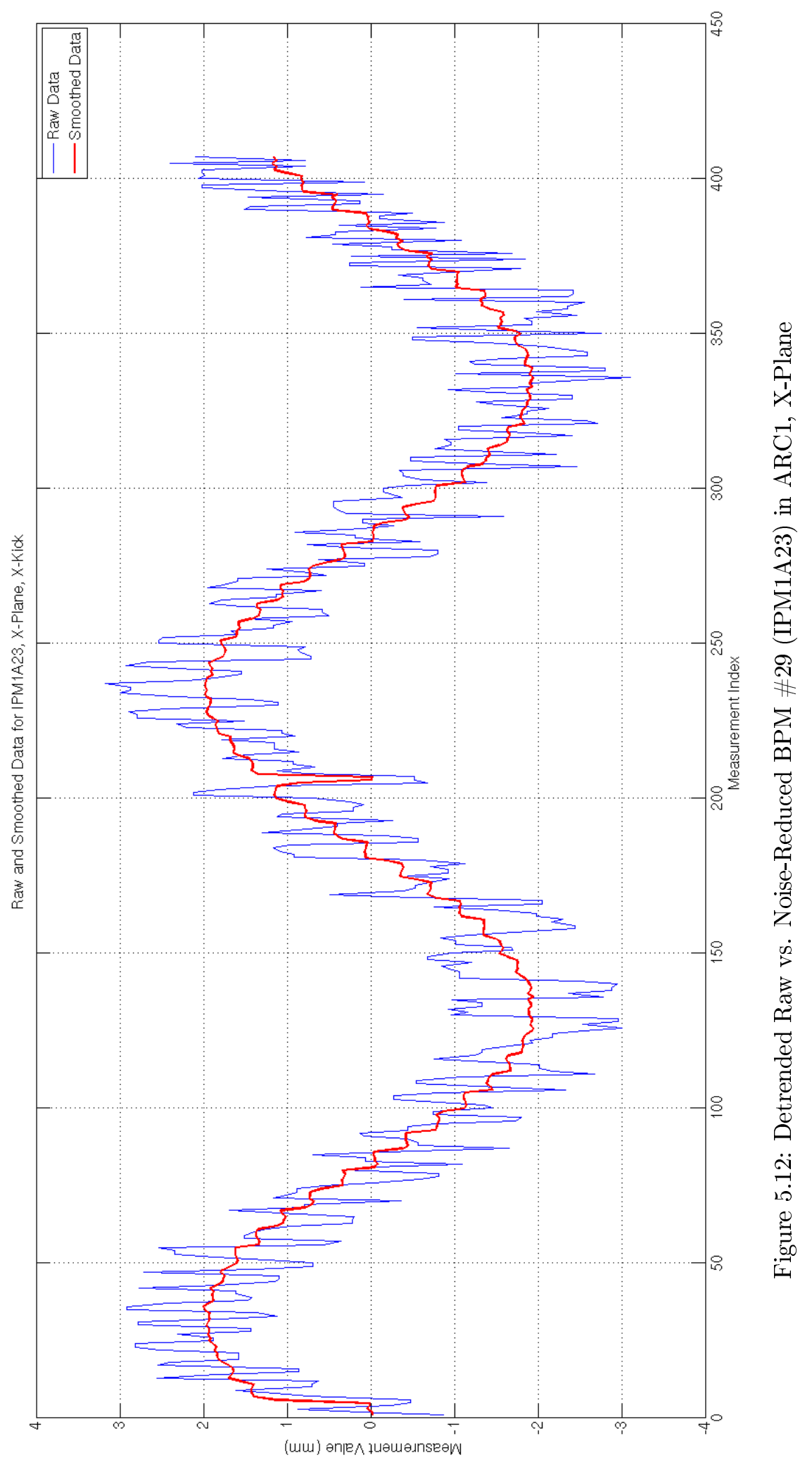




\section{Part III}

\section{Resolution Test}




\section{Chapter 6}

\section{Resolution Test}

In order to demonstrate the utility and power of this technique, as well as test its accuracy, I performed a resolution test. The goal of this test was to both localize and resolve a known change in the optics in the machine. Additionally, this test acts as an example of tuning the machine using the rayTrace procedure. One of the most simple ways to create a known change in the linear optics is to vary a single quadrupole by an amount that is large enough to be detected through system noise, but small enough not to destroy integrity of the beam.

\subsection{Background of Resolution Test}

As described in Section 2.1.2, changing the strength of a single quadrupole will significantly alter the optics of a particle beam. In an open-ended machine, this will alter the downstream behavior of the beam. This change in behavior can be quantified by calculating the Twiss parameters of the beam through the region of the change and comparing them before and after the change is made, as well as to the model optics.

Recalling that the trajectory of a beam in one plane can be described by

$$
\left[\begin{array}{c}
u(z) \\
u^{\prime}(z)
\end{array}\right]=\left[\begin{array}{ll}
C_{u}(z) & S_{u}(z) \\
C_{u}^{\prime}(z) & S_{u}^{\prime}(z)
\end{array}\right]\left[\begin{array}{c}
u_{0} \\
u_{0}^{\prime}
\end{array}\right]
$$

where $C$ and $S$ are cosine and sine functions as previously described, and $u$ is a generalized coordinate. In the thin-lens approximation (focal length, $f$, is much larger than length of magnet, $l$ ), matrix element $M_{21}$ can generally be regarded as the one that describes the strength of the focusing in the transformation. In a pure drift, this term would equal zero, while in a quadrupole it is proportional to the inverse of the focal length, $-\frac{1}{f}$, where $\frac{1}{f}$ is positive for a focusing quadrupole and negative for a defocusing quadrupole. 
In terms of the Twiss parameters, this equation can be described by

$\left[\begin{array}{c}u(z) \\ u^{\prime}(z)\end{array}\right]=\left[\begin{array}{cc}\sqrt{\frac{\beta}{\beta_{0}}}\left(\cos (\psi)+\alpha_{0} \sin (\psi)\right) & \sqrt{\beta \beta_{0}} \sin (\psi) \\ \frac{\alpha_{0}-\alpha}{\sqrt{\beta \beta_{0}}} \cos (\psi)-\frac{1+\alpha \alpha_{0}}{\sqrt{\beta \beta_{0}}} \sin (\psi) & \sqrt{\frac{\beta_{0}}{\beta}}(\cos (\psi)-\alpha \sin (\psi))\end{array}\right]\left[\begin{array}{c}u_{0} \\ u_{0}^{\prime}\end{array}\right]$.

This shows the relationship between the Twiss parameters and the focusing system (recalling that $\gamma \equiv \frac{1+\alpha^{2}}{\beta}$ ). If the focusing strength of a quadrupole is varied, the matrix above will reflect this change. Alternatively, a variation of $\beta$ measured downstream from a quadrupole change can be used to resolve the magnitude of the change in focal strength.

The rayTrace procedure can readily identify local optics changes; this resolution test serves to demonstrate this ability. It also will test the procedure's ability to resolve the magnitude of the change in quadrupole focusing strength. By fitting transfer matrices to the region of the change both before and after the changes, and comparing the resultant matrices to the expected value, the level of accuracy for this procedure can be determined.

\subsection{Experimental Description and Setup}

For this test, I not only wanted to detect an optics change in the machine; I wanted to localize the change and resolve its magnitude. After tune-mode beam was established (10 $\mu \mathrm{A}, 2$ Pass beam), a launchpoint at the beginning of ARC1 was selected (IPM1S05), and two upstream correctors were automatically selected by the rayTrace program. An emittance of $8.00 \times 10^{-6} \mathrm{~cm}$-rad was desired. For the X-plane, this corresponds to an orbit size of $1.314 \mathrm{~mm}$ at $1 \mathrm{~S} 05$. It was decided that 32 points along the launchpoint phase ellipse would allow for adequate resolution, and two turns around the ellipse were taken (for a total of 64 points per plane) to be sure that the beam did not drift during the test and that hysteretic concerns were properly accounted for. All energy and orbit locks were disabled in the relevant areas.

While this process took place, I also ran a Perl script that interacts with the elegant-based model decks to provide a Twiss table of the model optics that can be used to calculate the angular components of the data.

After setting up the machine, a baseline test was run where no aspect of the machine was changed. Once data for both planes was acquired, the setting of a quadrupole toward the end of ARC1 (MQB1R01) was changed $+20 \%$ (from 2994.540 G-cm to 3593.448 G-cm), and rayTrace was run again. After this, the same quadrupole was changed to $-20 \%$ of the original value (down to 2395.632 G-cm), and finally it was set back to the original setting so that a second baseline reading could be acquired. All of these tests ran properly, although there was beam loss during the positive quadrupole change test. The North Recombiner region temporarily lost beam, although it was unrelated to the optics change. There were also several RF trips during the test, but these are a normal occurrence during running and data acquisition is automatically paused 
until beam is restored.

\subsection{Analysis of Resolution Test Data}

\subsubsection{Initial Analysis}

The initial analysis of the data from this test was performed without any noisereduction techniques. Raw data was analyzed directly using the techniques that had been used in previous tests. The data and model information was gathered as described in Section 6.2. This data is automatically formatted in such a manner that each column represents a single BPM, and each row is a measurement. An example of a plot of the raw data for each BPM in Arc 1 (X-Plane) can be seen in Figure 5.3.

This data and model information is then fed into the fitphase program, as described in Section 4.2.3. From this, phase ellipse plots and data on the Twiss parameters are produced, and qualitative checks are performed to make sure that the data obtained from the test is adequate.

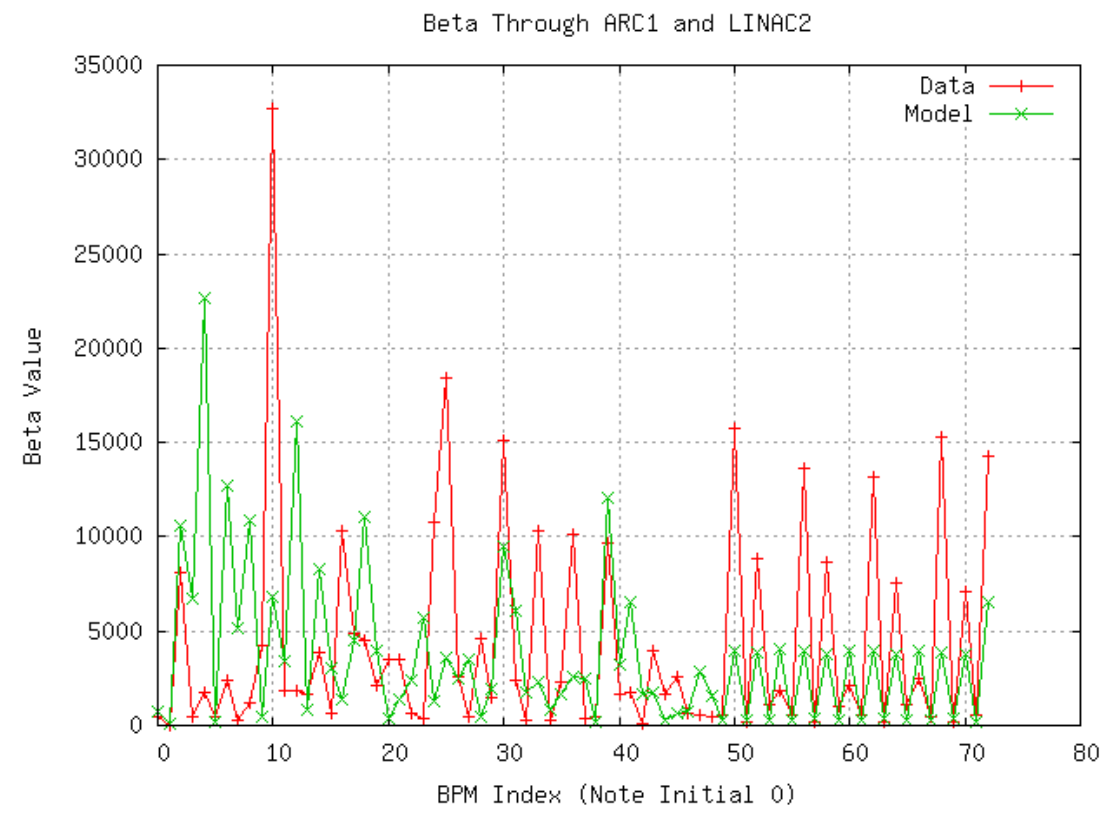

Figure 6.1: $\beta$ Through ARC1 and LINAC2: Comparing Data and Model 


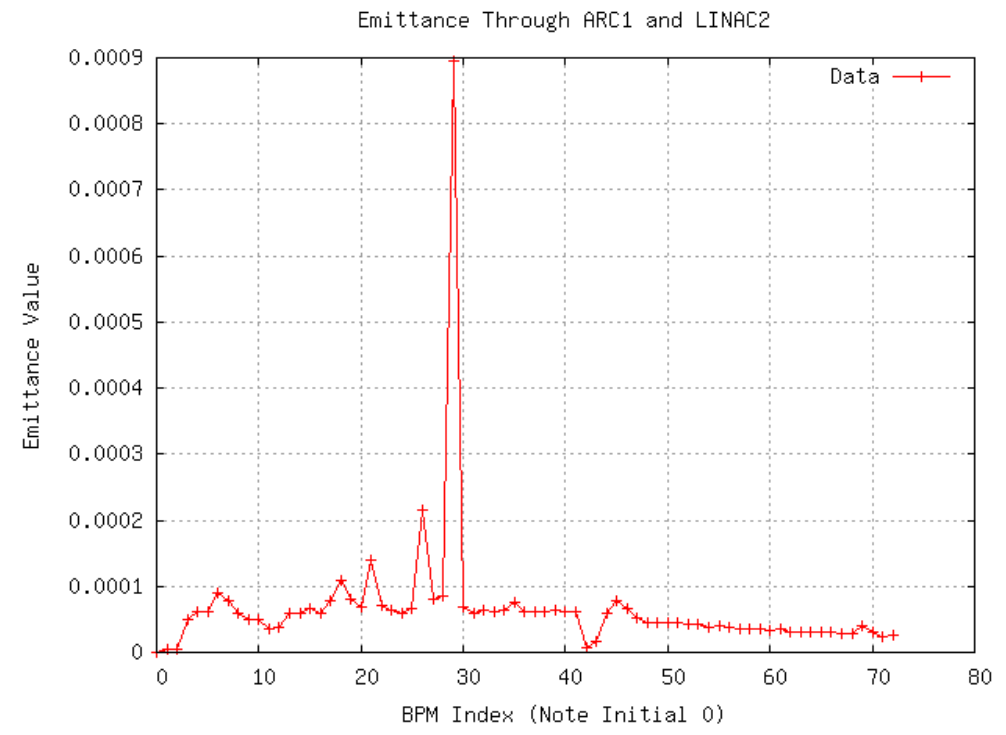

Figure 6.2: Emittance $(\epsilon)$ Through ARC1 and LINAC2: Full Scale

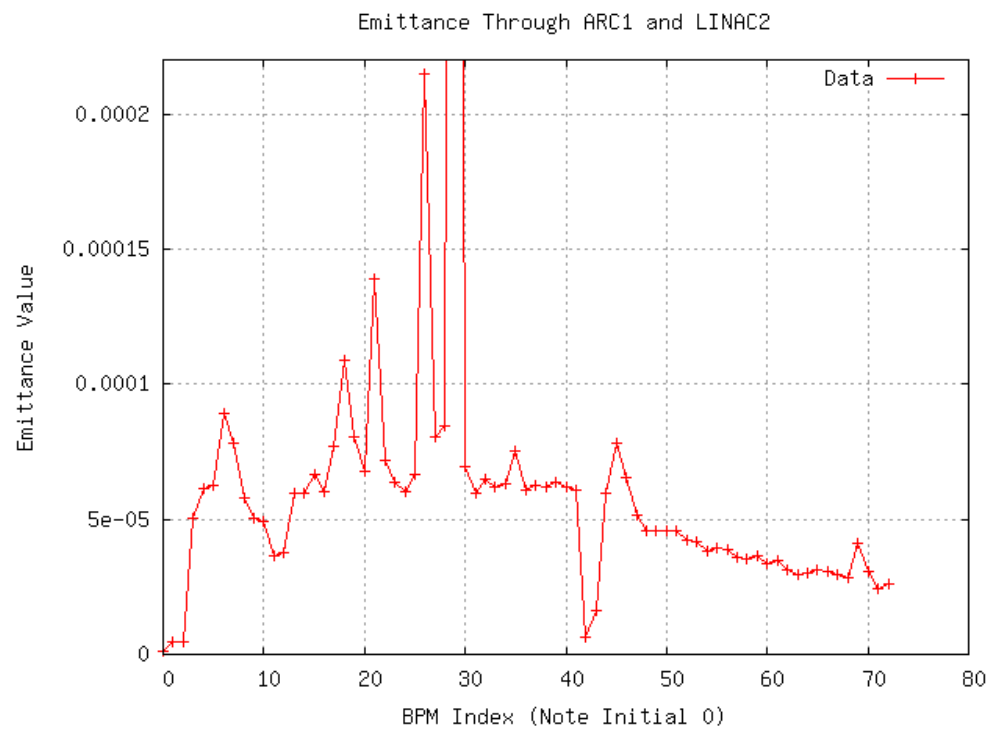

Figure 6.3: Emittance $(\epsilon)$ Through ARC1 and LINAC2: Zoomed In

Looking at the initial data, one can see that the overall trend of $\beta$ (Figure 6.1) and behavior of the emittance (Figures 6.2 and 6.3) acts properly, albeit with higher levels of noise. The $\beta$ peaks in the data generally align with the 
peaks in the model, especially in the linac, where the level of noise is less. The emittance appears to remain relatively constant in the arc, where there is no acceleration, with the exception of some excessively noisy BPMs (indices 28 and 29). Once the emittance enters the linac, it decreases as expected due to acceleration damping. This is encouraging, in that even through the noise of the system, the measurements indicate an overall behavior which is expected.

In looking at this data, it is clear that, most noticeably in the arc, system noise degrades the accuracy of our Twiss parameter measurement. As an example of the phase ellipse created for a single BPM, Figure 6.4 shows that this noise is present both in the measurement of the position and the calculation of the angle found from the position data and the model optics. This dilutes the accuracy of the phase ellipse that is fit to this data, and therefore the calculation of the Twiss parameters.

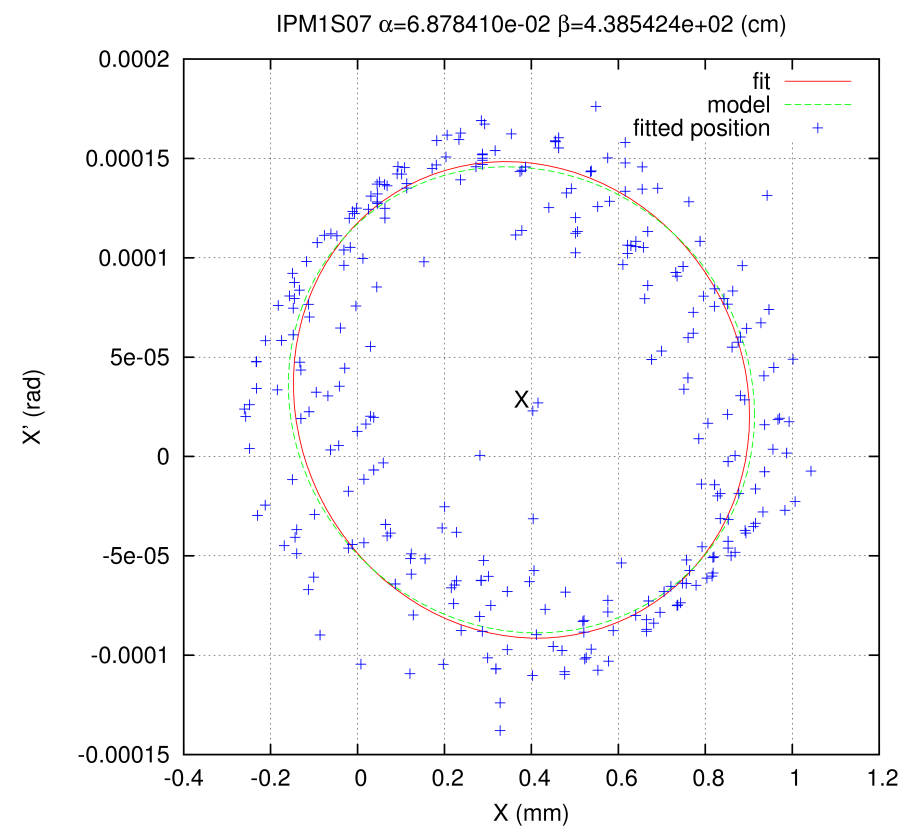

Figure 6.4: Phase Ellipse From Raw Data

In order to improve the accuracy of the Twiss parameter calculations, the noise and errors in the raw data must be reduced. I performed this reduction using singular value decomposition (SVD). 


\subsubsection{Noise-reduced Analysis}

\subsubsection{Comparing Raw and Noise-Reduced Data}

Once the initial analysis was performed, I then detrended the data and reduced the noise, as described in Section 5.2. An example of the detrended, raw data can be seen in Figure 5.4, and the noise-reduced BPM data can be seen in Figure 5.11 .

Comparing first the values of $\beta$ for the raw data and the noise-reduced data (see Figure 6.5), it can be seen that in areas with increased noise such as the center region of ARC1, the SVD analysis has altered the values of $\beta$, while in areas with less noise such as LINAC2, the values are much closer to the original values.

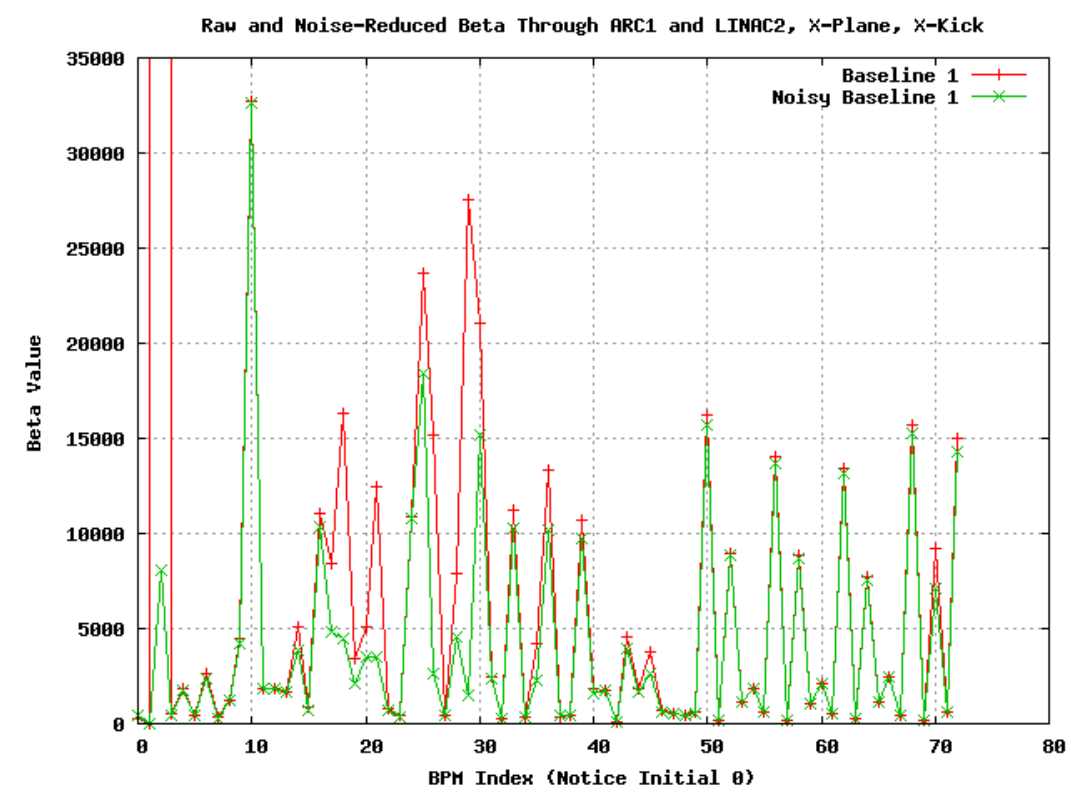

Figure 6.5: Comparison of Raw and Noise-Reduced $\beta$ From Baseline Data

A more-telling manner in which to see the improvement of the Twiss parameter measurements is to compare the emittance as calculated from the raw and noise-reduced data. Figures 6.6 and 6.7 show the level of improvement of the baseline data. Notice that extremely large spikes in the arc have been reduced greatly, and that the overall trend of the emittance can more readily be observed as constant in this region. Furthermore, the proper acceleration damping in the linac is maintained. 


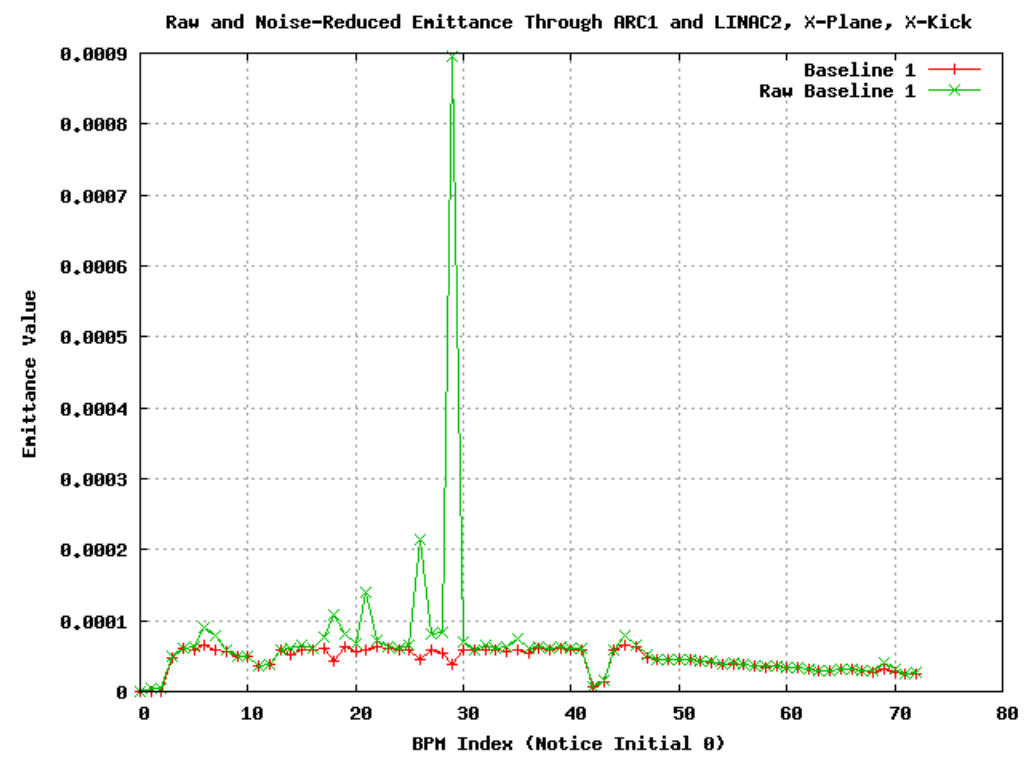

Figure 6.6: Comparison of Raw and Noise-Reduced $\epsilon$ From Baseline Data: Full Scale

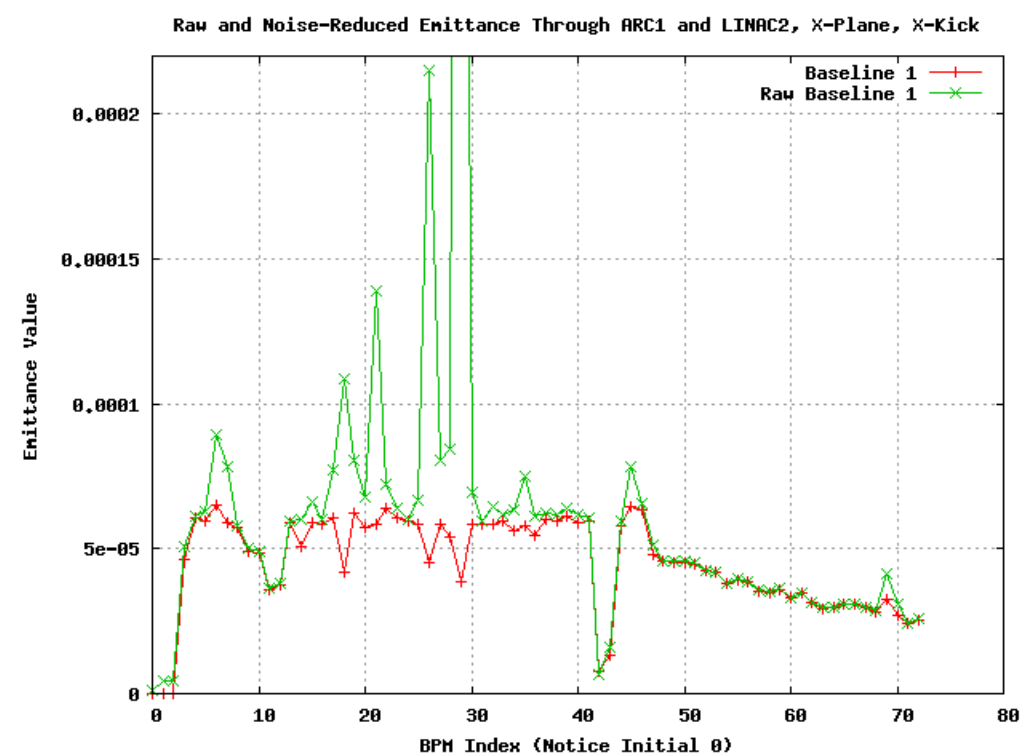

Figure 6.7: Comparison of Raw and Noise-Reduced $\epsilon$ From Baseline Data: Zoomed In 
It is important to note that there are two points in the arc that have low levels of noise where the emittance seems to drop significantly below the rest of the values. The first of these points, located near index 10, can be attributed to the use of switched electrode electronics (SEE) BPMs rather than 4-channel BPMs located throughout the rest of the arc. These BPMs report differently than the 4-channel BPMs, and thus the values will be different. For more on the differences of these BPMs, please see Section 1.3. The second of these dips, located after index 40, is due to the location of the BPMs in the recombiner chicane. At this point, the beamline undergoes a rapid change in direction, and it is believed that these BPMs may be reporting incorrect values due to misalignments.

One further comparison should show the degree to which these signals are cleaned up through this procedure. In Figure 6.8, one can see the before and after images of the same phase ellipse plot for a BPM near the beginning of ARC1. Notice that the center has been moved to the zero point, and that the scale has changed between the two plots. Listed at the top of each plot is the BPM name (IPM1S07), the value for $\alpha$, and the value for $\beta$. In this specific case, the value of $\beta$ has remained nearly the same, but the value for $\alpha$ has changed significantly from 0.069 to 0.203 after the reduction process. Because emittance is proportional to the area of the phase ellipse $(A=\pi \epsilon)$, and emittance can be defined in terms of the Twiss parameters as

$$
\epsilon=\gamma x^{2}+2 \alpha x x^{\prime}+\beta x^{\prime 2},
$$

it is clear that a significant change in $\alpha$ will change the value of the emittance, which is why in the plots comparing the raw and reduced emittances (Figures 6.6 and 6.7 ), the emittance values improved. I used this improved data set to analyze the resolution test.
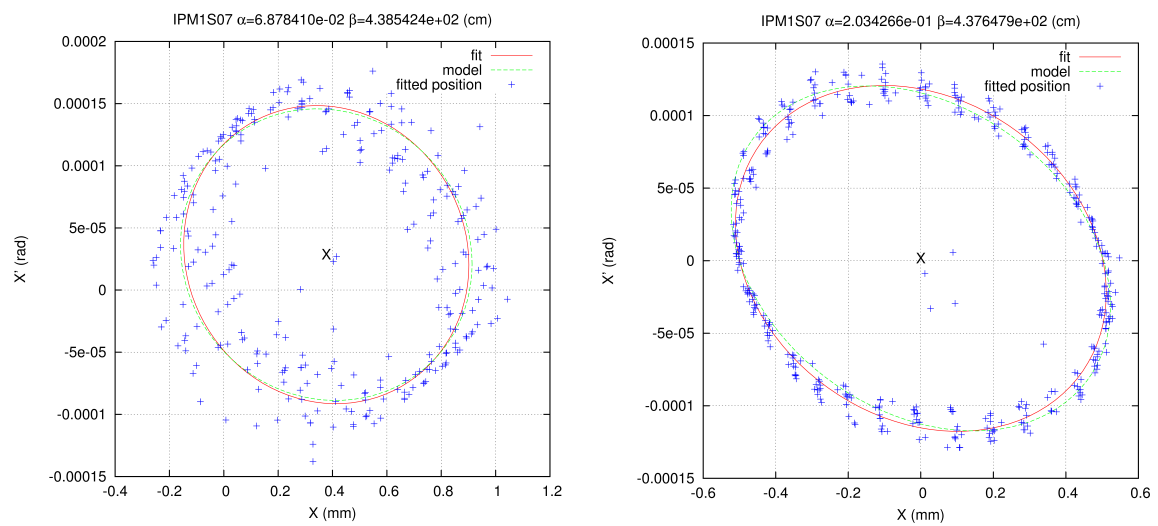

Figure 6.8: Comparison of BPM Phase Ellipse Before and After Noise Reduction 


\subsubsection{Localizing A Known Optics Change}

In order to meet the goals of this experiment, one must be sure that each of the four runs acquired usable data. One way to check for this is to compare the two baseline measurements that were taken prior to and after varying the quadrupole settings, called Baseline 1 and Baseline 2, respectively. Figure 6.9 shows the comparison of the derived emittance, $\epsilon$, for the two baseline tests. From this, it is apparent the the general trends are the same, and that the emittances for each agree well in areas of low noise. It is important to note that the arc region contained several points of higher-noise BPMs (index 23, 28, and 29), which will influence the calculation of the Twiss parameters nearby. Therefore, with several noisy BPMs in the arc, there will be more noise present in the overall region than simply at those BPMs.

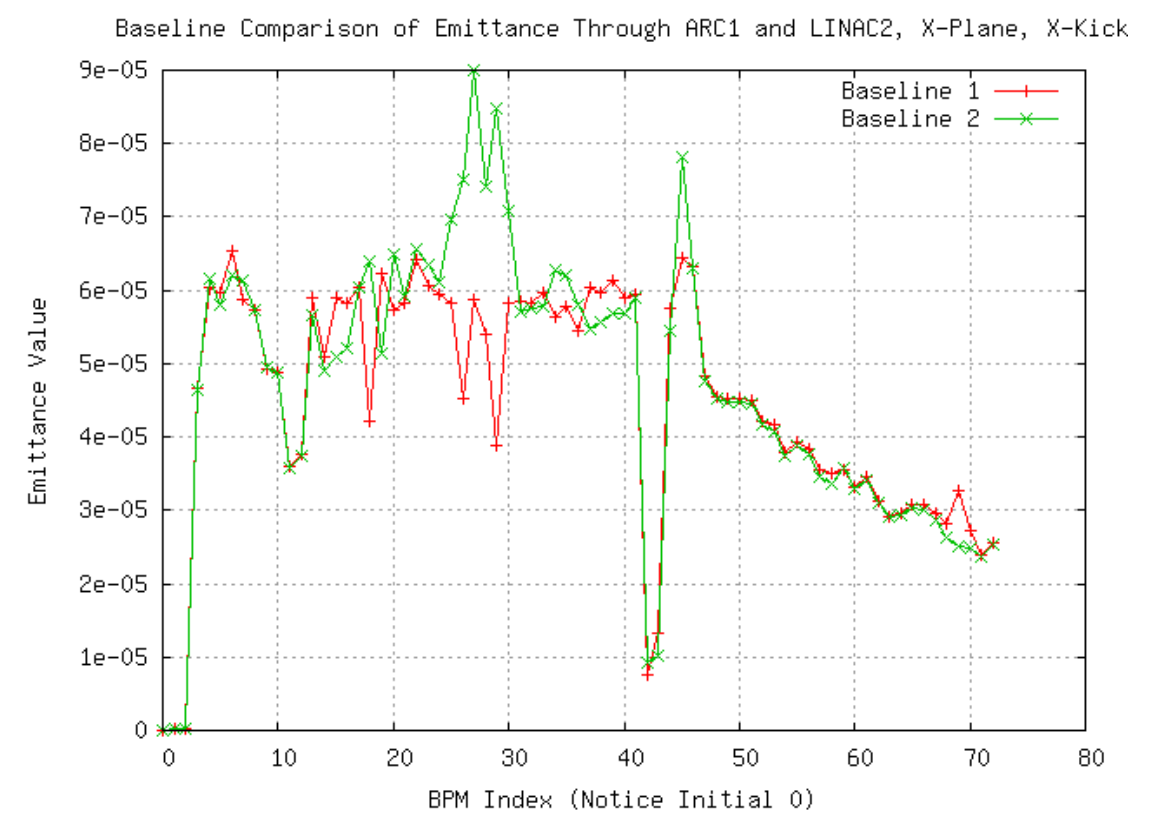

Figure 6.9: Comparison of Baseline $\epsilon$

Looking at a comparison of the baseline $\beta$, values, it can be seen that there is almost a perfect match throughout the arc and the linac. The exception to this match is, again, found in the areas of higher noise. Figure 6.10 shows the plot of the baseline values of $\beta$ through ARC1 and LINAC2. The fact that these two signals overlay through this section of the machine means that this procedure is very reproducible in its results. The two baseline tests took place with two quadrupole changes in between, separated by approximately 45 minutes. 


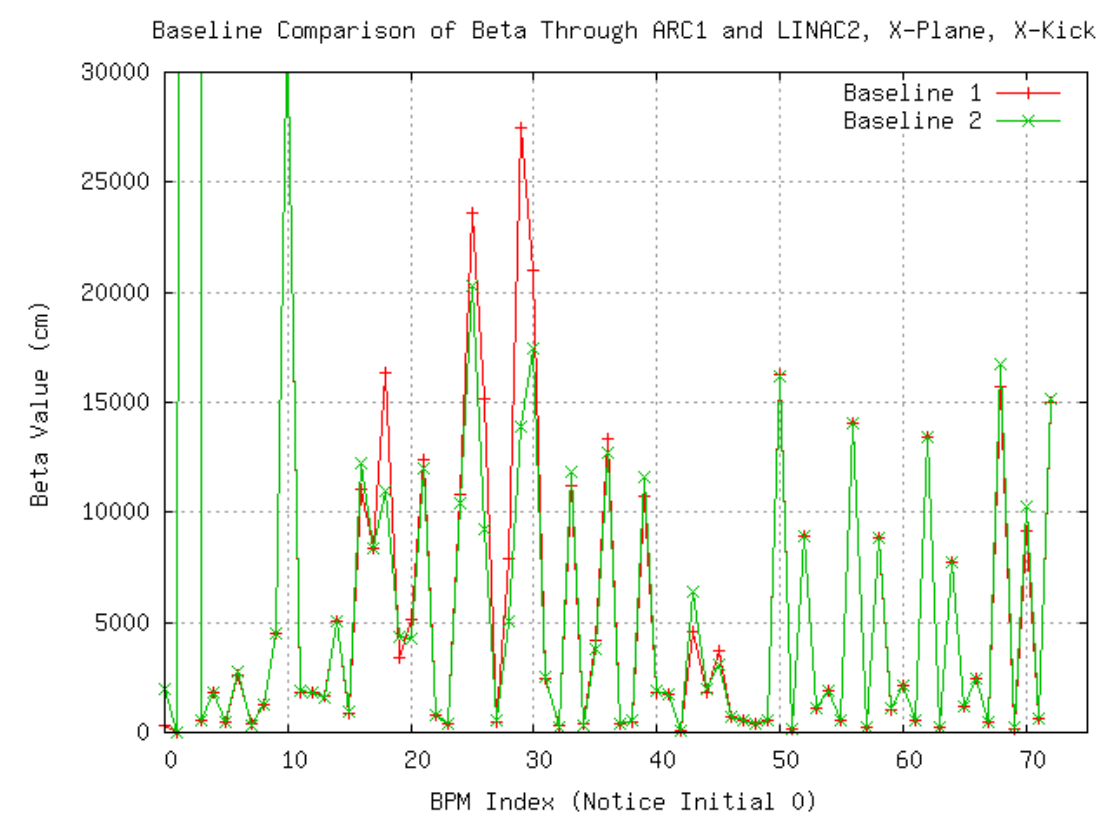

Figure 6.10: Comparison of Baseline $\beta$

Meeting the first goal of the resolution test, the localization of an optics change, is accomplished by overlaying the plots of the calculated $\beta$ values for all four tests. One can clearly see in Figure 6.11 that the values of $\beta$ for all four tests overlay through ARC1, matching nearly perfectly with the exception of the higher-noise regions. Looking at Figure 6.12, it is clear that the overall trend of the emittance remains nearly the same for all four tests, which is expected.

Looking at Figure 6.11, or for a more detailed plot, Figure 6.13, one can see that at index 39, all four points overlay. At index 40, however, two points overlay (the baseline measurements), and the other two points are distinctly separated. This separation maintains itself through the rest of the linac. This means that something in the optics has changed between indices 39 and 40. This is also where the quadrupole that we varied, MQB1R01, is located. Furthermore, the values of $\beta$ for the positive and negative quadrupole changes vary as expected with respect to the baseline values, with the positive change on one side of the baseline and the negative change on the other side. This demonstrates the procedure's ability to localize an optical change, meeting one of the goals of this test. 


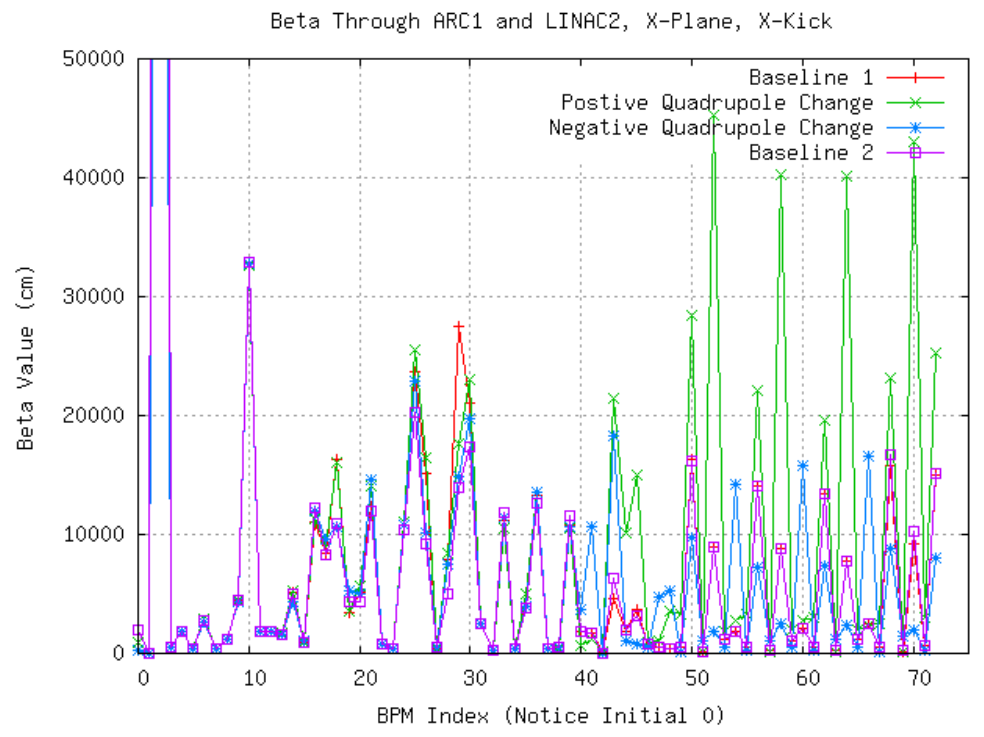

Figure 6.11: Overlaid $\beta$ Through ARC1 and LINAC2

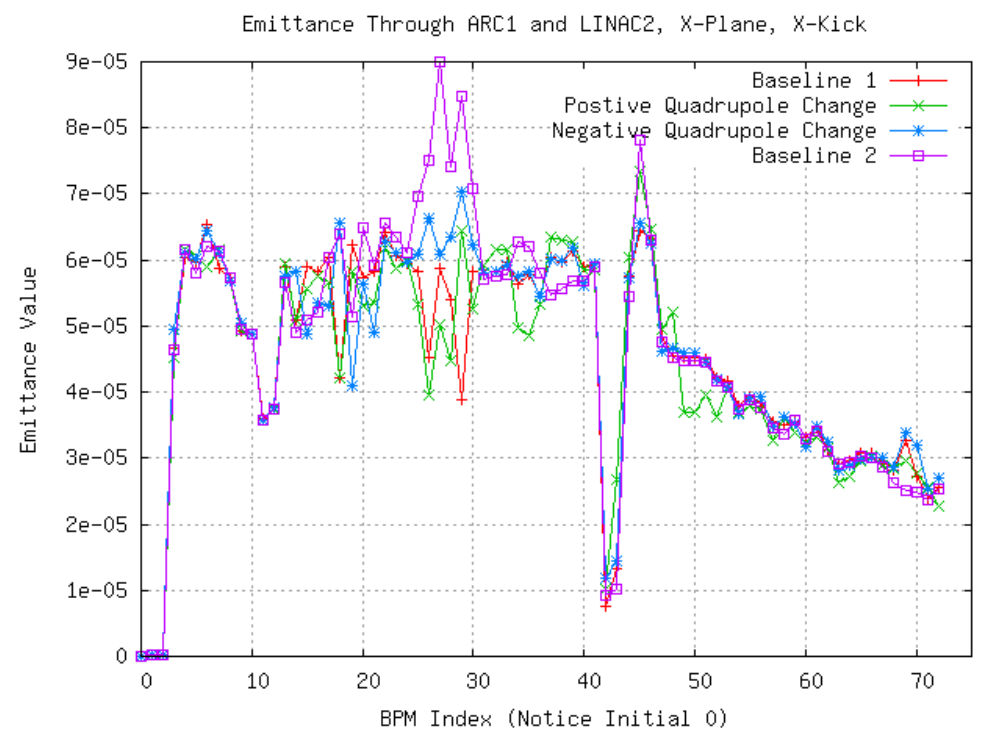

Figure 6.12: Overlaid $\epsilon$ Through ARC1 and LINAC2 


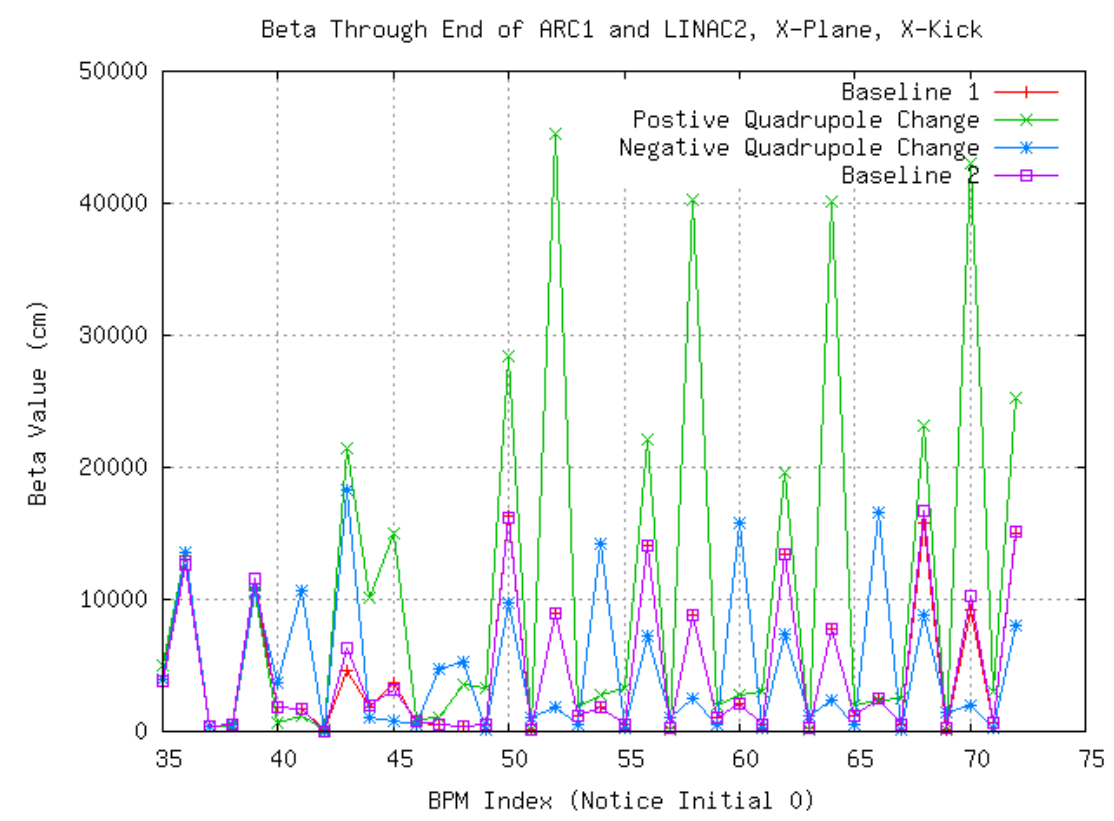

Figure 6.13: Overlaid $\beta$ At End of ARC1 Through LINAC2

\subsubsection{Resolving the Quadrupole Change}

The second goal of this resolution test is to investigate this procedure's ability to find the magnitude of a known change in a quadrupole. The procedure has been successful in finding the location of the optics change that was the result of varying a quadrupole by a known amount. Looking at Figure 6.13, it can be seen qualitatively that the focusing has been changed at this location. This second goal aims to quantify the magnitude of this change.

\subsubsection{Mathematical Review}

In order to describe how the value of the quadrupole strength was determined, a brief mathematical background will be revisited. For simplicity of description, the following mathematical explanation will use the thin lens approximation. However, the fitting routine used to do the real analysis did not use this approximation, and took into account the dimensions and strengths of every element in the relevant area.

In order to find the strength of a given quadrupole from the data gathered and calculated by the rayTrace procedure, knowledge of the local machine and model optics must also be known. To reduce the amount of unknown contributions to the signal, using as short a section of beamline as possible is best, as there are less components that can contribute to the optics. Already having position data at each of the two BPMs surrounding the quadrupole, as well as the 
angular information as calculated by the procedure, calculating the quadrupole strength can be accomplished by extracting it from the relevant transfer matrix component through a least squares fit.

Recalling that the matrix for a driftspace can be written as

$$
M_{d}=\left[\begin{array}{llll}
1 & L & 0 & 0 \\
0 & 1 & 0 & 0 \\
0 & 0 & 1 & L \\
0 & 0 & 0 & 1
\end{array}\right]
$$

and that a thin lens quadrupole can be written as

$$
M_{q}=\left[\begin{array}{cccc}
1 & 0 & 0 & 0 \\
-\frac{1}{F_{x}} & 1 & 0 & 0 \\
0 & 0 & 1 & 0 \\
0 & 0 & -\frac{1}{F_{y}} & 1
\end{array}\right],
$$

where $L$ is the length of the driftspace, and $F_{x}$ and $F_{y}=-F_{x} \equiv-F$ are the focusing terms for the $X$ and $Y$ planes of the quadrupole, respectively, one can multiply these matrices together to find the total transfer matrix of the quadrupole-drift system:

$$
\begin{aligned}
M_{d} M_{q}= & {\left[\begin{array}{llll}
1 & L & 0 & 0 \\
0 & 1 & 0 & 0 \\
0 & 0 & 1 & L \\
0 & 0 & 0 & 1
\end{array}\right]\left[\begin{array}{cccc}
1 & 0 & 0 & 0 \\
-\frac{1}{F} & 1 & 0 & 0 \\
0 & 0 & 1 & 0 \\
0 & 0 & \frac{1}{F} & 1
\end{array}\right], } \\
= & {\left[\begin{array}{cccc}
1-\frac{L}{F} & L & 0 & 0 \\
-\frac{1}{F} & 1 & 0 & 0 \\
0 & 0 & 1+\frac{L}{F} & L \\
0 & 0 & \frac{1}{F} & 1
\end{array}\right]=M . }
\end{aligned}
$$

This transfer matrix can then be used to find the trajectory of the particle beam as

$$
\left[\begin{array}{l}
x_{2} \\
x_{2}^{\prime} \\
y_{2} \\
y_{2}^{\prime}
\end{array}\right]=\left[\begin{array}{cccc}
1-\frac{L}{F} & L & 0 & 0 \\
-\frac{1}{F} & 1 & 0 & 0 \\
0 & 0 & 1+\frac{L}{F} & L \\
0 & 0 & \frac{1}{F} & 1
\end{array}\right]\left[\begin{array}{l}
x_{1} \\
x_{1}^{\prime} \\
y_{1} \\
y_{1}^{\prime}
\end{array}\right]
$$

Instead of using this equation to find the trajectories at the second (downstream) point, one can use knowledge of the trajectories at both the upstream and downstream points to find the terms of the transfer matrix. If there is knowledge of some of the terms of the transfer matrix, such as the length of the drift, then one can find the other terms. This is how the focusing term was found.

For the quadrupole that was changed, MQB1R01, the trajectory information for the upstream (IPM1R01) and downstream (IPM1R02) BPMs was used in combination with knowledge of the drift length (and quadrupole length) to calculate the focusing term of the transfer matrix. After reducing the noise of 
the BPM data using SVD (see Chapter 5 and Section 6.3.2), the angular information was calculated with fitphase, as described in Section 4.2.3. Because this angular information depends upon the accuracy of the model, it was decided that it was only necessary to use this information at the upstream BPM. This is possible because the linear optics at CEBAF are uncoupled, and only one angular term per plane is necessary to find the focusing term. This can be seen in looking at the equation for the $X$ plane:

$$
\begin{aligned}
M_{11} x_{1}+M_{12} x_{1}^{\prime}+M_{13} y_{1}+M_{14} y_{1}^{\prime} & =x_{2}, \\
\left(1-\frac{L}{F_{x}}\right) x_{1}+L x_{1}^{\prime}+0+0 & =x_{2}, \\
\left(1-\frac{L}{F_{x}}\right) x_{1}+L x_{1}^{\prime} & =x_{2} .
\end{aligned}
$$

Similarly for the $Y$ plane:

$$
\left(1-\frac{L}{F_{y}}\right) y_{1}+L y_{1}^{\prime}=y_{2} .
$$

One can then solve Equations 6.7 and 6.8 for the focusing terms.

To do this for all of the data points at both BPMs, and to take into account the thickness of the lens and other components of the beamline between IPM1R01 and IPM1R02, a Mad-X fitting routine was written that using the same principles just described.

\subsubsection{Fitting With Mad-X}

Equations 6.7 and 6.8 can be rewritten by putting all of the terms on one side of the equation. In general coordinates, this looks like

$$
u_{2}-M_{i i} u_{1}-M_{k l} u_{1}^{\prime}=0
$$

where $u$ is either $x$ or $y, M_{k l}$ is $M_{12}$ for the $x$ plane or $M_{34}$ for the $y$ plane, and $M_{i i}$ is $M_{11}$ for the $x$ plane and $M_{33}$ for the $y$ plane. Since the values of $u_{1}$ and $u_{2}$ are known from the BPM readings (and the noise has been reduced using SVD), $u_{1}^{\prime}$ has been calculated from the smoothed data by fitphase, and $L$ is known from the model, the only unknown term is $M_{i i}$, which contains the quadrupole focusing term. For focusing in the $X$-plane, one defines $k=\frac{1}{B \rho} \frac{\partial B_{y}}{\partial x}$

and recalls that in a quadrupole, $\frac{\partial B_{x}}{\partial y}=-\frac{\partial B_{y}}{\partial x}$. Then $M_{i i}$ for a lens of length $l$ may be written as

$$
\begin{aligned}
& M_{11}=\cos (\sqrt{k} l)-\sqrt{k} L \sin (\sqrt{k} l) \\
& M_{33}=\cosh (\sqrt{k} l)+\sqrt{k} L \sinh (\sqrt{k} l) .
\end{aligned}
$$

Here $B \rho$ is the magnetic rigidity of a central reference orbit and depends upon the momentum of the particle beam and is constant when the momentum is constant in the magnet. Thus, the only unknown term in Equation 6.9 is the quadrupole strength. 
To find this quadrupole strength, a fitting routine was written in Mad-X which varied the strength of the quadrupole in order to satisfy

$$
\sum_{m=1}^{N}\left(u_{2}^{m}-M_{i i} u_{1}^{m}-M_{k l} u_{1}^{\prime m}\right)^{2}=0
$$

for each plane, where $m$ is the total number of data points. Since $u_{2}$ is a measured value, and $M_{i i} u_{1}+M_{k l} u_{1}^{\prime}$ is a value that is calculated by the fitting routine and must equal $u_{2}$, then satisfying Equation 6.12 is analogous to minimizing a $\chi^{2}$. It is important to note, however, that because the values used for $u$ in this process have already been smoothed using singular value decomposition, this is not a statistical $\chi^{2}$ minimization. Instead, the $\chi^{2}$ in this instance represents a function to be minimized. Furthermore, recalling that

$$
\chi^{2}=\sum \frac{1}{\sigma^{2}} \frac{\left(x_{f}-x_{c a l c}\right)^{2}}{(N-1)},
$$

one can solve for $\sigma$, which would give an estimate (in units of distance from beam pipe center) of the error for the system of BPMs used in the calculation:

$$
\sigma=\sqrt{\chi^{2} \sum \frac{\left(x_{f}-x_{c a l c}\right)^{2}}{N-1}} .
$$

In the Mad-X script, which is shown in Appendix B.1.2, the input files include $x, x^{\prime}, y$, and $y^{\prime}$ data for both of the BPMs used for the fitting; in this case IPM1R01 and IPM1R02. Although included in the input file, the angular information is not used for the second BPM. A lattice file, complete with beamline components, their lengths, and any effects they may have on the optics, is also an input. The script uses this lattice file to define the parameters of the beamline between the two BPMs, including the quadrupole that was changed for this test, MQB1R01. The momentum, which is constant through the Arc sections of the accelerator, is defined for this section of beamline as $p c=0.61$ $\mathrm{GeV}$, which then defines $B \rho$, as $B \rho=\frac{p c}{0.2998}[6]$. Mad-X then calculates the Twiss parameters and transfer matrix between the BPMs.

The script then loops through each data point and calculates $\left(u_{2}-M_{i i} u_{1}-\right.$ $\left.M_{k l} u_{1}^{\prime}\right)^{2}$ for each plane, as well as for both planes together. Each iteration is then added together, giving $\sum\left(u_{2}-M_{i i} u_{1}-M_{k l} u_{1}^{\prime}\right)^{2}$. Looking at Equation 6.9, one can see that this sum must equal zero. The Mad-X routine will minimize this function, reaching a value close to zero:

$$
\sum_{m=1}^{N}\left(u_{2}^{m}-M_{i i} u_{1}^{m}-M_{k l} u_{1}^{\prime m}\right)^{2}=\sum_{m=1}^{N} \Delta_{m}^{2} .
$$

Equation 6.12 is set as a constraint in the fitting routine. All other terms are constants. The script will then vary only the strength of the MQB1R01 quadrupole in order to meet the conditions defined by the lattice and Equation 6.15. Once the conditions are met, the quadrupole strength is recorded, then 


\begin{tabular}{|c|c|c|c|c|}
\hline MQB1R01 & Baseline 1 & Positive 20\% & Negative 20\% & Baseline 2 \\
\hline Calculated Quad Value (Gauss) & 2937.63 & 3508.57 & 2357.89 & 2915.46 \\
\hline Percent Change From Baseline 1 & 0 & 19.44 & -19.73 & 0.75 \\
\hline Archived Quad Value (Gauss) & 2994.62 & 3593.45 & 2395.63 & 2994.63 \\
\hline Sigma (microns) & 30.69 & 50.83 & 37.81 & 53.17 \\
\hline
\end{tabular}

Table 6.1: Summary of Results

converted to gauss. In order to perform this minimization and find $M_{i i}$, one can take the derivative of Equation 6.12 with respect to $M_{i i}$ :

$$
\begin{gathered}
\sum_{m=1}^{N}\left(u_{2}^{m}-M_{i i} u_{1}^{m}-M_{k l} u_{1}^{\prime m}\right) u_{1}^{m} \equiv 0 \\
-M_{i i} \sum_{m=1}^{N}\left(u_{1}^{m}\right)^{2}=M_{k l} \sum_{m=1}^{N} u_{1}^{\prime m} u_{1}-\sum_{m=1}^{N} u_{1}^{m} u_{2}^{m}, \\
M_{i i}=\frac{1}{\sum_{1}^{N}\left(u_{1}^{m}\right)^{2}}\left[\sum_{m=1}^{N} u_{1}^{m} u_{2}^{m}-M_{k l} \sum_{m=1}^{N} u_{1}^{\prime m} u_{1}^{m}\right] .
\end{gathered}
$$

The final value of Equation 6.15 should be close to zero. Dividing this value by the number of data points and taking the square root should give a value for $\sigma$ :

$$
\sigma=\sqrt{\frac{\sum_{1}^{N} \Delta_{m}^{2}}{N-1}} .
$$

Converted to units of micrometers, this value roughly estimates the resolution error of the BPM system in this section that remains after SVD analysis for each of the four test runs.

In order for this test to be successful, the values of the quadrupole calculated through this fitting routine must show a change in the quadrupole setting that is close to the $20 \%$ change that was entered into the control system. The results of this test are summarized in the table in Table 6.1. The calculated quadrupole value in this table is that calculated for both planes simultaneously. The archived quadrupole value is that reported by the archive system for the time of the test. $\sigma$ is calculated for each of the four test runs. It is clear from looking at this table that the $20 \%$ quadrupole change in both the positive and negative directions is very nearly resolved, with all values being less than $1 \%$ away from the expected value of $\pm 20 \%$ for the Positive and Negative test run and $0 \%$ for the Baseline 2 test run. 


\subsubsection{Uncertainty Analysis}

An accelerator system is a complex arrangement of components that contribute to the noise and uncertainty of any measurement that is taken. In addition to errors and noise in the components themselves, external factors such as vibrations, temperature, and electrical fluctuations will increase the contribution of uncertainty to any measurement. In order to quantify the uncertainty that may remain in the final results of this test, the same procedure was performed on several quadrupoles which, according to the archive, remained at a constant setting throughout the four test runs. These quadrupoles share common traits with MQB1R01; all are in the Arc 1 region of the accelerator, and all are the only quadrupole between the adjacent BPMs. All of these quadrupoles are located upstream of MQB1R01, which means that the BPM readings for all four test runs should be the same.

The results of this uncertainty analysis is shown in Table 6.2. Each row in this table contains the data for each of the five quadrupoles that were held constant for this analysis. The four columns labeled with the different test runs (Baseline 1, Positive, Negative, and Baseline 2) show the value of the quadrupole setting in gauss as calculated by the Mad-X routine from the data. The column labeled "Average" is the average quadrupole strength from the four test runs for each quadrupole. The "Archived Value," also in gauss, is the value that the quadrupole was set at according to the machine archive. The "Avg. \% Diff of Arch." is the percent difference between the average quadrupole setting and the archived quadrupole setting. The "Spread" is the range of values of the quadrupole strength calculated from the four test runs. The "Spread \% of Arch." and "Spread \% of Avg." columns are a measure of the size of the spread compared to the archived and average values of the quadrupoles, respectively. Finally, the "Avg. Sigma" column is the average value of the calculated $\sigma$ for each of the quadrupoles, measured in micrometers.

Looking at the average value of the quadrupole setting compared to the archived value, one can see that the calculated values are near the archived value, with the largest deviation occurring at $1 \mathrm{~A} 38$. One cannot expect to calculate a value that is exactly the same as the value projected from the archive, as there are unknown levels of error in the archived value as well as the calculated value. This comparison is mostly performed to make sure the values that are being calculated are in the right neighborhood.

A more important measure is the spread of the values calculated for the four test runs. A large spread in these values would mean that the reproducibility of the quadrupole value calculation is poor, while a small spread would mean greater reproducibility. One would expect the spread to be only a few percent of the average or archived value, as the stability range of the quadrupole strength is also of this magnitude. For the five quadrupoles that were included in this study, the spread of the quadrupole values ranged from 30-109 gauss, or approximately $1-5 \%$ of the average calculated value. Four out of the five quadrupoles had spreads that were near $1 \%$ of the average value.

The $\sigma$ values estimate the level of uncertainty that remains in the BPM 
system used for each calculation. For each of these calculations, two BPMs were used; one upstream of the quadrupole and one downstream. The BPMs are designed to perform within a $\sigma=50 \mu \mathrm{m}$ specification range. After smoothing the data using SVD, this value is expected to decrease by some unknown amount. One would still expect a $\sigma$ in the tens of micrometers range, as all noise and error contributions are not smoothed out in the SVD procedure. For four of the five BPM pairs, $21 \mu m \leq \sigma \leq 32 \mu m$, which is a reasonable estimation of the remaining uncertainty of the BPM pair system. For the BPM pair surrounding MQB1A29, $\sigma \approx 80 \mu \mathrm{m}$, which is higher than the $50 \mu \mathrm{m}$ specification. This means that one or both of the BPMs, or some other system which influences the BPMs around this quadrupole have a higher-than-expected uncertainty, even after the uncorrelated signal contributions have been removed with SVD.

One possible cause of this extra noise is a very small RMS beam size in one plane at either BPM. Looking at Figure 6.14, one can see that in the X-plane, the RMS beam size for IPM1A29 (at BPM Index 32 in the figure) is smaller than anywhere else in the area. This means that the measurement at IPM1A29 will have a greater noise contribution. In fact, both quadrupoles that use IPM1A29 in the calculation (MQB1A28 and MQB1A29) have higher $\sigma$ calculations, which strengthens the argument that the small beam size in the X-plane at IPM1A29 contributes to the overall uncertainty and a higher value for $\sigma$. The effect of this noise from BPM IPM1A29 will be magnified when looking at the MQB1A29 quadrupole because of its close proximity to the magnet itself. At CEBAF, beam position monitors are generally placed just before the quadrupole, with little to no drift space in between the end of the BPM and the beginning of the quadrupole, such as in Figure 1.4. IPM1A29 is located on the same girder as MQB1A29, and its noise level is high compared to its signal due to the small beam size. A noisy signal entering a quadrupole will lead to a greater number of possible trajectories through the lens, which can in turn reduce the accuracy of the fit.

This is reflected in the value of $\sigma$. Looking back at the definition of $\sigma$, Equation 6.17, one can see that, given the same number of data points, a larger value of $\sum_{1}^{N} \Delta_{m}^{2}$ will result in a larger $\sigma$. This summation will be larger, as the minimization process will yield a larger number because the fit is not as good. If the BPMs in system $a$ have less noise in them than the BPMs in system $b$, system $a$ will have a larger value for $\sum_{1}^{N} \Delta_{m}^{2}$ :

$$
\begin{aligned}
\sigma_{a} & =\sqrt{\frac{\sum_{1}^{N} \Delta_{m, a}^{2}}{N-1},} \\
\sigma_{b} & =\sqrt{\frac{\sum_{1}^{N} \Delta_{m, b}^{2}}{N-1}}, \\
\sum_{1}^{N} \Delta_{m, a}^{2} & >\sum_{1}^{N} \Delta_{m, b}^{2}, \\
\therefore \sigma_{a} & >\sigma_{b} .
\end{aligned}
$$




\begin{tabular}{|c|c|c|c|}
\hline 商 & 命 & 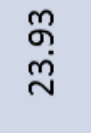 & \\
\hline 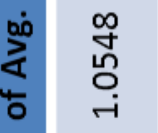 & & 茖 & ఫ \\
\hline 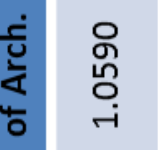 & 总 & 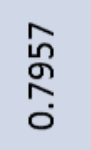 & 范 \\
\hline 离 & 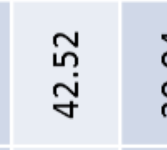 & 营 & 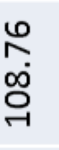 \\
\hline 웅 & i⿱ & $\stackrel{\circ}{\circ}$ & 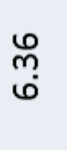 \\
\hline & 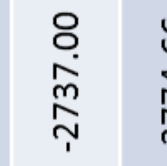 & 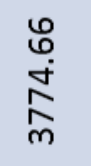 & $\stackrel{\bar{v}}{\Im}$ \\
\hline 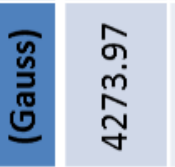 & 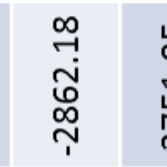 & 量 & 辛 \\
\hline & 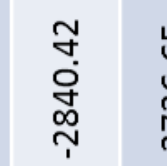 & 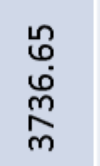 & 兽 \\
\hline 泾 & 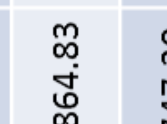 & ְ̊ำ & 望 \\
\hline & 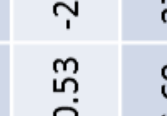 & & 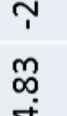 \\
\hline & , & & \\
\hline & & 总 & 8 \\
\hline & & & \\
\hline
\end{tabular}




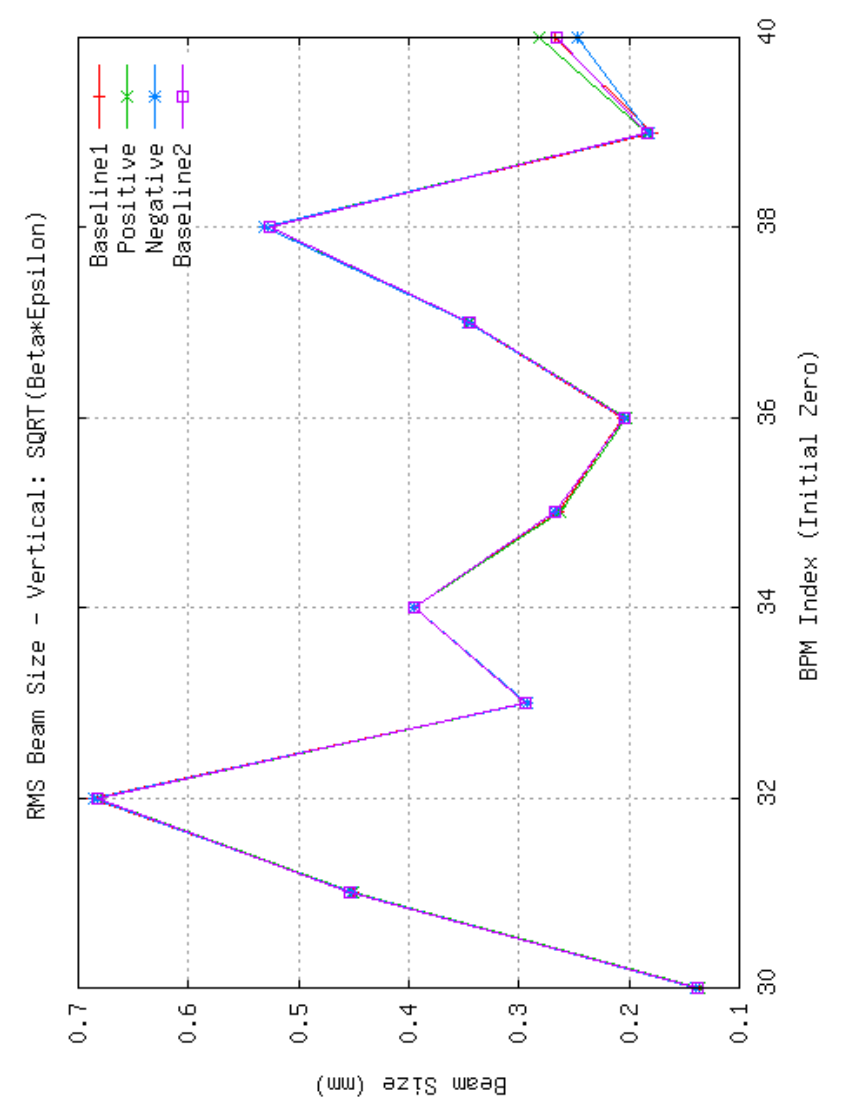

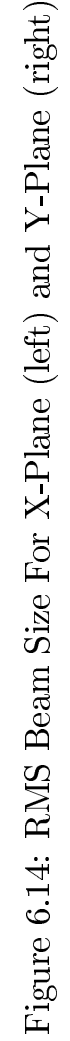

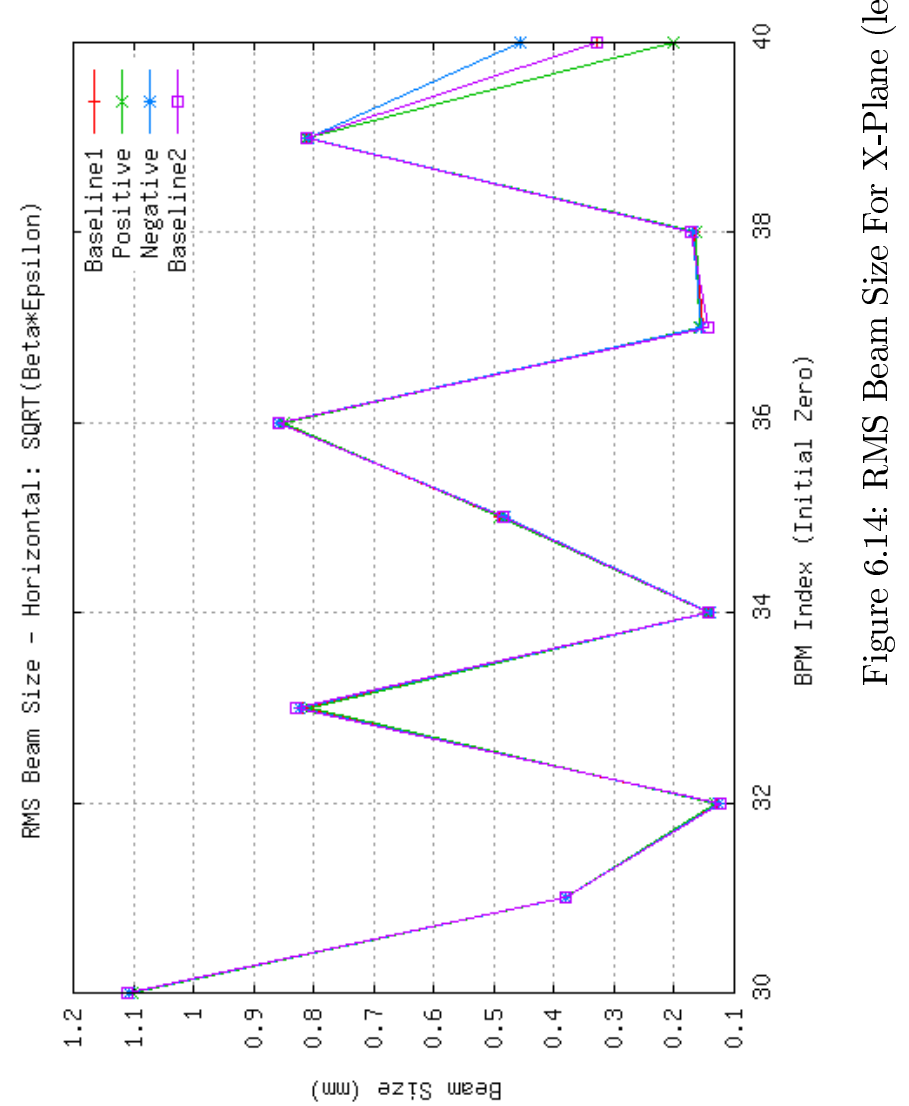




\subsubsection{Interpretations}

There were two main goals for this resolution test. The first is to localize a known optics change. The second is to resolve this known optics change. Both of these goals are important, as the accelerator operations group would need to be able to perform this sort of task with a characterization tool.

The rayTrace procedure was able to clearly localize the optics change to an area between the two beam position monitors, IPM1R01 and IPM1R02. This is where the quadrupole was changed for this experiment. Looking at Figures 6.11, 6.13, and 6.14, it is clear both from the plots of $\beta$ and the plots of the RMS beam size that two Baseline measurements remained overlaid, while the Positive quadrupole change and Negative quadrupole change measurements diverged in opposite directions from the Baseline trajectory after IPM1R01 (at BPM Index 39) and remained separated throughout the South Linac. The Baseline measurements remained along the same trajectory. These trajectories all behave as expected, given the nature of the optics change.

Had the Positive and Negative trajectories not remained separated, or had the Baseline trajectories deviated from each other after this point, one would have to assume other changes had been made in the optics of the machine, or that significant errors were present. One can see the effects of errors between BPM Index 20 and 30 in Figures 6.9, 6.10, 6.11, and 6.12. In the plots of emittance, there are clear differences between the two Baseline measurements. Similarly, in the plots of $\beta$, the values should overlay in this region but do not. This is because of the increased error present in some of the BPMs in this region.

In the region of the quadrupole change however, the BPM noise was comparable to that of the rest of Arc 1. This is fortunate, as it allowed for a straightforward identification of the optics change. Had the change occurred in an area of higher noise, localization would have likely been possible, though perhaps not as precise. For example, the change may have been localized to within a range of a few BPMs instead of between two.

The rayTrace procedure was also able to resolve the magnitudes of the changes set into the strength of quadrupole MQB1R01. Table 6.1 shows that for both the 20\% Positive and 20\% Negative changes in quadrupole strength, the rayTrace procedure could resolve better than a $19 \%$ change in each direction. Furthermore, the two Baseline values are within $1 \%$ of each other, which demonstrates both the high level of reproducibility in MQB1R01 and the resolution that this procedure is able to achieve. Additionally, the values of $\sigma$ range from just over $30 \mu \mathrm{m}$ to just over $53 \mu \mathrm{m}$, and average to $43 \mu \mathrm{m}$, which is a very reasonable level of noise among the BPM pair surrounding the quadrupole.

Looking at Table 6.2, one can see that the spread of the quadrupole values calculated from four different test runs for five different quadrupoles are a small percent of the average quadrupole value. Four of the five quadrupoles had spreads that were less than $1.5 \%$ of the average quadrupole strength. Only one quadrupole had a spread that was larger, and it was under $5 \%$. These small spreads confirm the rayTrace procedure's ability to resolve the strength of a quadrupole, and also demonstrate the stability of the quadrupoles. 
All of the values for $\sigma$ are reasonable, most falling between $\sim 20-30 \mu \mathrm{m}$, and one quite a bit higher at $\sim 80 \mu \mathrm{m}$. Since the specifications for the BPM system have a value of $\sigma=50 \mu \mathrm{m}$ for each BPM, and singular value decomposition is performed on the data prior to fitting, one would expect $\sigma$ for a pair of BPMs to be on the same order as a single BPM. If one or more of the BPMs has a greater noise contribution, either due to a small signal-to-noise ratio or due to noise in the electronics itself, this value will increase, as happened for the BPMs surrounding MQB1A29.

Had the resolution test been performed using MQB1A29 instead of MQB1R01, there would have been an increased uncertainty in the BPM readings, and this would result in a higher value for $\sigma$. This may have reduced the measurement resolution of the quadrupole change.

\subsubsection{Conclusions of Resolution Test}

The resolution test succeeded in meeting its two primary goals. Using the rayTrace procedure, a known optics change was both localized and resolved. The change was determined correctly to be between IPM1R01 and IPM1R02. Since the only component of the beamline between these two beam position monitors that could cause a change in the optics is quadrupole MQB1R01, the observed change in linear optics must be due to this quadrupole.

The strength of MQB1R01 was varied in the control room from a Baseline value by $\pm 20 \%$ and then back to its Baseline value. Data was collected according to the protocols of the rayTrace procedure. The uncorrelated contributions to the BPM signal, including system noise and other unknown contributions, were removed using singular value decomposition. This smoothed data was then used as an input into the fitphase program, where the angular components of the phase ellipse were calculated. Using this $\left(u, u^{\prime}\right)$ data, the Twiss parameters were calculated. Overlaying the plots of $\beta$ for each test run showed that, with the exception of areas with highly noisy BPMs, $\beta$ for all four test runs overlaid throughout Arc 1 until IPM1R01. At this point, the two Baseline test runs remained overlaid, while the Positive test run and Negative test run diverged from the Baseline values in opposite directions and remained separate through the South Linac. This separation occurred at one specific point, and so the optics change must have occurred between IPM1R01 and IPM1R02. This is exactly where the quadrupole was varied.

Next, the $\left(u, u^{\prime}\right)$ data, along with a lattice file containing all of the elements between IPM1R01 and IPM1R02, inclusive, used as inputs to a fitting routine in the Mad-X program. This program used the input data as a set of constraints defining the beamline in this area. Then the fitting routine varied the strength of quadrupole MQB1R01 until the constraints were met as well as possible. Once the constraints were met, the value of the quadrupole was recorded in units of gauss. The approximate noise of the BPM pair surrounding the quadrupole was also recorded. These values were recorded for each of the four test runs. The approximate noise was within a reasonable range for all cases, and the quadrupole values were close to the value indicated by the machine archive. The 
calculated change of the quadrupole value through the four test runs were very close to the expected values. The Positive and Negative quadrupole strength changes were $20 \%$ as indicated by the control room settings and archive values. The calculated values for these were over $19 \%$ for both cases. The second Baseline value was less than $1 \%$ different than the original Baseline value.

Uncertainty analysis was performed using five quadrupoles which remained set at a constant value during the four test runs. All five of these quadrupoles are upstream of MQB1R01, so there should exist no difference in the trajectories for the four different test runs. Differences were taken of the extreme values of the four measurements, and this value was compared to the average value of all four tests. In all cases, the spread of the quadrupole strengths was less than $5 \%$ of the average value for all four test runs, and in fact were under $1.5 \%$ for all but one case. Additionally, all values of $\sigma$ were within a reasonable range.

This test was successful in both localizing and resolving a known optics change to within $1 \%$ of the value indicated by the machine archive. However, if this procedure was only able to resolve this change at the $5 \%$ level, it would still be useful for the Operations crew. This is due to the nature of machine tuning. Once a problem is found with the optics of the machine, the crew will continue to make corrections to the optics until such a time that it is deemed insignificant. Should they only be able to localize the change in optics to a region between several BPMs, they will troubleshoot the suspicious area until the exact cause is found. Once the cause of the problem is located, they will make corrections as necessary. If this procedure reports that the quadrupole strength is $15 \%$ different than expected, they will make an initial correction of $15 \%$. However, they will not simply trust that the problem is gone and that the optics are correct. They will investigate further and find that the quadrupole value must be changed some small amount more in order for the optics to behave as desired.

This resolution test demonstrates that the rayTrace procedure is capable of characterizing the beamline. The information gathered through this procedure can be used by the Operations crew to not only characterize sections of the beamline, but also for tuning the machine. 


\section{Part IV}

\section{Summary and Conclusion}




\section{Chapter 7}

\section{Conclusions}

\subsection{Summary}

Characterizing the beamline of an open-ended machine such as CEBAF at Jefferson Lab poses a challenge that does not exist at circular machines. Tools already exist that can help in this process, but they lack in several key areas. As CEBAF completes its upgrade to $12 \mathrm{GeV}$, control of the beam parameters must be improved, as tolerance for errors that are currently ignored will decrease. The rayTrace procedure is an attempt to address the weaknesses of the current techniques, further supplementing the tools used by Operations to meet the beam specifications of the experimental physics users.

The procedure is as follows:

1. Set up the machine for tune-mode $(5-10 \mu A)$ beam, ensuring all relevant orbit and energy locks are off.

2. Acquire the model Twiss parameters from the Model Server.

3. On the rayTrace GUI, select the desired plane, launchpoint, optical parameters, number of data points, and number of times around the phase ellipse.

4. Run the rayTrace data collection program.

5. Using the raw data, use fitphase to calculate the angular information and Twiss parameters. This information can be used for qualitative characterization, though the level of noise in the BPM system may limit use for quantitative measurements.

6. Perform singular value decomposition on the detrended raw data in order to reduce the uncorrelated contributions to the signal. This smoothed data will then be used for quantitative analysis.

7. Use the smooth data as an input to fitphase. Recalculate the angular information and Twiss parameters with this data. 
8. Use the results of Step 7 for any analysis.

A resolution test was performed as a demonstration of this procedure's utility. This resolution tested the procedure's ability to localize and resolve a known optics change. The optics change was made by varying the strength of a single quadrupole by $\pm 20 \%$ from a Baseline value which was measured both before and after the quadrupole changes. A quadrupole was chosen near the end of Arc 1, and the launchpoint for the rayTrace procedure was chosen near the beginning of the same Arc. Data was collected through Arc 1 and Linac 2 (South Linac). This data was then smoothed using SVD, and analyzed using fitphase. The location of the optics change was found by overlaying plots of $\beta$ for the four different test runs and seeing where the runs corresponding to the changed optics deviate from the Baseline values. A fitting routine in Mad-X was used to calculate the values of the quadrupole that was changed. This routine was able to resolve the changes made to the strength of the quadrupole, calculating more than a $19 \%$ change in both the Positive and Negative tests, and reproducing the Baseline value within $1 \%$.

This resolution test was successful in meeting its two primary goals of localizing and resolving a known optics change. Furthermore, it demonstrates the ability of the rayTrace procedure to be used for characterizing and tuning CEBAF's beamline. This procedure could be used for characterizing other open-ended beamlines as well.

\subsection{Suggestions for Future Work}

As the $12 \mathrm{GeV}$ Upgrade concludes, the rayTrace procedure should prove to be a useful tool for both the commissioning process and general operations during the $12 \mathrm{GeV}$ era. There are many ways in which this procedure can be improved and expanded upon. This section will briefly discuss a few of these.

One very obvious improvement would be to streamline the process so that information can be used more rapidly. One way to do this would be to automate much of the process. If the data acquisition procedure fed the data directly into a program that could detrend it and perform SVD, allowing the user to choose what cuts are needed, and then feeding this smoothed data into a fitphasebased program, the turnaround on feedback to the Operations crew would be much more rapid. This automated procedure should store all data at every step, and also allow for the manual analysis. In many cases though, an operator could use the automated system very quickly for troubleshooting the beamline.

An extension of this procedure would be to go beyond linear optics and investigate rayTrace's use for nonlinear optics, as well as the low levels of crossplane coupling that exist in parts of the CEBAF beamline. Although $X$ - and $Y$-Plane data is gathered separately for $X$ and $Y$ kicks, respectively, cross-plane data is acquired for each plane. In general, the cross-plane signal is on the same order as the BPM noise, but it could be worthwhile to investigate methods to improve on this. 


\section{Part V}

\section{Appendices}




\section{Appendix A}

\section{Additional Background Development}

\section{A.1 Some Basic Physics}

In order to understand the symplectic nature of beam physics, the classical mechanics formulations will be discussed briefly in this section. The discussion will begin with the Lagrangian formulation. From there, the Hamiltonian formulation will be developed.

\section{A.1.1 Lagrangian Formulation}

This section will briefly discuss the Lagrangian formulation of classical mechanics. For a more complete discussion, the reader is urged to consult any text on classical mechanics, such as Goldstein or Laundau and Lifshitz [10, 14].

To begin this formulation, start with Hamilton's variational principle, which states that the integral

$$
J=\int_{t_{0}}^{t_{1}} L d t,
$$

when taken along a possible path of motion between $t_{0}$ and $t_{1}$, is an extremum when evaluated along the true path. A simpler way of stating this is, out of all of the possible ways that the system's configuration could change in the given time interval, the true motion which occurs will either maximize or minimize the action of Equation A.1. One could also describe this statement as

$$
\delta J=\delta \int_{t_{0}}^{t_{1}} L d t=0,
$$

where in both A.1 and A.2, $L \equiv L\left(q_{i}, \dot{q}_{i}, t\right)$ is the Lagrangian of the system. 
Given the constraint $\delta q_{i}=0$ at $t_{0}$ and $t_{1}$, equation A.2 evolves as:

$$
\begin{aligned}
\delta \int_{t_{0}}^{t_{1}} L d t & =0 \\
& =\int \sum_{i} \frac{\partial L}{\partial q_{i}} \delta q_{i} d t+\int \sum_{i} \frac{\partial L}{\partial \dot{q}_{i}} \delta \dot{q}_{i} d t \\
\int \frac{\partial L}{\partial \dot{q}_{i}} \delta \dot{q}_{i} d t & =\int \frac{\partial L}{\partial \dot{q}_{i}} \frac{d}{d t} \delta q_{i} d t \\
& =\left.\frac{\partial L}{\partial \dot{q}_{i}} \frac{d}{d t} \delta q_{i}\right|_{t_{0}} ^{t_{1}}-\int \frac{d}{d t} \frac{\partial L}{\partial \dot{q}_{i}} \delta q_{i} d t
\end{aligned}
$$

Due to fixed endpoints, the first term on the right is zero, so

$$
\begin{aligned}
\delta \int_{t_{0}}^{t_{1}} L d t & =\int \sum_{i}\left(\frac{\partial L}{\partial q_{i}}-\frac{d}{d t} \frac{\partial L}{\partial \dot{q}_{i}}\right) \delta q_{i} d t \\
& =0 .
\end{aligned}
$$

Assuming that the variations, $\delta q_{i}$, are independent, the above statement can only be true if its coefficients separately vanish:

$$
\frac{d}{d t} \frac{\partial L}{\partial \dot{q}_{i}}-\frac{\partial L}{\partial q_{i}}=0 .
$$

Equation A.3 is a representation of the Euler-Lagrange equation.

A simple way to describe the nature of the Euler-Lagrange equations is by considering the case of a falling mass, $m$. Setting $L=T-V=\frac{1}{2} m v^{2}-m g x$, one can simply solve A.3, getting the solution $m \dot{v}-m g=0$. Furthermore, the canonical momentum of the system can be defined as

$$
P_{i}=\frac{\partial L}{\partial \dot{q}_{i}} .
$$

One important factor in the formulation of the Lagrangian is that it should be independent of any specific frame of reference, and therefore a Lorentz invariant. A simple way to accomplish this is to use 4 -vectors to describe the system. For example, to find the Lagrangian for a particle at rest, as observed from a laboratory system that is moving in relation to the particle, one can start with 
the momentum-energy and differential space-time 4-vectors. Here,

$$
\begin{aligned}
\left(c p_{x}, c p_{y}, c p_{z}, i E\right) & =\left(0,0,0, i m c^{2}\right) \\
\frac{1}{c}(d x, d y, d z, i c d \tau)\left(c p_{x}, c p_{y}, c p_{z}, i E\right) & =-m c^{2} d \tau \\
d \tau & =\frac{1}{\gamma} d t \\
\gamma & =\sqrt{1-\beta^{2}} \\
\beta & =\frac{v}{c} \\
L & =-m c^{2} \sqrt{1-\beta^{2}} .
\end{aligned}
$$

For a charged particle in an electromagnetic (EM) field, the interaction depends upon the charge, $e$, the velocity, $v$, and the field, $A$. Noting that $\vec{A}$ is the vector potential and $\phi$ is the scalar potential, multiplication of the field and velocity 4 -vectors yields the Lagrangian as follows:

$$
\begin{aligned}
e\left(A_{x}, A_{y}, A_{z}, i \phi\right) \gamma\left(v_{x}, v_{y}, v_{z}, i\right) & =e \gamma(\vec{A} \vec{v}-\phi) \\
L & =-m c^{2} \sqrt{1-\beta^{2}}+e \vec{A} \vec{v}-e \phi .
\end{aligned}
$$

\section{A.1.2 Hamiltonian Formulation}

This section will briefly develop the Hamiltonian Formulation, basing the discussion on the previous treatment of the Lagrangian.

Beginning with the Euler-Lagrange equation, A.3, where the Lagrangian of the system is (from Equation A.6) $L=-m c^{2} \sqrt{1-\beta^{2}}+e \vec{A} \vec{v}-e \phi$, one can derive the equation for the Lorentz force, which governs the motion of a charged particle in an EM field.

Several relationships must be described before one can simply plug the Lagrangian into the Euler-Lagrange equation. The relativistic terms are described in the previous section. In addition to these, one must also know that the scalar and vector potentials are related to the electric and magnetic fields as:

$$
\begin{aligned}
\vec{E} & =-\nabla \phi-\frac{\partial \vec{A}}{\partial t} \text { and } \\
\vec{B} & =\nabla \times \vec{A} .
\end{aligned}
$$

Furthermore, the relativistic kinetic momentum is given by $\vec{p}=\gamma m \vec{v}$. When one solves the Euler-Lagrange equation for the Lagrangian above, then substitutes these values and simplifies, the Lorentz force is found;

$$
\frac{d \vec{p}}{d t}=\vec{F}=e(\vec{E}+\vec{v} \times \vec{B}) .
$$

Since $\vec{p}=\gamma m \vec{v}$ and $\vec{P}=\frac{\partial L}{\partial \dot{q}_{i}}$, one also finds that $\vec{P}=\vec{p}+e \vec{A}$. Using this, the Hamiltonian for particle motion is given by

$$
H=\vec{P} \vec{v}-L=c\left[(m c)^{2}+(\vec{P}-e \vec{A})^{2}\right]^{\frac{1}{2}}+e \phi,
$$


which gives Hamilton's equations of motion,

$$
\begin{aligned}
\dot{q}_{i} & =\frac{\partial H}{\partial P_{i}} \\
\text { and } \dot{P}_{i} & =-\frac{\partial H}{\partial q_{i}} .
\end{aligned}
$$

\section{A.2 Symplecticity}

In general, the particle beam in an accelerator obeys Hamiltonian dynamics. One way to check for proper accelerator behavior is to make sure that this statement holds true. One way to do that is to check that the transfer matrix of the beam satisfies the symplectic condition, which is a Lie algebraic representation of Hamilton's equations of motion. This section will briefly describe symplecticity.

When forces are derivable from a potential not involving the velocities, the Lagrangian of a system can be decomposed into a sum of homogeneous functions as

$$
L(q, \dot{q}, t)=L_{0}(q, t)+L_{1}(q, \dot{q}, t)+L_{2}(q, \dot{q} t)
$$

where $L_{0}$ is a homogeneous function of the generalized coordinates, $L_{1}$ is a homogeneous function of the first degree in $\dot{q}$, and $L_{2}$ is a homogeneous function of the second degree in $\dot{q}$. Even with with velocity-dependent potentials, the Lagrangian for a charged particle in an electromagnetic field (Equation A.6) satisfies this condition. When the Lagrangian can be decomposed as above, the Hamiltonian can take the form

$$
\begin{aligned}
H(q, p, t) & =\dot{q}_{i} p_{i}-L(q, \dot{q}, t) \\
& =\dot{q}_{i} p_{i}-\left[L_{0}\left(q_{i}, t\right)+L_{1}\left(q_{i}, t\right) \dot{q}_{k}+L_{2}\left(q_{i}, t\right) \dot{q}_{k} \dot{q}_{m}\right]
\end{aligned}
$$

If there is no explicit time dependence in the equations for the generalized coordinates, then this simplifies as $L_{2} \dot{q}_{k} \dot{q}_{m}=T$ where $T$ is kinetic energy. It is further simplified if the forces are derivable from a conservative potential, $\mathrm{V}$, as $L_{0}=-V$. If both of these specific conditions are true, then the Hamiltonian is simply the total energy of the system, $H=T+V$.

Taking the case where

$$
L\left(q_{i}, \dot{q}_{i}, t\right)=L_{0}(q, t)+\dot{q}_{i} a_{i}(q, t)+\dot{q}_{i}^{2} T_{i}(q, t),
$$

with $a_{i}$ and $T_{i}$ both being functions of $q$ and $t$, the algebra can be further simplified. Letting the $\dot{q}_{i}$ 's form a column matrix, $\dot{\vec{q}}$, one can rewrite as

$$
L\left(q_{i}, \dot{q}_{i}, t\right)=L_{0}(q, t)+\dot{\vec{q}}^{T} \vec{a}+\frac{1}{2} \dot{\vec{q}} T \vec{T} \dot{\vec{q}}
$$

where $\vec{T}$ is a $n \times n$ matrix whose elements are general functions of $q$ and $t, \vec{a}$ is a column matrix whose elements are general functions of $q$ and $t$, and $\dot{\vec{q}}^{T}$ is the transpose of $\dot{\vec{q}}$. 
For demonstrative purposes, taking the specific case where $q_{i}=\{x, y, z\}$ and $\vec{T}$ is diagonal, one finds that

$$
\begin{aligned}
\frac{1}{2} \dot{\vec{q}}^{T} \vec{T} \dot{\vec{q}} & =\frac{1}{2}\left[\begin{array}{lll}
\dot{x} & \dot{y} & \dot{z}
\end{array}\right]\left[\begin{array}{ccc}
m & 0 & 0 \\
0 & m & 0 \\
0 & 0 & m
\end{array}\right]\left[\begin{array}{l}
\dot{x} \\
\dot{y} \\
\dot{z}
\end{array}\right] \\
& =\frac{m}{2}\left(\dot{x}^{2}+\dot{y}^{2}+\dot{z}^{2}\right), \\
\dot{\vec{q}}^{T} \vec{a} & =\left[\begin{array}{lll}
\dot{x} & \dot{y} & \dot{z}
\end{array}\right]\left[\begin{array}{l}
a_{x} \\
a_{y} \\
a_{z}
\end{array}\right] \\
& =a_{x} \dot{x}+a_{y} \dot{y}+a_{z} \dot{z} \\
& =\vec{a} \cdot \dot{\vec{r}} .
\end{aligned}
$$

This makes the Hamiltonian

$$
H=\dot{\vec{q}}^{T}(\vec{p}-\vec{a})-\frac{1}{2} \dot{\vec{q}}^{T} \vec{T} \dot{\vec{q}}-L_{0}
$$

where $\vec{T}$ is symmetric. With a symmetric $\vec{T}$, the conjugate momenta is a column matrix $\vec{p}=\vec{T} \dot{\vec{q}}+\vec{a}$. From this, one can find $\dot{\vec{q}}=\vec{T}^{-1}(\vec{p}-\vec{a})$. The inverse of $\vec{T}$ exists because kinetic energy has a positive definite property. This also defines the transpose of $\dot{\vec{q}}, \dot{\vec{q}}^{T}=\left(\vec{p}^{T}-\vec{a}^{T}\right) \vec{T}^{-1}$. Plugging into the definition of the Hamiltonian, the functional form becomes

$$
H(q, p, t)=\frac{1}{2}\left(\vec{p}^{T}-\vec{a}^{T}\right) \vec{T}^{-1}(\vec{p}-\vec{a})-L_{0}(q, t) .
$$

For an accelerator, one must investigate the dynamics of a particle beam in an electromagnetic field. This can be done in the way described above. The following will look at the simplified case of a single, nonrelativistic particle in an electromagnetic field, where the Lagrangian is a simplified version of Equation A.6:

$$
L=\frac{1}{2} m \vec{v}^{2}-q \phi+q \vec{A} \cdot \vec{v}
$$

where the scalar potential, $-q \phi$, is the $L_{0}$ term of the Lagrangian, and the vector potential, $q \vec{A} \cdot \vec{v}$, is the $L_{1}$ term. Choosing Cartesian coordinates, the Lagrangian is

$$
L=\frac{m}{2} \dot{x}_{i} \dot{x}_{i}+q A_{i} \dot{x}_{i}-q \phi
$$

where the potentials are functions of $x_{i}$ and time. This gives a linear term in generalized velocities, meaning matrix $\vec{a}$ has elements $q A_{i}$. The Hamiltonian will not simply be $H=T+V$ because of this linear term, however, it will still be the total energy of the system because the potential energy in the electromagnetic field is determined by $\phi$ alone. The canonical momenta will be $p_{i}=m \dot{x}_{i}+q A_{i}$. The Hamiltonian can then be written

$$
H=\frac{1}{2 m}\left(p_{i}-q A_{i}\right)\left(p_{i}-q A_{i}\right)+q \phi=E .
$$


Forming $p_{i}$ into a vector, $\vec{p}$

$$
H=\frac{1}{2 m}(\vec{p}-q \vec{A})^{2}+q \phi
$$

For a system with $n$ degrees of freedom, construct a column matrix $\vec{\eta}$ with $2 n$ elements, where $\eta_{i}=q_{i}$ and $\eta_{i+n}=p_{i}$, where $i \leq n$. Next construct a column matrix $\frac{\partial H}{\partial \vec{\eta}}$ which has the elements $\left(\frac{\partial H}{\partial \vec{\eta}}\right)_{i}=\frac{\partial H}{\partial q_{i}}$ and $\left(\frac{\partial H}{\partial \vec{\eta}}\right)_{i+n}=\frac{\partial H}{\partial p_{i}}$. Next, define $\vec{J}$ as a $2 n \times 2 n$ matrix composed of $4 n \times n$ zero and unit matrices:

$$
\vec{J}=\left[\begin{array}{cc}
\overrightarrow{0} & \overrightarrow{1} \\
-1 & \overrightarrow{0}
\end{array}\right]
$$

where

$$
\overrightarrow{0}=\left[\begin{array}{ll}
0 & 0 \\
0 & 0
\end{array}\right]
$$

and

$$
\overrightarrow{1}=\left[\begin{array}{ll}
1 & 0 \\
0 & 1
\end{array}\right]
$$

The transpose of $\vec{J}$ is equal to its inverse, and its determinant is unity. With these definitions, Hamilton's equations of motion can be written in what is called symplectic notation:

$$
\vec{\eta}=\vec{J} \frac{\partial H}{\partial \vec{\eta}}
$$

Expanded for a two-coordinate system, this would look like

$$
\left[\begin{array}{c}
\dot{q}_{1} \\
\dot{q}_{2} \\
\dot{p}_{1} \\
\dot{p}_{2}
\end{array}\right]=\left[\begin{array}{cccc}
0 & 0 & 1 & 0 \\
0 & 0 & 0 & 1 \\
-1 & 0 & 0 & 0 \\
0 & -1 & 0 & 0
\end{array}\right]\left[\begin{array}{c}
-\dot{p}_{1} \\
-\dot{p}_{2} \\
\dot{q}_{1} \\
\dot{q}_{2}
\end{array}\right]
$$




\section{Appendix B}

\section{Scripts}

\section{B.1 Example Lattice File and Mad-X Script}

\section{B.1.1 Lattice File}

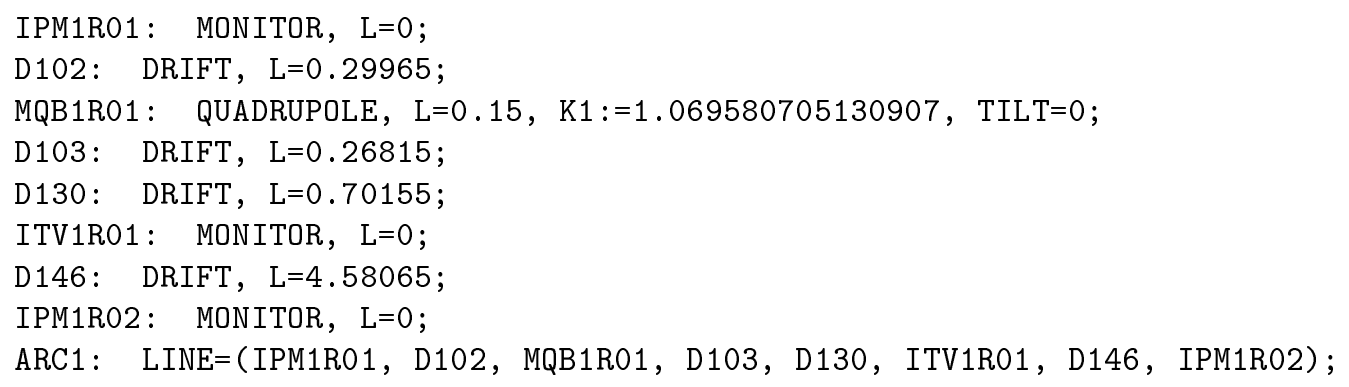




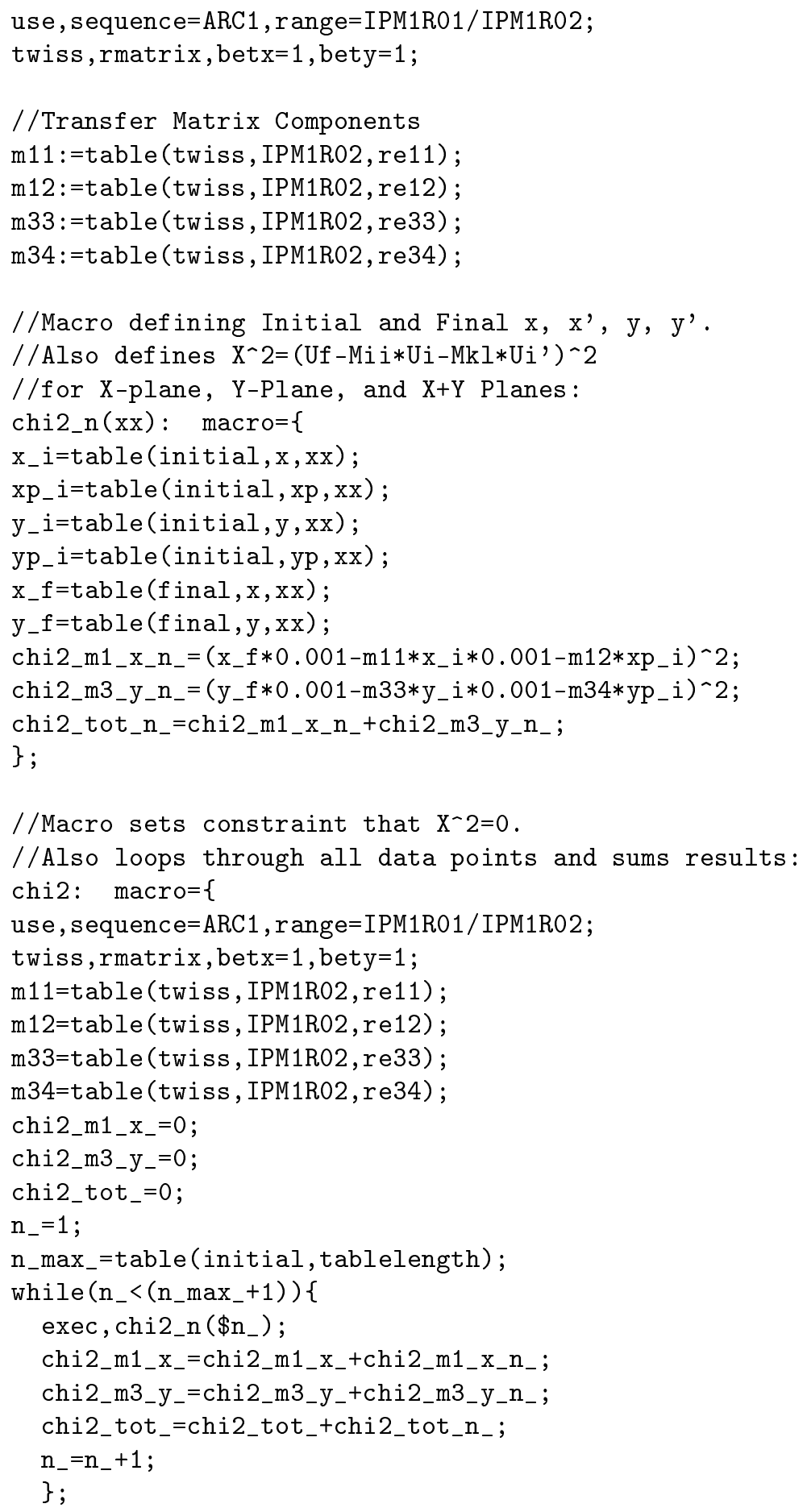




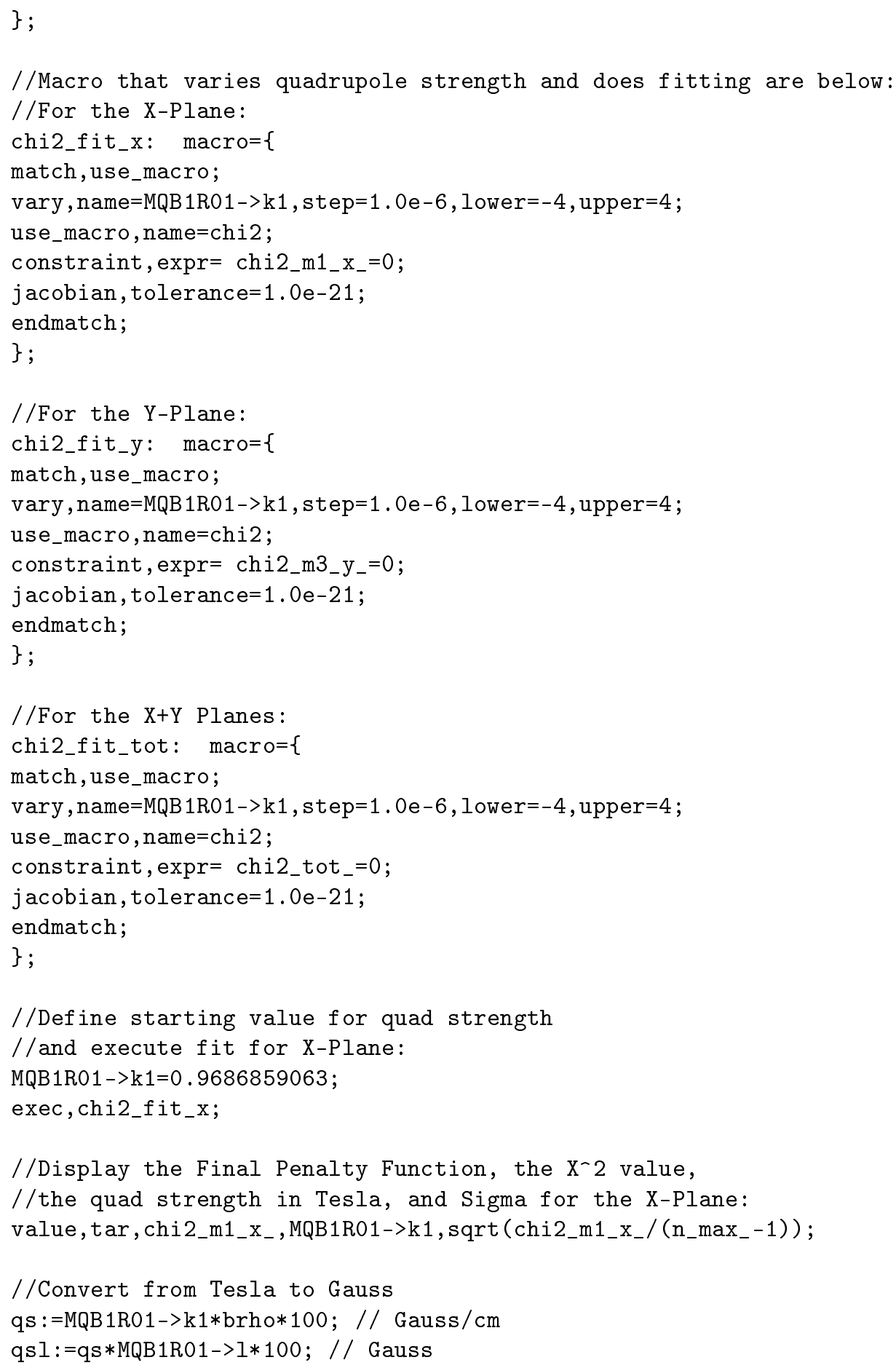




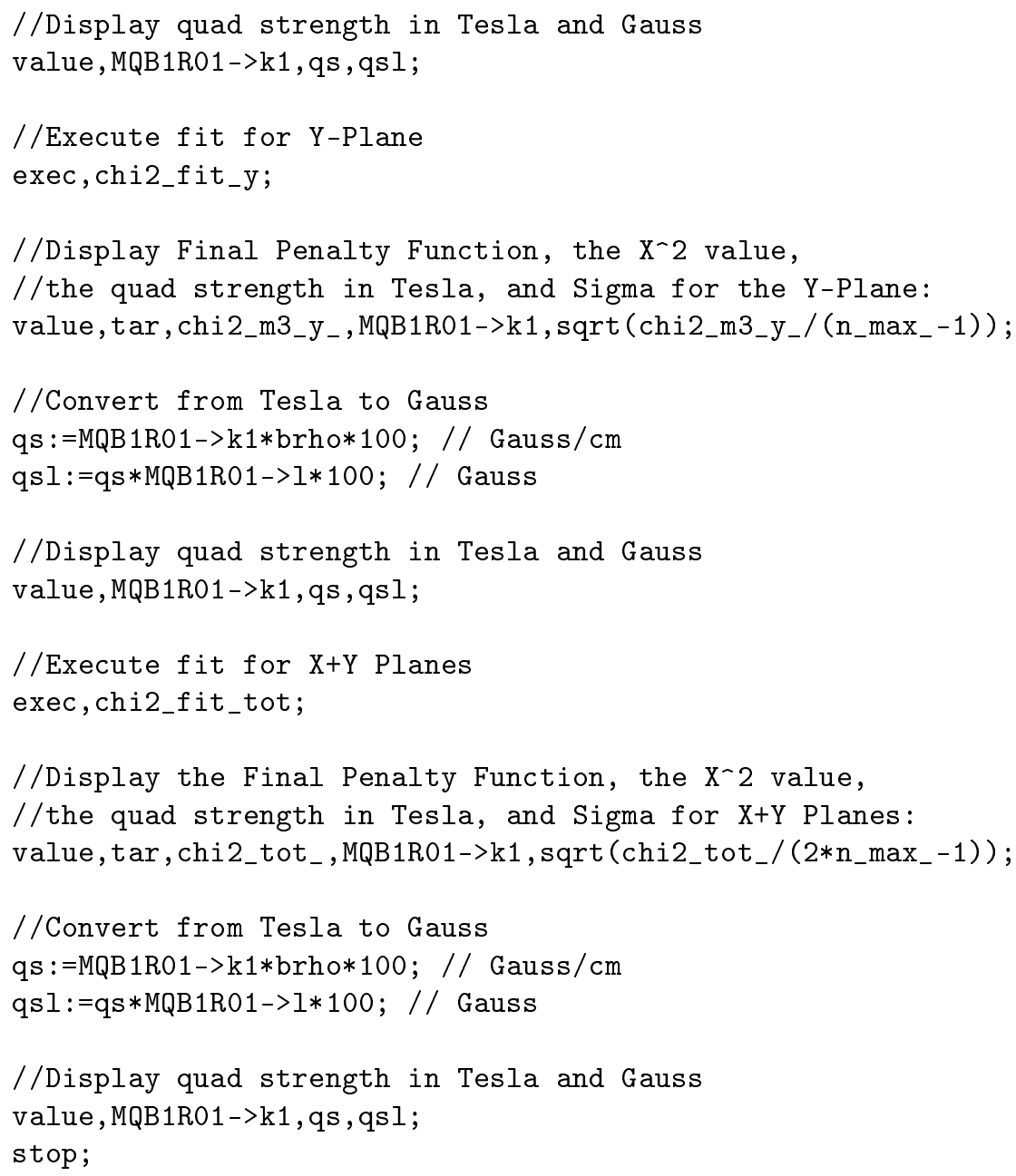




\section{Bibliography}

[1] Bethany Adams and Nina Magnoni. Singular value decomposition: Compression of color images. Shows basics of how to use SVD.

[2] C.E Adolphsen, T.L. Lavine, W.B. Atwood, T.M. Himel, M.J. Lee, T.S. Mattison, R. Pitthan, J.T. Seeman, S.H. Williams, and G.H. Trilling. Beam-based alignment technique for the slc linac. Technical report, Stanford Linear Accelerator AND Lawrence Berkeley Laboratory, 1989.

[3] Timotthy L. Barklow, Yu-Chiu Chao, Andrew Hutton, Nobukazo Toge, and Nicholas J. Walker. Commissioning experience with the slc arcs. In Presented at the XIV International Conference on High Energy Accelerators, Tsukuba, Japan, August 22-26, 1989, 1989.

[4] Martin Berz. Advances in Imaging and Electron Physics: Modern Map Methods in Particle Beam Physics Volume 108. Academic Press, 1999.

[5] K.L. Brown and R.V. Servranckx. Cross-plane coupling and its effect on projected emittance. University, 1989.

[6] A. Chao. Handbook of Accelerator Physics and Engineering. World Scientific, 1999.

[7] Ernest D. Courant, M. Stanley Livingston, and Hartland S. Snyder. The strong-focusing synchroton - a new high energy accelerator. Phys. Rev., 88:1190-1196, Dec 1952.

[8] David Douglas, Etienne Forest, and Roger V. Servranckx. A Method to Render Second-Order Beam Optics Programs Symplectic. IEEE Trans.Nucl.Sci., 32:2279-2281, 1985.

[9] Peter Forck, Piotr Kowina, and Dmitry Liakin. Beam Position Monitors. 2008. Gesellschaft fšur Schwerionenforschung GSI, Darmstadt, Germany.

[10] H. Goldstein. Classical Mechanics. Addison-Wesley world student series. Addison-Wesley Press, third edition, 2002.

[11] D.J. Griffiths. Introduction to electrodynamics. Prentice Hall, 1999. 
[12] A.S. Hofler, B.A. Bowling, C.S. Higgins, P.K. Kloeppel, G.A. Krafft, and K.L. Mahoney. Performance of the cebaf arc beam position monitors. In PAC 1993 Proceedings, pages 2298-2300, 1993.

[13] J.D. Jackson. Classical Electrodynamics. John Wiley \& Sons, 1998.

[14] L.D. Landau and E.M.A. Lifshitz. Course of Theoretical Physics: Mechanics. Course of Theorical Physics. Pergamon Press, 1976.

[15] S.Y. Lee. Accelerator Physics. World Scientific, 2004.

[16] Christoph W. Leemann, David R. Douglas, and Geoffrey A. Krafft. The continuous electron beam accelerator facility: Cebaf at the jefferson laboratory. Annual Review of Nuclear and Particle Science, 51:413-50, 2001.

[17] B.E. Norum, J.S. McCarthy, and R.C. York. Cebaf - a high energy, high duty factor electron accelerator for nuclear physics. Nuclear Instruments and Methods in Physics Research, B10/11:337-341, 1985.

[18] Jon Shlens. A tutorial on principal component analysis: Derivation, discussion and singular value decomposition, version 1. 2003.

[19] Michael Spata. Application of Chebyshev Formalism to Identify Nonlinear Magnetic Field Components in Beam Transport Systems. PhD thesis, Old Dominion University, 2012.

[20] P. Tenenbaum and T. O. Raubenheimer. Resolution and systematic limitations in beam-based alignment. Phys. Rev. Special Topics - Accelerators and Beams, 3:052801-1-12, 2000.

[21] Various. The Development of Colliders. AIP Press, 1995.

[22] N Walker, Timothy L Barklow, P Emma, and P Krejcik. Correction of the first order beam transport of the slc arcs. (SLAC-PUB-5560):3 p, May 1991.

[23] C Wang and J C Irwin. Model Independent Analysis Of Beam Centroid Dynamics In Accelerators. PhD thesis, Stanford Univ., Stanford, CA, 2000.

[24] Catherine Westfall. The founding of cebaf, 1979 to 1987, September 1994.

[25] H. Wiedemann. Particle Accelerator Physics: Part I - Basic Principles and Linear Beam Dynamics. Physics and astronomy online library. Springer, 2003.

[26] H. Wiedemann. Particle Accelerator Physics: Part II - Nonlinear and Higher-Order Beam Dynamics. Particle Accelerator Physics. Springer, 2003.

[27] H. Wiedemann. Particle Accelerator Physics. Springer, 2007. 
[28] Chun xi Wang, John Irwin, Karl Bane, Yunhai Cai, Franz J. Decker, Michiko Minty, and Yiton T. Yan. Model independent analysis of beam dynamics in accelerators. Stanford Linear Accelerator Center, Stanford University, Stanford, CA 94309.

[29] Hongguo Xu. An svd-like matrix decomposition and its applications. Department of Mathematics, University of Kansas, Lawrence, KS 66045, USA.

[30] F. Zimmermann. Measurement and correction of accelerator optics. In Joint US-CERN-Japan-Russian School on Beam Measurement, Montreux, Switzerland, 1998. 